\title{
Inequalities of General Convex Functions and Applications
}

\author{
Milan R. TASKOVIĆ
}

\begin{abstract}
This paper presents very characteristic illustrations of transversal sets (as upper, lower and middle) via general convex functions. Since the general convex functions are defined by a functional inequality, it is not surprising that this notation will lead to a number od important inequalities. This fact is connected de facto with the notation of transversal sets.
\end{abstract}

\section{General J-Convex functions}

In this section we continue the study of the general J-convex functions, which are introduced in the former paper by Task ovi ć: Mat. Japonica, 37 (1992), 367-372. We prove that if $D \subset \mathbb{R}^{n}$ a convex and open set, and if $f: D \rightarrow \mathbb{R}$ is a general J-inner function with the property of local oscillation in $D$, then it is continuous in $D$.

Since every J-convex function (also an additive function) is general J-inner function, we obtain as a particular case of the preceding statement the result of $\mathrm{F}$. B e r n s t e i n and G. D o e t s ch.

1.1. Introduction and history. Let $D \subset \mathbb{R}^{n}$ be a convex and open set. A function $f: D \rightarrow \mathbb{R}$ is called $\mathbf{J}$-convex (or Jensen convex) if it satisfies Jensen's functional inequality

$$
f\left(\frac{x+y}{2}\right) \leq \frac{f(x)+f(y)}{2}
$$

for all $x, y \in D$. If the inequality in $(\mathrm{J})$ for $x \neq y$ is sharp, $f$ is called strictly J-convex. These functions were introduced (for $n=1$ ) by J. W. Jensen in 1906, although functions satisfying similar conditions were already treated by Hadamard, Hermite, Hölder and Stolz. ${ }^{1}$

\footnotetext{
${ }^{1}$ Historical facts. The recognition of J-convex functions as a class of functions to be studied is generally traced to $\mathrm{J}$ e $\mathrm{n} \mathrm{se} \mathrm{n}$, but as is usually the case, earlier work can be cited that anticipated what was to come. Hölder proved that if $f^{\prime \prime}(x)>0$, then $f$ satisfied what later came to be known as Jensen's inequality. S tolz proved that if $f$ is continuous on $[a, b]$ and satisfies: $(\mathrm{J})$, then $f$ has left and right derivatives at each point of $(a, b)$. H a d a m a r d obtained a basic integral inequality for functions having
} 
Basic properties of J-convex functions in the one-dimensional case were proved by Jensen himself and by Bernstein-Doetsch in 1915. Generalizatios to higher dimensions were made by Blumberg and Mohr.

Fréchet in 1913 has proved that a measurable function which satisfies the following Cauchy's functional equation of addivity

$$
f(x+y)=f(x)+f(y)
$$

is continuous. Sierpiński in 1920 has proved that a measurable J-convex function is also continuous. ${ }^{2}$

Various other proofs of the preceding statements were then supplied by Banach, Kac, Alexiewicz-Orlicz, Kuczma-Smital, Paganoni, Fisher-Slodowski, Figiel, S. Kurepa, Seneta and Steinhaus.

Ostrowski in 1923 has proved the statement according to which a J-convex function bounded on a set of the positive measure is continuous.

In the present section we prove some analogous statements of the preceding type for general J-convex (inner) functions.

In our former paper, Tasković in 1992, has introduced the notion of general J-convex functions. A function $f: D \rightarrow \mathbb{R}$, where $\mathbb{R}$ denotes the real line and $D$ is a convex subset of $\mathbb{R}^{n}$, is said to be general $\mathbf{J}$-convex if there is a function $g: f(D)^{2} \rightarrow \mathbb{R}$ such that

$$
f\left(\frac{x+y}{2}\right) \leq \max \{f(x), f(y), g(f(x), f(y))\}
$$

for all $x, y \in D$. We notice that the set of all J-convex functions can be a proper subset of the set of all general J-convex functions.

On the other hand, recall that a function $f: D \rightarrow \mathbb{R}$ is said to be general J-concave if there is a function $d: f(D)^{2} \rightarrow \mathbb{R}$ such that

$$
\min \{f(x), f(y), d(f(x), f(y))\} \leq f\left(\frac{x+y}{2}\right)
$$

for all $x, y \in D$. If $f: D \rightarrow \mathbb{R}$ is general J-convex and a general J-concave function, then $f$ is a general $\mathbf{J}$-inner function.

an increasing derivative on $[a, b]$ in the following form as

$$
f\left(\frac{a+b}{2}\right) \leq \frac{1}{b-a} \int_{a}^{b} f(x) d x \leq \frac{f(a)+f(b)}{2} ;
$$

among the rest $\mathrm{J}$ e $\mathrm{n}$ s e $\mathrm{n}$ used $(\mathrm{J})$ to define convex functions and gave the first in a long series of results which together with $(\mathrm{J})$ imply the continuity of $f$.

${ }^{2}$ Additive functions. A function $f: \mathbb{R}^{n} \rightarrow \mathbb{R}$ (for a fixed $n \in \mathbb{N}$ ) is called additive iff it satisfies Cauchy's functional equation in the form (Ca) for all $x, y \in \mathbb{R}^{n}$. For $n=1$ equation (Ca) was first treated in 1791 by A. M. L e g e n d r e and in 1809 C. F. G a u s s, but A.L. C a u c hy in 1821 first found its general continuous solution, and the equation has been named after him. Additional functions with infinite values were considered by $\mathrm{H}$ a l p e r i n [1948]. Concerning equation ( $\mathrm{Ca}$ ) and the vast literature of the subject, cf. also A c z él [1966]. 
We notice that the set of all J-convex and J-concave functions can be a proper subset of the set of all general J-inner functions.

1.2. Local boundedness of general J-inner functions. Let $D \subset \mathbb{R}^{n}$. A function $f: D \rightarrow \mathbb{R}$ is called locally bounded (locally bounded above, locally bounded below) at a point $x_{0} \in D$ if there exists a neighborhood $U \subset D$ of $x_{0}$ such that the function $f$ is bounded (bounded above, bounded below) on $U$. We start with the following statements from Tasković in 1995.

Lemma 1. Let $D \subset \mathbb{R}^{n}$ be a convex and open set, and let $f: D \rightarrow \mathbb{R}$ be a general J-convex function for some bounded above function $g: f(D)^{2} \rightarrow \mathbb{R}$. If $f$ is locally bounded above at a point $x_{0} \in D$, then it is locally bounded above at every point $x \in D$.

A brief proof of this statement may be found at Tasković in 1995. We notice that when considered dually and analogously to the preceding Lemma 1 , we obtain directly the following statement.

Lemma 2. (Dually of Lemma 1). Let $D \subset \mathbb{R}^{n}$ be a convex and open set, and let $f: D \rightarrow \mathbb{R}$ be a general J-concave function for some bounded below function $g: f(D)^{2} \rightarrow \mathbb{R}$. If $f$ is locally bounded below at a point $x_{0} \in D$, then it is locally bounded below at every point $x \in D$.

Thus, when we combine two preceding lemmas, we directly obtain the following statement for general J-inner functions.

Theorem 1. Let $D \subset \mathbb{R}^{n}$ be a convex and open set, and let $f: D \rightarrow \mathbb{R}$ be a general J-inner function for some bounded function $g: f(D)^{2} \rightarrow \mathbb{R}$. If $f$ is locally bounded at a point $x_{0} \in D$, then it is locally bounded at every point $x \in D$.

A brief proof of this statement may be found at Tasković in 1995. Since Jconvex functions have support lines, thus, we notice that J-convex functions are, de facto, general J-inner functions. Thus we obtain directly the following statement.

Corollary 1. Let $D \subset \mathbb{R}^{n}$ be a convex and open set, and let $f: D \rightarrow \mathbb{R}$ be a Jconvex function. If $f$ is locally bounded above at a point $x_{0} \in D$, then it is locally bounded at every point $x \in D$.

1.3. Inequalities of general $\mathbf{J}$-convexity. We begin with the following essential statements.

Theorem 2. Let $D \subset \mathbb{R}^{n}$ be a convex and open set. If $f: D \rightarrow \mathbb{R}$ is a general J-convex function, then there is a function $g: f(D)^{n} \rightarrow \mathbb{R}$ such that (Da) $f\left(\frac{x_{1}+\cdots+x_{n}}{n}\right) \leq \max \left\{f\left(x_{1}\right), \ldots, f\left(x_{n}\right), g\left(f\left(x_{1}\right), \ldots, f\left(x_{n}\right)\right)\right\}$ for every $n \in \mathbb{N}$ and for every $x_{1}, \ldots, x_{n} \in D$. Equality holds in (Da) if and only if $x:=x_{1}=\cdots=x_{n}$ and $f(x)=g(f(x), \ldots, f(x))$. 
Induction shows that this statement holds. As an immediate consequence of Theorem 2 we obtain the following statement.

Theorem 2a. Let $D \subset\left\{\mathbb{R}^{n}\right.$ be a convex and open set. If $f: D \rightarrow \mathbb{R}$ is a general J-convex function, then there is a function $g: f(D)^{2} \rightarrow \mathbb{R}$ such that.

$$
f(\lambda x+(1-\lambda) y) \leq \max \{f(x), f(y), g(f(x), f(y))\}
$$

for every $x, y \in D$ and for every $\lambda \in \mathbb{Q} \cap[0,1]$, where $\mathbb{Q}$ denotes the set of rational numbers.

We notice, if $f: D \rightarrow \mathbb{R}$ is general J-convex and continuous, then (Q) holds for all real $\lambda \in[0,1]$.

Next, we give an interesting inequality for general J-convex functions which is, in some sense, the best of its kind.

Theorem 3. Let $D \subset \mathbb{R}^{n}$ be a convex set and let $F \subset \mathbb{R}$ be a field. If a function $f: D \rightarrow \mathbb{R} \cup\{-\infty\}$ satisfies (Q) for every $\lambda \in F \cap[0,1]$, then

$$
f\left(\sum_{k=1}^{n} \lambda_{k} x_{k}\right) \leq \max \left\{f\left(x_{1}\right), \ldots, f\left(x_{n}\right), g\left(f\left(x_{1}\right), \ldots, f\left(x_{n}\right)\right)\right\}
$$

for some function $g: f(D)^{n} \rightarrow \mathbb{R}$, for every $n \in \mathbb{N}$, for arbitrary $\lambda_{1}, \ldots, \lambda_{n} \in$ $F \cap[0,1]$ such that $\lambda_{1}+\cdots+\lambda_{n}=1$ and for all $x_{1}, \ldots, x_{n} \in D$.

Thus inequality (DN) with arbitrary $x_{1}, \ldots, x_{n} \in D$ is valid for every general J-convex function $f: D \rightarrow \mathbb{R}$ with arbitrary $\lambda_{1}, \ldots, \lambda_{n} \in \mathbb{Q} \cap[0,1]$, adding up to 1 , and for every continuous general J-convex function $f: D \rightarrow$ $\mathbb{R}$ with arbitrary $\lambda_{1}, \ldots, \lambda_{n} \in[0,1]$ adding up to 1 .

In connection with the preceding facts and Theorem 3 we have the following statement as an analytic phenomenon.

Theorem 4. (Necessity of inequality $(\mathrm{DN})$ ). Let $D \cup\{0\} \subset \mathbb{R}^{n}$ be a convex set and let $F \subset \mathbb{R}$ be a field. If $f: D \cup\{0\} \rightarrow \mathbb{R} \cup\{-\infty\}$ for some $2 \leq \rho \leq n$ and for all $\lambda_{1}, \ldots, \lambda_{\rho} \in F \cap[0,1]$ with the properties $\lambda_{1}+\cdots+\lambda_{\rho}=1$ and

$$
f\left(\sum_{i=1}^{\rho} \lambda_{i} x_{i}\right) \leq \max \left\{f\left(x_{1}\right), \ldots, f\left(x_{\rho}\right), g\left(f\left(x_{1}\right), \ldots, f\left(x_{\rho}\right)\right)\right\}
$$

for a function $g: f(D \cup\{0\})^{\rho} \rightarrow \mathbb{R}$ and for all points $x_{1}, \ldots, x_{\rho} \in D$, then $f$ is a general J-convex function.

An illustration. A function $f: D \rightarrow \mathbb{R}$, where $\mathbb{R}$ denotes the real line and $D$ is a convex subset of $\mathbb{R}^{n}$, is said to be $\psi(J)$-convex function if there is a function $\psi: \mathbb{R}_{+}^{0} \rightarrow \mathbb{R}_{+}^{0}$ such that $\psi(0)=0$ and

$$
f\left(\frac{x+y}{2}\right) \leq \frac{f(x)+f(y)}{2}+\frac{1}{2} \psi(\|x-y\|)
$$

for all points $x, y \in D$. Next, we give an inequality for this special subclass of the class of general J-convex functions. 
Proposition 1 (Tasković, [2001, p. 331]). Let $D \subset \mathbb{R}^{n}$ be a convex and open set. If $f: D \rightarrow \mathbb{R}$ is a $\psi(J)$-convex function, then the following inequality holds in the form as

$$
f\left(\frac{x_{1}+\cdots+x_{n}}{n}\right) \leq \frac{1}{n} \sum_{i=1}^{n} f\left(x_{i}\right)+\frac{1}{n} \sum_{i<j \leq n} \psi\left(\left\|x_{i}-x_{j}\right\|\right),
$$

for every $n \in \mathbb{N}$ and for all points $x_{1}, \ldots, x_{n} \in D$. Equality holds in $(T)$ if and only if $x_{1}=x_{2}=\cdots=x_{n}$.

In connection with the preceding, a function $f: D \rightarrow \mathbb{R}$, where $D$ is a convex subset of $\mathbb{R}^{n}$, is said to be general $\psi(J)$-convex function if there exist a function $g: f(D)^{2} \rightarrow \mathbb{R}$ and an increasing function $\psi: \mathbb{R}_{+}^{0} \rightarrow \mathbb{R}_{+}^{0}$ such that $\psi(0)=0$ and

$$
f\left(\frac{x+y}{2}\right) \leq \max \{f(x), f(y), g(f(x), f(y))\}+\psi(\|x-y\|)
$$

for all points $x, y \in D$. Next, we give an inequality for this special subclass of the class of general J-convex functions.

Proposition 2 (Tasković, [2001, p. 333]). Let $D \subset \mathbb{R}^{n}$ be a convex and open set. If $f: D \rightarrow \mathbb{R}$ is a general $\psi(J)$-convex function, then there is a function $g: f(D)^{n} \rightarrow \mathbb{R}$ such that

$$
\begin{aligned}
f\left(\frac{x_{1}+\cdots+x_{n}}{n}\right) & \leq \max \left\{f\left(x_{1}\right), \ldots, f\left(x_{n}\right), g\left(f\left(x_{1}\right), \ldots, f\left(x_{n}\right)\right)\right\}+ \\
+ & \frac{1}{n} \sum_{i=1}^{n} \psi\left(\left\|x_{i}-\frac{1}{n} \sum_{i=1}^{n} x_{i}\right\|\right)
\end{aligned}
$$

for every $n \in \mathbb{N}$ and for all points $x_{1}, \ldots, x_{n} \in D$. Equality holds in (B) if and only if $x:=x_{1}=\cdots=x_{n}$ and $f(x)=g(f(x), \ldots, f(x))$.

On the other hand, for the class of general $\psi(J)$-convex functions the following statement holds, also.

Proposition 3 (Tasković, [2001]). Let $D \subset \mathbb{R}^{n}$ be a convex and open set. If $f: D \rightarrow \mathbb{R}$ is a general $\psi(J)$-convex function, then there exist a function $g: f(D)^{2} \rightarrow \mathbb{R}$ and a function $\psi: \mathbb{R}_{+}^{0} \rightarrow \mathbb{R}_{+}^{0}$ satisfying $\psi(0)=0$ such that

$f(f(\lambda x+(1-\lambda) y)) \leq \max \{f(x), f(y), g(f(x), f(y))\}+\max \{\lambda, 1-\lambda\} \psi(\|x-y\|)$

for every $\lambda \in \mathbb{Q} \cap[0,1]$ and for all points $x, y \in D$. Equality in $(A)$ holds if and only if $x=y$ and $f(x)=g(f(x), f(x))$.

Next, we give an interesting inequality for general $\psi(J)$-convex functions which is, in some sense, the best of its kind.

Proposition 4 (Tasković, [2001]). Let $D \subset \mathbb{R}^{n}$ be a convex set and let $F \subset \mathbb{R}$ be a field. If $f: D \rightarrow \mathbb{R} \cup\{-\infty\}$ satisfies (A) for every $\lambda \in F \cap[0,1]$, then there is a function 
$g: f(D)^{n} \rightarrow \mathbb{R}$ such that

$$
\begin{aligned}
f\left(\sum_{i=1}^{n} \lambda_{i} x_{i}\right) & \leq \max \left\{f\left(x_{1}\right), \ldots, f\left(x_{n}\right), g\left(f\left(x_{1}\right), \ldots, f\left(x_{n}\right)\right)\right\}+ \\
& +\sum_{i<j \leq n} \max \left\{\lambda_{i}, \lambda_{j}\right\} \psi\left(\left\|x_{i}-x_{j}\right\|\right)
\end{aligned}
$$

for every $n \in \mathbb{N}$, for all points $x_{1}, \ldots, x_{n} \in D$ and for arbitrary numbers $\lambda_{1}, \ldots, \lambda_{n} \in$ $F \cap[0,1]$ such that $\lambda_{1}+\cdots+\lambda_{n}=1$.

Hence inequality $(\mathrm{R})$ with arbitrary $x_{1}, \ldots, x_{n} \in D$ is valid for every general $\psi(J)$-convex function $f: D \rightarrow \mathbb{R}$ with arbitrary $\lambda_{1}, \ldots, \lambda_{n} \in \mathbb{Q} \cap$ $[0,1]$, adding up to 1 , and for every continuous general $\psi(J)$-convex function $f: D \rightarrow \mathbb{R}$ with arbitrary $\lambda_{1}, \ldots, \lambda_{n} \in[0,1]$ adding up to 1 .

Some illustrations. We notice, G o d u n o va-L e v i n [1985] introduced the following class of functions. A function $f: D \rightarrow \mathbb{R}_{+}^{0}$, where $D$ is a convex subset of $\mathbb{R}^{n}$, is said to be GL-convex function if it satisfies inequality

$$
f(\lambda x+(1-\lambda) y) \leq \frac{f(x)}{\lambda}+\frac{f(y)}{1-\lambda}
$$

for all points $x, y \in D$ and for arbitrary $\lambda \in(0,1)$. (Clearly, nonnegative monotonic and nonnegative convex functions belong to class of GL-functions.)

In the preceding context, by $\mathrm{Polzak}$ [1966], a function $f: D \rightarrow \mathbb{R}$, where $D$ is a convex subset of $\mathbb{R}^{n}$, is said to be $\alpha(J)$-convex function if there is an $\alpha \in \mathbb{R}$ such that

$$
f\left(\frac{x+y}{2}\right) \leq \frac{f(x)+f(y)}{2}+\frac{\alpha}{4}\|x-y\|^{2}
$$

for all points $x, y \in D$. If $\alpha>0$, then $f$ is a weakly $\alpha(J)$-convex function; and if $\alpha<0$, then $f$ is a strongly $\alpha(J)$-convex function.

For further facts on $\alpha(J)$-convex functions see: $\mathrm{P}$ o l z a k [1966], L e v i t a n-P o l z a k [1966], and L y u b i ch-M a is trovski j [1970].

Proposition 5 (Tasković, [2001]). Let $D \subset \mathbb{R}^{n}$ be a convex set and let $F \subset \mathbb{R}$ be a field. If $f: D \rightarrow \mathbb{R}_{+}^{0}$ satisfies the following inequality of the form as

$$
f(\lambda x+(1-\lambda) y) \leq \frac{f(x)}{\lambda}+\frac{f(y)}{1-\lambda}
$$

for all points $x, y \in D$ adn for arbitrary $\lambda \in F \cap(0,1)$, then the following inequality holds in the form as

$$
f\left(\sum_{k=1}^{n} \lambda_{k} x_{k}\right) \leq \sum_{k=1}^{n} \frac{f\left(x_{k}\right)}{\lambda_{k}}
$$

for every number $n \in \mathbb{N} \backslash\{1\}$, for arbitrary $\lambda_{1}, \ldots, \lambda_{n} \in F \cap(0,1)$ satisfying $\lambda_{1}+\cdots+\lambda_{n}=$ 1 , and for all points $x_{1}, \ldots, x_{n} \in D$.

On the other hand, for $\psi(J)$-convex functions the following adequate statement holds, as a consequence of Proposition 4, in the form as.

Proposition $6\left(\alpha(J)\right.$-convex functions). Let $D \subset \mathbb{R}^{n}$ be a convex and open set. If $f: D \rightarrow \mathbb{R}$ is a $\alpha(J)$-convex function, then the following inequality holds in the form as

$$
f(\lambda x+(1-\lambda) y) \leq \lambda f(x)+(1-\lambda) f(y)+\alpha \lambda(1-\lambda)\|x-y\|^{2}
$$

for every number $\lambda \in \mathbb{Q} \cap[0,1]$ and for all points $x, y \in D$. Equality holds in $(\alpha)$ if and only if $x=y$. 
1.4. Continuity of general J-inner functions. A statement of BernsteinDoetsch in 1915 says that if $D \subset \mathbb{R}^{n}$ is a convex and open set, $f: D \rightarrow \mathbb{R}$ is a J-convex function, $T \subset D$ is open and nonempty, and $f$ is bounded above on $T$, then $f$ is continuous in $D$. Are there other sets $T$ for general J-convex and J-inner functions with this property? In this section we will deal with such questions.

In connection with the preceding, let $D \subset \mathbb{R}^{n}$. A general J-inner function $f: D \rightarrow \mathbb{R}$ for some function $g: f(D)^{2} \rightarrow \mathbb{R}$ is called locally oscilation at a point $x_{0} \in D$ iff there exists a neighbourhood $U \subset D$ of $x_{0}$ such that

$\max \{f(x), f(y), g(f(x), f(y))\}-\min \{f(z), f(t), g(f(z), f(t))\}<C\left\|x-x_{0}\right\|$ for some real constant $C>0$, and for all $x, y, z, t \in U$.

A mapping $f: D \rightarrow \mathbb{R}$ is with the property of local oscillation in $D$ if it have the locally oscillation at every point of $D$.

We are now in a position to formulate the following statement with who we precision, correction and expand an our former result.

Theorem 5. Let $D \subset \mathbb{R}^{n}$ be a convex and open set, and let $f: D \rightarrow \mathbb{R}$ be a general J-inner function for some bounded function $g: f(D)^{2} \rightarrow \mathbb{R}$. If $f$ with the property of local oscillation in $D$, then it is continuous in $D$.

Proof. Let $f$ be a locally oscilation at a point of $D$. Take an arbitrary $x_{0} \in D$. Thus, there exists constant $r>0$ such that $K\left(x_{0}, r\right) \subset D$ and (Os) for all $x, y, z, t \in K\left(x_{0}, r\right)$. For an arbitrary $x \in K\left(x_{0}, r\right)$ we have $\left\|x-x_{0}\right\|<r$, and consequ- ently we can find a $\lambda \in \mathbb{Q} \cap(0,1)$ such that $\left\|x-x_{0}\right\| r^{-1}<\lambda<2\left\|x-x_{0}\right\| r^{-1}$. Put

$$
y=x_{0}+\lambda^{-1}\left(x-x_{0}\right), \quad z=x_{0}-\lambda^{-1}\left(x-x_{0}\right) .
$$

Hence we have $\left\|y-x_{0}\right\|<r$, and $\left\|z-x_{0}\right\|<r$, which means that $y, z \in K\left(x_{0}, r\right)$. Also, we obtain that $x=\lambda y+(1-\lambda) x_{0}$ and $x_{0}=(1+$ $\lambda)^{-1} x+(1+\lambda)^{-1} \lambda z$. By Theorem $2 \mathrm{a}$, since $f$ is general J-inner we have from $(\mathrm{M})$ and $(\mathrm{N})$

$\min \left\{f(y), f\left(x_{0}\right), g\left(f(y), f\left(x_{0}\right)\right)\right\} \leq f(x) \leq \max \left\{f(y), f\left(x_{0}\right), g\left(f(y), f\left(x_{0}\right)\right)\right\}$ and

$$
\min \{f(x), f(z), g(f(x), f(z))\} \leq f\left(x_{0}\right) \leq \max \{f(x), f(z), g(f(x), f(z))\} .
$$

Since $x, y, z, x_{0} \in K\left(x_{0}, r\right)$ we obtain the following inequalities of the adequate form as:

$f(x)-f\left(x_{0}\right) \leq \max \left\{f(y), f\left(x_{0}\right), g\left(f(y), f\left(x_{0}\right)\right)\right\}-\min \{f(x), f(z), g(f(x), f(z))\}$. 
and

$f(x)-f\left(x_{0}\right) \geq \min \left\{f(y), f\left(x_{0}\right), g\left(f(y), f\left(x_{0}\right)\right)\right\}-\max \{f(x), f(z), g(f(x), f(z))\}$.

Hence by the preceding inequality of the form (Os), for all points $x, y, z, x_{0} \in$ $K\left(x_{0}, r\right)$, we obtain the following inequalities in the form as

$$
-C\left\|x-x_{0}\right\|<f(x)-f\left(x_{0}\right)<C\left\|x-x_{0}\right\| .
$$

The preceding inequalities (4) proves the continuity of $f$ at a point $x_{0} \in D$. Whence, since $x_{0} \in D$ has been arbitrary, the continuity of $f$ in $D$ follows. The proof is complete.

In connection with the preceding facts, we have the following locally form of (Os). A general J-inner function $f: D \rightarrow \mathbb{R}$ for some function $g: f(D)^{2} \rightarrow$ $\mathbb{R}$ is called round locally oscillation at a point $x_{0} \in D$ iff there exists a neighbourhood $U \subset D$ of $x_{0}$ such that

$$
\begin{array}{r}
A:=\max \left\{f(y), f\left(x_{0}\right), g\left(f(y), f\left(x_{0}\right)\right)\right\}- \\
-\min \{f(x), f(z), g(f(x), f(z))\}<C\left\|x-x_{0}\right\|
\end{array}
$$

and

$$
\begin{array}{r}
B:=\min \left\{f(y), f\left(x_{0}\right), g\left(f(y), f\left(x_{0}\right)\right)\right\}- \\
-\max \{f(x), f(z), g(f(x), f(z))\}>-C\left\|x-x_{0}\right\|
\end{array}
$$

for some real constant $C>0$, and for all $x, y, z \in U$. A mapping $f: D \rightarrow \mathbb{R}$ is with the property of round local oscillation in $D$ iff it have the round locally oscillation at every point of $D$.

An immediate consequence of the preceding proof of Theorem 4 is the following statement.

Theorem 5a. Let $D \subset \mathbb{R}^{n}$ be a convex and open set, and let $f: D \rightarrow \mathbb{R}$ be a general J-inner function. If $f$ with the property of round local oscillation in $D$, then it is continuous in $D$.

This proof is totally analogous to the proof of the preceding statement.

From the preceding statement, we are now in a position to formulate the following consequence.

Corollary 2. (Bernstein-Doetsch, [1915]). Let $D \subset \mathbb{R}^{n}$ be a convex and open set, and let $f: D \rightarrow \mathbb{R}$ be a J-convex function. If $f$ is locally bounded above at a point of $D$, then it is continuous in $D$.

Proof. We notice that J-convex functions are general J-inner functions. Also, let $f$ be a locally bounded at an arbitrary point $x_{0} \in D$. Thus there exist positive constants $M$ and $r$ such that $K\left(x_{0}, r\right) \subset D$ and $|f(t)| \leq M$ for $t \in K\left(x_{0}, r\right)$. Then, the analogous to the preceding proof, for an arbitrary 
$x \in K\left(x_{0}, r\right)$ we have $\left\|x-x_{0}\right\|<r$, and we can find a $\lambda \in \mathbb{Q} \cap(0,1)$ such that

$$
\left\|x-x_{0}\right\| r^{-1}<\lambda<2\left\|x-x_{0}\right\| r^{-1},
$$

and that (1). Since $f$ is a J-convex function we obtain from Theorem 2 a the following two inequalities in the forms as

$$
f(x) \leq \lambda f(y)+(1-\lambda) f\left(x_{0}\right), \quad f\left(x_{0}\right) \leq(1+\lambda)^{-1} f(x)+\lambda(1+\lambda)^{-1} f(z)
$$

for all points $x, y, z \in K\left(x_{0}, r\right)$. Thus $f$ satisfies the conditions (Oa) and $(\mathrm{Ob})$, because

$$
A \leq \lambda f(y)+(1-\lambda) f\left(x_{0}\right)-f\left(x_{0}\right)=\lambda\left[f(y)-f\left(x_{0}\right)\right]<4 M r^{-1}\left\|x-x_{0}\right\|
$$

and

$$
\begin{aligned}
& \quad B \geq f(x)-(1+\lambda)^{-1} f(x)-\lambda(1+\lambda)^{-1} f(z)= \\
& =\lambda(1+\lambda)^{-1}[f(x)-f(z)]>-4 M r^{-1}\left\|x-x_{0}\right\| .
\end{aligned}
$$

This means that $f$ satisfy all the required hypotheses in Theorem 5a. Applying Theorem 5a to the mapping $f$ we obtain that it is continuous in $D$. The proof is complete.

Further, we shall introduce the concept of round locally boundness in $D \subset \mathbb{R}^{n}$. A general J-inner function $f: D \rightarrow \mathbb{R}$ for some bounded above function $g: f(D)^{2} \rightarrow \mathbb{R}$ with $M>0$ is called round locally bounded above at a point $x_{0} \in D$ iff there exists a neighbourhood $U \subset D$ of $x_{0}$ such that

$$
f(x) \leq M<\min \{f(y), f(z), g(f(y), f(z))\}+C\left\|x-x_{0}\right\|
$$

for some real constant $C>0$, and for all $x, y, z \in U$. A mapping $f: D \rightarrow \mathbb{R}$ is with the property of round local bounded above in $D$ iff it have the round locally bounded above at every point of $D$.

We are now in a position to formulate the following statement with who we precision, correction and expand an our former result.

Theorem 6. Let $D \subset \mathbb{R}^{n}$ be a convex and open set, and let $f: D \rightarrow \mathbb{R}$ be a general J-inner function for some bounded above function $g: f(D)^{2} \rightarrow \mathbb{R}$. If $f$ with the property of round local bounded above in $D$, then it is continuous in $D$.

Proof. Let $f$ be a round locally bounded above at a point of $D$. Take an arbitrary $x_{0} \in D$. Thus, there exists positive constants $M, C$ and $r$ such that (Bo) for all $x, y, z \in K\left(x_{0}, r\right) \subset D$, For an arbitrary $x \in K\left(x_{0}, r\right)$ we have $\left\|x-x_{0}\right\|<r$, and consequently we can find a $\lambda \in \mathbb{Q} \cap(0,1)$ such that $\left\|x-x_{0}\right\| r^{-1}<\lambda<2\left\|x-x_{0}\right\| r^{-1}$. From (1) and by Theorem 2a, since $f$ 
is general J-inner we obtain (2) and (3) for all $x, y, z \in K\left(x_{0}, r\right)$. Hence by (Bo) we obtain

$$
f(x)-f\left(x_{0}\right) \leq M-\min \{f(x), f(z), g(f(x), f(z))\}<C\left\|x-x_{0}\right\|
$$

and

$$
f(x)-f\left(x_{0}\right) \geq-\left(M-\min \left\{f(y), f\left(x_{0}\right), g\left(f(y), f\left(x_{0}\right)\right)\right\}\right)>-C\left\|x-x_{0}\right\|
$$

for all $x, y, z \in K\left(x_{0}, r\right)$. The preceding inequalities (5) and (6) proves the continu-ity of $f$ at a point $x_{0} \in D$. Whence, since $x_{0} \in D$ has been arbitrary, the continuity of $f$ in $D$ follows. The proof is complete.

Open problem 1. Let $\subset \mathbb{R}^{n}$ be a convex and open set, and let $f: D \rightarrow \mathbb{R}$ be a general J-inner function. If $f$ is locally oscillation at a point of $D$, does $f$ have the property of local oscillation in $D$ ?

\section{General convex functions}

In this section we continue the study of the general convex functions, which are introduced in the former paper by Tasković: Mat. Japonica, 37 (1992), 367-372. This section presents a new characterization of general convex functions in term of general level sets. Applications in convex analysis are considered.

Introduction and some results. In our former paper, have introduced the notion of general convex functions. A function $f: D \rightarrow \mathbb{R}$, where $\mathbb{R}$ denotes the real line and $D$ is a convex subset of $\mathbb{R}^{n}$, is said to be general convex if there is a function $g: f(D)^{2} \rightarrow \mathbb{R}$ such that

(Max)

$$
f(\lambda x+(1-\lambda) y) \leq \max \{f(x), f(y), g(f(x), f(y))\}
$$

for all $x, y \in D$ and for all $\lambda \in[0,1]$. We notice that the set of all convex and quasiconvex function can be a proper subset of the set all general convex functions.

In order, the function $g: \mathbb{R}^{2} \rightarrow \mathbb{R}$ is increasing if $x_{i}, y_{i} \in \mathbb{R}$ and $x_{i} \leq y_{i}$ $(i=1,2)$ implies $g\left(x_{1}, x_{2}\right) \leq g\left(y_{1}, y_{2}\right)$. On the other hand, the function $g: \mathbb{R}^{2} \rightarrow \mathbb{R}$ is level increasing if it is increasing and with the property

$$
g(\max \{x, g(x, x)\}, \max \{x, g(x, x)\}) \leq \max \{x, g(x, x)\}
$$

for every $x \in \mathbb{R}$. Geometrically see Figures 1, 2 and 3. Further see: ReddyMukherjee [2001].

It is well-known that a convex function can be characterized by convexity of its epigraph. Also, we know that a quasiconvex function can be characterized by convexity of its level sets.

In this paper we present a new characterization of general convex functions as convexity of their general level sets. In this sense, we are now in a position to formulate main general statement. 


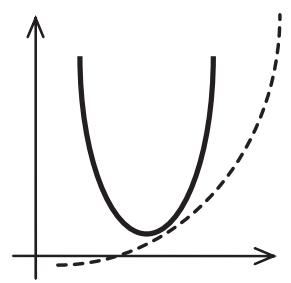

FiguRE 1

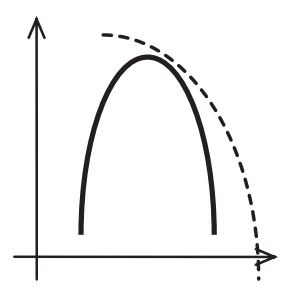

FiguRE 2

Theorem 7. Let $D \subset \mathbb{R}^{n}$ be a convex and open set. The function $f: D \rightarrow \mathbb{R}$ is general convex for some level increasing function $g: \mathbb{R}^{2} \rightarrow \mathbb{R}$ if and only if

(GI) $\quad g\left(D_{a}\right):=\{x \in D \mid \max \{f(x), g(f(x), f(x))\} \leq \max \{a, g(a, a)\}\}$

is a convex set for each number $a \in \mathbb{R}$.
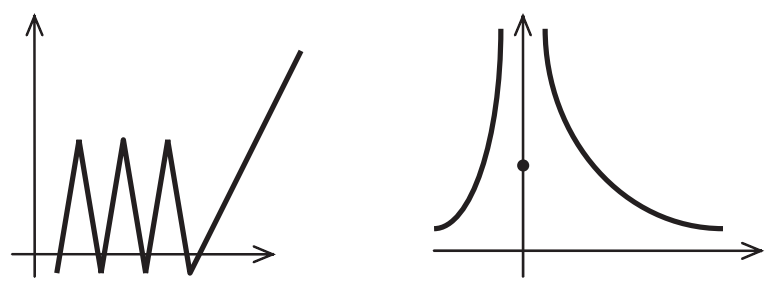

Figure 3

Proof. Suppose that $f$ is a general convex function, and let $x, y \in g\left(\left\{D_{a}\right\}\right)$. Therefore $x, y \in D$ and

(7)

$\max \{f(x), g(f(x), f(x))\}, \max \{f(y), g(f(y), f(y))\} \leq \max \{a, g(a, a)\}$.

Let $z=\lambda x+(1-\lambda) y$ for $\lambda \in[0,1]$. By convexity of $D$ we obtain $z \in D$. Furthemore, by general convexity of $f$, i.e., from (Max) and (7) we have

$$
\begin{gathered}
f(z) \leq \max \{f(x), f(y), g(f(x), f(y))\} \leq \\
\leq \max \{f(x), f(y), \max (g(f(x), f(x)), g(f(y), f(y)))\} \leq \max \{a, g(a, a)\} .
\end{gathered}
$$

Thus $f(z) \leq \max \{a, g(a, a)\}$ and from level increasing of $g: \mathbb{R}^{2} \rightarrow \mathbb{R}$ we obtain $g(f(z), f(z)) \leq g(\max \{a, g(a, a)\}, \max \{a, g(a, a)\} \leq \max \{a, g(a, a)\}$. This mean that is $\max \{f(z), g(f(z), f(z))\} \leq \max \{a, g(a, a)\}$, i.e., $z \in$ $g\left(D_{a}\right)$. Thus $g\left(D_{a}\right)$ is a convex set.

Conversely, suppose that $g\left(D_{a}\right)$ is a convex set for each number $a \in \mathbb{R}$. Let $z=\lambda x+(1-\lambda) y$ for all $\lambda \in[0,1]$. Notice that $x, y \in g\left(\left\{D_{a}\right\}\right)$ for 
$\max \{a, g(a, a)\}=\max \{f(x), f(y), g(f(x), f(y))\}$. By assumption, $g\left(\left\{D_{a}\right\}\right)$ is convex, so that $z \in g\left(\left\{D_{a}\right\}\right)$. Therefore,

$$
\begin{gathered}
f(z) \leq \max \{f(z), g(f(z), f(z))\} \leq \\
\leq \max \{a, g(a, a)\}=\max \{f(x), f(y), g(f(x), f(y))\} .
\end{gathered}
$$

Hence, $f$ is a general convex function. The proof is complete. We notice, from the preceding proof of Theorem 7 as an immediate fact we obtain the following statement.

Corollary 3. Let $D \subset \mathbb{R}^{n}$ be a convex and open set, and let $f: D \rightarrow \mathbb{R}$. If there is a function $g: \mathbb{R}^{2} \rightarrow \mathbb{R}$ such that the sets $g\left(D_{a}\right)$ are convex, then $f$ is a general convex function.

On the other hand, from the preceding statement, we are now in a position to formulate the following consequence for quasiconvex functions.

In this sense, a function $f: D \rightarrow \mathbb{R}$, where $D$ is a convex subset of $\mathbb{R}^{n}$, is said to be quasiconvex if

$$
f(\lambda x+(1-\lambda) y) \leq \max \{f(x), f(y)\}
$$

for all $x, y \in D$ and for arbitrary $\lambda \in[0,1]$. We notice that the set of all quasiconvex function can be a proper subset of the set all general convex functions.

Corollary 4. (De Finetti [1949], Fenchel [1949]). Let $D \subset \mathbb{R}^{n}$ be a convex and open set. The function $f: D \rightarrow \mathbb{R}$ is quasiconvex if and only if

$$
L_{a}:=\{x \in D \mid f(x) \leq a\}
$$

is a convex set for each number $a \in \mathbb{R}$. (The set $L_{a}$ is called level set).

Proof. If to teasing on the quasiconvex class functions taking $g(f(x), f(y))=$ $\max \{f(x), f(y)$ from Theorem 7 we obtain directly this statement for quasiconvex functions and level sets. The proof is complete.

Further, as an immediate consequence of Theorem 7 we obtain directly the following statement with which we precision an our former result.

Corollary 5. (Extremal principle, Tasković [1992b]). Let $X$ be a reflexive Banach space and let $M$ be a nonempty, closed, bounded and convex set in $X$. If $f: M \rightarrow \mathbb{R} \cup\{+\infty\}$ is a general convex function for some continuous level increasing function $g: \mathbb{R}^{2} \rightarrow \mathbb{R}$ and if the set $g\left(D_{a}\right)$ is closed for all $a \in \mathbb{R}$, then $f$ has a minimum on $M$.

Proof. The set $M$ is weakly compact, because $M$ is bounded, closed and convex set in reflexive Banach space $X$. Further, $g\left(D_{a}\right)$ is closed and convex (from Theorem 7), and hence weakly closed. Therefore $f$ is lower semicontinuous in the weak topology on $M$. The conclusion now follows from Weierstrass theorem. The proof is complete. 
Further applications. We now give a result which shows that the maximum of a general convex function over a compact polyhedral set occurs at an extreme point.

A nonempty set $D \subset \mathbb{R}^{n}$ is called a polyhedral set if it is the intersection of a finite number of closed half spaces. Note that a polyhedral set is a closed convex set. A vector $z \in D$ is called an extreme point of $D$ if $z=\lambda x+(1-\lambda) y$ with $\lambda \in(0,1)$ and $x, y \in D$ implies that $z=x=y$.

Theorem 8. Let $D \subset \mathbb{R}^{n}$ be a nonempty compact polyhedral set, and let $f: D \rightarrow \mathbb{R}$ be a continuous and general convex function for some level increasing function $g: \mathbb{R}^{2} \rightarrow \mathbb{R}$. Consider the problem to maximize $f(x)$ subject to $x \in D$. Then there exists an optimal solution $\xi \in D$ to the problem which is an extreme point of $D$.

Proof. Note that $f$ is continuous on $D$ and hence attains a maximum, say, at $\xi \in D$. If there is an extreme point whose objective is equal to $f(\xi)$, then the result is at hand. Otherwise, let $x_{1}, \ldots, x_{k}$ be the extreme points of $D$, and assume that $f(\xi)>f\left(x_{j}\right)$ for $j=1, \ldots, k$. By representation of points in $D, \xi \in D$ can be represented as $\xi=\lambda_{1} x_{1}+\cdots+\lambda_{k} x_{k}$, where $\lambda_{1}+\cdots+\lambda_{k}=1$ for $\lambda_{j} \geq 0(j=1, \ldots, k)$. Since $f(\xi)>f\left(x_{j}\right)$ for each $j=1, \ldots, k$ we obtain

$$
f(\xi)>\max _{j=1, \ldots, k} f\left(x_{j}\right):=\max \{a, g(a, a)\} .
$$

Now consider the sets $g\left(D_{a}\right)$ with (G1) for some level increasing function $g: \mathbb{R}^{2} \rightarrow \mathbb{R}$. Note that $x_{j} \in g\left(D_{a}\right)$ for $j=1, \ldots, k$, and by general convexity of $f$ (Theorem 1) the set $g\left(D_{a}\right)$ is convex. Hence, $\xi=\lambda_{1} x_{1}+\cdots+\lambda_{k} x_{k}$ belongs to $g\left(D_{a}\right)$, i.e.,

$$
\max \{f(\xi), g(f(\xi), f(\xi))\} \leq \max \{a, g(a, a)\} .
$$

This implies that $f(\xi) \leq \max \{a, g(a, a)\}$, which contradicts (8). This contra-diction shows that $f(\xi)=f\left(x_{j}\right)$ for some extreme point $x_{j}$. The proof is complete.

We notice that quasiconvex functions are, de facto, general convex functions. Thus we obtain directly as an immediate consequence of Theorem 8 and corresponding result for quasiconvex functions. This mean that the maximum of a quasiconvex function over a compact polyhedral set occurs at an extreme point.

General level sets. In what follows we assume that $D$ is nonempty convex subset of $\mathbb{R}^{n}$ and $\varepsilon$ is a positive constant. Recall that a function $f: D \rightarrow \mathbb{R}$ is said to be $\varepsilon$-quasiconvex if

$$
f(\lambda x+(1-\lambda) y) \leq \max \{f(x), f(y)\}+\varepsilon
$$

for all $x, y \in D$, and all $\lambda \in[0,1]$. For $\varepsilon=0$ this definition reduces to that of quasiconvex function, cf. Roberts-Varberg [1973]. 
Recall that a function $f: D \rightarrow \mathbb{R}$ is said to be $\varepsilon$-general convex if for some $\varepsilon>0$ there is a function $g: f(D)^{2} \rightarrow \mathbb{R}$ such that

$$
f(\lambda x+(1-\lambda) y) \leq \max \{f(x), f(y), g(f(x), f(y))\}+\varepsilon
$$

for all $x, y \in D$, and all $\lambda \in[0,1]$. For $\varepsilon=0$ this definition reduces to that of general convex function.

On the other hand, the function $g: \mathbb{R}^{2} \rightarrow \mathbb{R}$ is $\varepsilon$-level increasing if it is increasing and with the property

$$
g(\max \{x, g(x, x)\}+\varepsilon, \max \{x, g(x, x)\}+\varepsilon) \leq \max \{x, g(x, x)\}+\varepsilon
$$

for every $x \in \mathbb{R}$ and $\varepsilon>0$.

Assumme that $f: D \rightarrow \mathbb{R}$ is a $\varepsilon$-general convex function for some $\varepsilon$-level increasing function $g: \mathbb{R}^{2} \rightarrow \mathbb{R}$ and consider the general level sets

$$
g\left(L_{a}\right):=\{x \in D \mid \max \{f(x), g(f(x), f(x)) \leq a\}
$$

for $a \in \mathbb{R}$. It is clear that $\bigcup_{a \in \mathbb{R}} g\left(L_{a}\right)=D$ and $g\left(L_{a}\right) \subset g\left(L_{b}\right)$ whenever $a<b$. We notice, the set $g\left(L_{a}\right)$ is called general level set.

We are now in a position to formulate the following statement with which we precision and expand a fact (a comment) in 1992.

Theorem 9. Let $D \subset \mathbb{R}^{n}$ be a nonempty convex set, and let $f: D \rightarrow \mathbb{R}$ be a $\varepsilon$-general convex function for some $\varepsilon$-level increasing function $g: \mathbb{R}^{2} \rightarrow \mathbb{R}$. If $x_{1}, \ldots, x_{m+1} \in g\left(L_{a}\right)$ for $m \in \mathbb{N}, a \in \mathbb{R}$, and $\lambda_{1}+\cdots+\lambda_{m+1}=1$ $\left(\lambda_{1}, \ldots, \lambda_{m+1} \in[0,1]\right)$, then

$$
\lambda_{1} x_{1}+\cdots+\lambda_{m+1} x_{m+1} \in g\left(L_{\max \{a, g(a, a)\}+\varepsilon k(m)}\right),
$$

where $k(m)=1+\left[\log _{2} m\right]$. (For this result and further facts see: Tasković [2005].)

Proof. If $x, y \in g\left(L_{a}\right)$ and $\lambda_{1}+\lambda_{2}=1\left(\lambda_{1}, \lambda_{2} \in[0,1]\right)$ we have $\max \{f(x)$, $g(f(x), f(x))\} \leq a$, and $\max \{f(y), g(f(y), f(y))\} \leq a$. From inequality (M) for $z=\lambda_{1} x+\lambda_{2} y$ we obtain $f(z) \leq \max \{f(x), f(y), g(f(x), f(y))\}+\varepsilon \leq$ $\max \{a, g(a, a)\}+\varepsilon$. By $\varepsilon$-level increasing of $g: \mathbb{R}^{2} \rightarrow \mathbb{R}$ we obtain $g(f(z), f(z)) \leq g(\max \{a, g(a, a)\}+\varepsilon, \max \{a, g(a, a)\}+\varepsilon) \leq \max \{a, g(a, a)\}+\varepsilon$.

This means that $\max \{f(z), g(f(z), f(z))\} \leq \max \{a, g(a, a)\}+\varepsilon$, i.e., $z=$

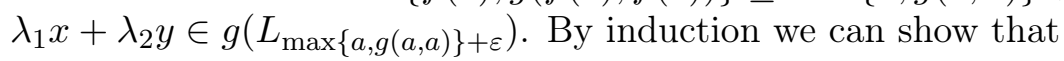

$$
\lambda_{1} x_{1}+\cdots+\lambda_{2^{r}} x_{2^{r}} \in g\left(L_{\max \{a, g(a, a)\}+r \varepsilon}\right),
$$

for all $r \in \mathbb{N}$, for $x_{1}, \ldots, x_{2^{r}} \in D$ and $\lambda_{1}, \ldots, \lambda_{2^{r}} \in[0,1]$ with $\lambda_{1}+\cdots+\lambda_{2^{r}}=$ 1. Fix an $m \in \mathbb{N}$ and assume that $x_{1}, \ldots, x_{m} \in D$ with $\lambda_{1}, \ldots, \lambda_{m} \in[0,1]$ and $\lambda_{1}+\cdots+\lambda_{m}=1$. Take the minimal $r \in \mathbb{N}$ such that $m+1 \leq 2^{r}$. One can easily check that $r=\left[\log _{2} m\right]+1:=k(m)$. In the case $m+1<2^{r}$, let us 
put $\lambda_{m+2}=\cdots=\lambda_{2^{r}}=0$ and $x_{m+2}=\cdots=x_{2^{r}}:=x_{1}$. Then by preceding facts and (3), we obtain

$$
\lambda_{1} x_{1}+\cdots+\lambda_{m+1} x_{m+1}=\lambda_{1} x_{1}+\cdots+\lambda_{2^{r}} x_{2^{r}} \in g\left(L_{\max \{a, g(a, a)\}+\varepsilon k(m)}\right) .
$$

From Theorem 9 we are now in a position to formulate the following directly consequence for quasiconvex functions.

Corollary 6. (Nikodem, [1988]). Let $D \subset \mathbb{R}^{n}$ be a nonempty convex set, and let $f: D \rightarrow \mathbb{R}$ be a $\varepsilon$-quasiconvex function. If $x_{1}, \ldots, x_{m+1} \in L_{a}$ for $m \in \mathbb{N}, a \in \mathbb{R}$, and $\lambda_{1}+\cdots+\lambda_{m+1}=1\left(\lambda_{1}, \ldots, \lambda_{m+1} \in[0,1]\right)$, then

$$
\lambda_{1} x_{1}+\cdots+\lambda_{m+1} x_{m+1} \in L_{a+\varepsilon k(m)},
$$

for $k(m):=1+\left[\log _{2} m\right]$.

Proof. If to teasing on the $\varepsilon$-quasiconvex class functions taking $g(f(x), f(y))=$ $\max \{f(x), f(y)\}$ from Theorem 9 we obtain directly this statement, because in this case $g\left(L_{a}\right)=L_{a}$. The proof is complete.

Annotations. A function $f:[a, b] \rightarrow \mathbb{R}$ is said to be convex if and only if each shord lies above the corresponding arc of the curve. In contrast to arbitrary real functions, convex functions possess a number of remarkable properties of which we list two here: 1) if $f$ has a local minimum at $x_{0}$, then $f$ also has a global minimum at $x_{0}$; and 2) If $f^{\prime}$ exists on $[a, b]$, then on $[a, b]: f$ is convex if and only if $f^{\prime}$ is monotonely increasing.

The property 2) yields the connection between convex functionals and monotone operators. A convex function possesses only minima as critical points. If $f:[a, b] \rightarrow \mathbb{R}$ is a convex but not necessarily differentiable function, then a global minimum at $x_{0}$ can be characterized by $0 \in \partial f\left(x_{0}\right)$ instead of by $f^{\prime}\left(x_{0}\right)=0$ or $f^{\prime}\left(x_{0}\right)\left(x-x_{0}\right) \geq 0$ for all $x \in[a, b]$.
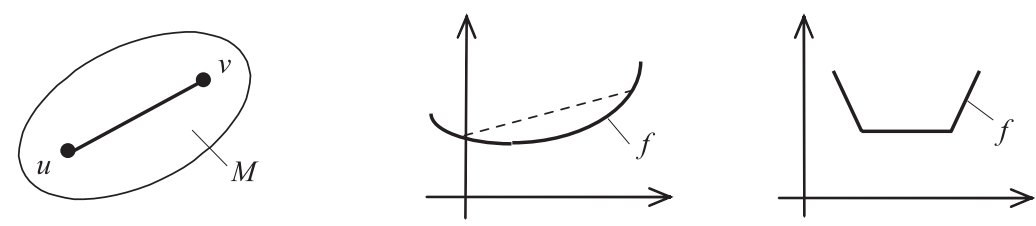

FIGURE 4

Here, the so-called subdifferential $\partial f\left(x_{0}\right)$ equals the set of all slopes $m$ of the straight lines through $\left(x_{0}, f\left(x_{0}\right)\right)$ which lie beneath the curve determined by $f$ is the starting point for the convex analysis that we develop in the chapter 5. For convex sets and convex functions see Figs. 4 and 5. 

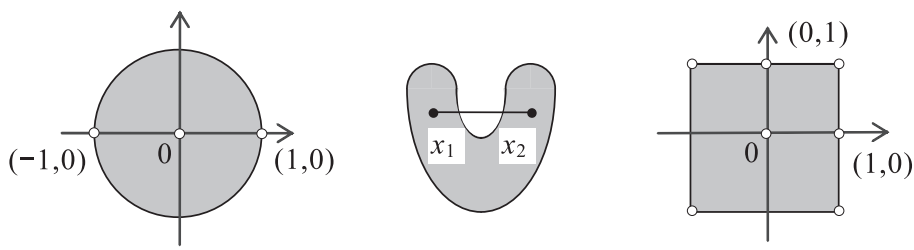

\section{FIGURE 5}

\section{General Hahn-Banach theorem}

In this section we give some generalizations of the well known HahnBanach theorem in terms of general convex functions.

Let $A \subset X$ be a subset of linear space $X$. We say that $A$ is $Q$-radial at a point $a \in A$ if for every $y \in X(y \neq 0)$, there exists an $\varepsilon_{y}>0$ such that $a+\lambda y \in A$ for every $\lambda \in \mathbb{Q} \cap\left(0, \varepsilon_{y}\right)$.

Lemma 3. Let $X$ be a linear space, let $D \subset X$ be a set $Q$-convex and $Q$ radial at a point $x_{0} \in D$, and let $L \subset X$ be a linear space (over $\mathbb{Q}$ ) such that $x_{0} \in L$. Let $f: D \rightarrow \mathbb{R}$ be a function fulfilling the inequality

$$
f(\lambda x+(1-\lambda) y) \leq \max \{f(x), f(y), g(f(x), f(y))\}
$$

for a function $g: f(D)^{2} \rightarrow \mathbb{R}$, for all $x, y \in D$ and for every $\lambda \in \mathbb{Q} \cap[0,1]$. If $z \notin L$, if $Z=\operatorname{Lin}(L \cup\{z\})$ and if $h: L \rightarrow \mathbb{R}$ is a linear functional such that

$$
h(x) \leq f(x) \quad \text { for every } \quad x \in D \cap L,
$$

then there exists a linear functional $H: Z \rightarrow \mathbb{R}$ such that the following inequality holds in the form as

$$
H(x) \leq f(x) \quad \text { for every } \quad x \in D \cap Z
$$

and $H \mid L=h$, i.e., there is an extension of the linear functional $h$ on $Z$.

Hence we derive the rational version of the Hahn-Banach theorem for the general convex functions in the form as.

Theorem 10. Let $X$ be a linear space, let $D \subset X$ be a set $Q$-convex and $Q$-radial at a point $x_{0} \in D$, and let $L \subset X$ be a linear space (over $\mathbb{Q}$ ) such that $x_{0} \in L$. Let $f: D \rightarrow \mathbb{R}$ be a function fulfilling $(Q)$ for all $x, y \in D$ and for every $\lambda \in \mathbb{Q} \cap[0,1]$. If $h: L \rightarrow \mathbb{R}$ is a linear functional with property (10), then there exists a linear functional $H: X \rightarrow \mathbb{R}$ such that

$$
H(x) \leq f(x) \quad \text { for every } \quad x \in D
$$

and $H \mid L=h$, i.e., there is an extension of the linear functional $h$ on the space $X$.

In this section arguing as in the proof of Theorem 10 we can get however the following result. 
Theorem 11. (General Hahn-Banach theorem). Let $X$ be a real linear space, let $D \subset X$ be a subspace and let $f: X \rightarrow \mathbb{R}$ be a general convex function such that

$$
h(x) \leq f(x) \quad \text { for every } \quad x \in D,
$$

where $h: D \rightarrow \mathbb{R}$ is a linear functional. Then there is a linear functional $H: X \rightarrow \mathbb{R}$ such that

$$
H(x) \leq f(x) \quad \text { for every } \quad x \in X
$$

and $H \mid D=h$, i.e., there is a linear extension of the linear functional $h$ on $X$.

By the preceding method, we also prove the following result, which is a separation theorem on a linear space.

Theorem 12. (Separation of concavity and general convexity). Let $E$ be a real linear space, let $D \subset E$ be a nonempty convex subset, and let $f: E \rightarrow \mathbb{R}$ be a general convex with tent function such that

$$
k(x) \leq f(x) \quad \text { for every } \quad x \in D,
$$

where $k: D \rightarrow \mathbb{R}$ is a concave functional. Then there is a linear functional $H: E \rightarrow \mathbb{R}$ such that

$$
k(x) \leq H(x) \quad \text { for every } \quad x \in D,
$$

and

$$
H(y) \leq f(y) \quad \text { for every } y \in E .
$$

For the proof of this statement the following result is essential.

Lemma 4. Let $X$ be a nonempty compact convex subset of a separated linear topological space and $f_{v}: X \rightarrow \mathbb{R} \cup\{+\infty\}$ for $v \in I$ a family of lower semicontinuous general convex with tent functionals. If for any finite indices $v_{1}, \ldots, v_{n}$ the following inequality holds

$$
\lambda_{1} f_{v_{1}}(y)+\cdots+\lambda_{n} f_{v_{n}}(y) \leq 0
$$

for every $y \in X$ and for any nonnegative numbers $\lambda_{1}, \ldots, \lambda_{n}$ with property $\lambda_{1}+\cdots+\lambda_{n}=1$, then there is an $x \in X$ such that

$$
f_{v}(x) \leq 0 \quad \text { for every } \quad v \in I .
$$

Let $E$ be a linear functional and let $f: E \rightarrow \mathbb{R}$ be a general convex with tent function. For two nonempty subsets $A$ and $B$ of $E$, we consider a number

$$
f(A, B):=\inf \{f(x-y): x \in A, y \in B\} .
$$

As a directly consequence of the preceding results we obtain the following statement. 
Proposition 7. Let $E$ be a real linear space and let $f: E \rightarrow \mathbb{R}$ be a general convex with tent function. If $C$ and $D$ are nonempty convex subsets of $E$ such that $f(C, D)>-\infty$, then there is a linear functional $H: E \rightarrow \mathbb{R}$ such that

$$
\inf \{H(x): x \in C\}=f(C, D)+\sup \{H(y): y \in D\}
$$

and

$$
H(x) \leq f(x) \quad \text { for every } \quad x \in E .
$$

Let $X$ be a real linear space, $D$ a convex set in the space $X, k: D \rightarrow \mathbb{R}$ and $f: D \rightarrow \mathbb{R}$ are given functionals, $k_{\beta}: D \rightarrow \mathbb{R}(\beta \in J)$ and $f_{\alpha}: D \rightarrow \mathbb{R}$ $(\alpha \in I)$ denote the sequences of concave and general convex functionals such that $k(x) \leq\left\{k_{\beta}\right\}(x), f_{\alpha}(x) \leq f(x)$ for all $x \in D$, respectively. Also, let the envelope (of functional $f$ ) $\bar{f}(x)=\sup _{\alpha \in I} f_{\alpha}(x)$ be a general convex with tent function. If the functionals $k: D \rightarrow \mathbb{R}$ and $f: X \rightarrow \mathbb{R}$ have the preceding properties we call that $k$ and $f$ have the envelope majorantes property.

Theorem 12a. Let $X$ be a real linear space, let $D \subset X$ be a nonempty convex set, and let $k: D \rightarrow \mathbb{R}$ and $f: X \rightarrow \mathbb{R}$ have the envelope majorantes property. Then there is a linear functional $H: X \rightarrow \mathbb{R}$ such that

$$
\begin{aligned}
& k(x) \leq H(x) \quad \text { for every } \quad x \in D, \\
& H(x) \leq f(x) \quad \text { for every } \quad x \in X
\end{aligned}
$$

if and only if the following inequality holds

$$
\inf _{\beta \in J} K_{\beta}(x) \leq \inf _{\lambda>0} \frac{1}{\lambda} \sup _{\alpha \in I} f_{\alpha}(\lambda x)
$$

for every $x \in \operatorname{Conv} D$, where the functions $K_{\beta}(x)$ are defined by

$$
K_{\beta}(x)=\left\{\begin{array}{lll}
k_{\beta}(x) & \text { for every } & x \in D, \\
-\infty & \text { for every } & x \in \operatorname{Conv} D) \backslash D .
\end{array}\right.
$$

Theorem 13. (Extension of Mazur-Orlicz's theorem). Let $X$ be a real linear space, and let $g: X \rightarrow \mathbb{R}$ be a given functional. If $J$ is an arbitrary index set, $\left\{x_{j}: j \in J\right\} \subset X$, and if $\left\{c_{j}: j\right\} \in J \subset \mathbb{R}$, then the system of form

$$
\phi \leq g, \quad c_{j} \leq \phi\left(x_{j}\right) \quad \text { for } \quad j \in J
$$

has a solution $\phi \in X^{*}$ if and only if for every finite set $\{j(1), \ldots, j(n)\} \subset J$ and for every set $\left\{\alpha_{1}, \ldots, \alpha_{n}\right\}$ of nonnegative numbers we have

$$
\sum_{k=1}^{n} \alpha_{k} c_{j(k)} \leq g\left(\sum_{k=1}^{n} \alpha_{k} x_{j(k)}\right) .
$$


If $g: X \rightarrow \mathbb{R}$ is a sublinear functional, then from Theorem 13 we obtain well known result in 1934 of S.Mazur and W. Orlicz.

In this section we will consider a stronger version of the general HahnBanach theorem which will also turn out to be equivalent to the Axiome of Choice.

Theorem 14. (An equivalent of Axiom of Choice). Let $M$ be a subspace of real linear space $E$ and $S$ a subset of $E$. Suppose $f: E \rightarrow \mathbb{R}$ is a general convex functional and $h: M \rightarrow \mathbb{R}$ a linear functional such that

$$
h(x) \leq f(x) \quad \text { for every } \quad x \in M .
$$

Then the set $G$ of all $f$-dominated linear extensions of $h$ to $E$ has an element $g: E \rightarrow \mathbb{R}$ such that for all $H \in G$ with $g(s) \leq H(s)$ for all $s \in S$, we have $g(s)=H(s)$ for all $s \in S$. That is, $g$ is $S$-maximal in $G$.

\section{A SANDWich With General CONVEXity}

In is the aim of this section to characterize which can be separated by a general convex function. This leads us to functional inequality for real functions $f, g: I \rightarrow \mathbb{R}(I \subset \mathbb{R}$ is an interval $)$ such that

$$
f(\lambda x+(1-\lambda) y) \leq \max \{g(x), g(y), G(g(x), g(y))\}
$$

for a continuous increasing function $G: g(I)^{2} \rightarrow \mathbb{R}$, for all $x, y \in I$ and for arbitrary $\lambda \in[0,1]$.

Theorem 15. (Sandwich Theorem). Real functions $f, g: I \rightarrow \mathbb{R}(I \subset \mathbb{R}$ is an interval) satisfy $(R)$ for all $x, y \in I$ and arbitrary $\lambda \in[0,1]$ if and only if there exists a general convex function $h: I \rightarrow \mathbb{R}$ for a continuous increasing function $S: h(I)^{2} \rightarrow \mathbb{R}$ such that

$$
f(x) \leq h(x) \leq g(x) \quad \text { for every } \quad x \in I .
$$

Arguing as in the proof of Theorem 15, by Tasković [2001], we can get however the following result.

Theorem 16. (Tasković, [2001]). Real functions $f, g: D \rightarrow \mathbb{R}$ ( $D$ is a convex subset of a vector space) satisfy for a continuous increasing function $G: g(D)^{n} \rightarrow \mathbb{R}$ the following inequality

$$
f\left(\sum_{j=1}^{n} \lambda_{j} x_{j}\right) \leq \max \left\{g\left(x_{1}\right), \ldots, g\left(x_{n}\right), G\left(g\left(x_{1}\right), \ldots, g\left(x_{n}\right)\right)\right\}
$$

for all $n \in \mathbb{N}$, for all $x_{1}, \ldots, x_{n} \in D$ and for reals $\lambda_{1}, \ldots, \lambda_{n} \in[0,1]$ with property $\lambda_{1}+\cdots+\lambda_{n}=1$ if and only if there exists a general convex function $h: D \rightarrow \mathbb{R}$ for a continuous increasing function $S: h(D)^{n} \rightarrow \mathbb{R}$ such that

$$
f(x) \leq h(x) \leq g(x) \quad \text { for every } \quad x \in D .
$$


We notice, if real functions $f$ and $g$, defined on a convex subset $D$ of an $(n-1)$-dimensional real linear space, satisfy (11) if and only if there exists a general convex function $h: D \rightarrow \mathbb{R}$ satisfying (12).

Further applications of Theorem 15 concern solutions of the inequalities for the function $f: J \rightarrow \mathbb{R}\left(J\right.$ either $\mathbb{R}_{+}^{0}$ or $\left.\mathbb{R}\right)$ with

$$
f(\lambda x+(T-\lambda) y) \leq \max \{f(x), f(y), G(f(x), f(y))\}
$$

for a continuous increasing function $g: f(J)^{2} \rightarrow \mathbb{R}$, for all $x, y \in J$, for given $T>0$ and for arbitrary $\lambda \in[0, T]$.

For given $T>0$ and $f: J \rightarrow \mathbb{R}$ we define the function $\left\{f_{T}\right\}: J \rightarrow \mathbb{R}$ by the following formula in the form as

$$
f_{T}(x)=T^{-1} f(T x) .
$$

Theorem 17. (Taković, [2001]). Let T be a positive real number. A function $f: J \rightarrow \mathbb{R}$ satisfies (13) for all $x, y \in J$ and arbitrary $\lambda \in[0, T]$ if and only if there exists a general convex function $\psi: J \rightarrow \mathbb{R}$ for a continuous increasing function $S: \psi(J)^{2} \rightarrow \mathbb{R}$ such that

$$
\psi_{T}(x) \leq f(x) \leq \psi(x) \quad \text { for every } \quad x \in J .
$$

Let $f: I \rightarrow \mathbb{R}(I \subset \mathbb{R}$ is an interval $)$ for a continuous increasing function $G: f(I)^{2} \rightarrow \mathbb{R}$ satisfy the following inequality

$$
f(\lambda x+(T-\lambda) y+(1-T) \xi) \leq \max \{f(x), f(y), G(f(x), f(y))\}
$$

for all $x, y \in I$, for given $T \in(0,1)$, for arbitrary $\lambda \in[0, T]$ and for fixed $\xi \in I$. Fix a real interval $I$ and a point $\xi \in I$. For $T \in(0,1)$ put

$$
I_{T}^{*}=T I+(1-T) \xi
$$

Given a real function $\psi$ with the domain containing $I_{T}^{*}$, we define $\psi_{T}^{*}$ : $I \rightarrow \mathbb{R}$ by the following formula in the form as

$$
\psi_{T}^{*}(x)=T^{-1}(\psi(T x+(1-T) \xi)-(1-T) \psi(\xi)) .
$$

Theorem 18. Let $T \in(0,1)$. A function $f: I \rightarrow \mathbb{R}(I \subset \mathbb{R}$ is an interval $)$ satisfies (14) for all $x, y \in I$ and arbitrary $\lambda \in[0, T]$ if and only if there exists a general convex function $\psi: I_{T}^{*} \rightarrow \mathbb{R}$ for a continuous increasing function $S: \psi\left(I_{T}^{*}\right)^{2} \rightarrow \mathbb{R}$ such that

$$
\begin{cases}\psi_{T}^{*}(x) \leq f(x) \quad \text { for every } x \in I, \text { and } \\ f(x) \leq \psi(x) & \text { for every } x \in I_{T}^{*}\end{cases}
$$




\section{Characterizations of General Convexity}

A general convex function $f: D \rightarrow \mathbb{R}$ is said to be general convex with contact if (Max) holds, if there are $x_{0}, y_{0} \in D$ such that $f\left(x_{0}\right)=f\left(y_{0}\right)=$ $g\left(f\left(x_{0}\right), f\left(y_{0}\right)\right)$, and if for $x_{1}<y_{1}<z_{1}$

$(\mathrm{Mm})$

$\min \left\{f\left(x_{1}\right), f\left(z_{1}\right), g\left(f\left(x_{1}\right), f\left(z_{1}\right)\right)\right\}<\max \left\{f\left(x_{1}\right), f\left(z_{1}\right), g\left(f\left(x_{1}\right), f\left(z_{1}\right)\right)\right\}$

implies that there are $a_{1}, a_{2} \in D$ such that

$$
f\left(x_{0}\right)<\min \left\{f\left(a_{1}\right), f\left(a_{2}\right), g\left(f\left(a_{1}\right), f\left(a_{2}\right)\right)\right\}
$$

or there are $b_{1}, b_{2} \in D$ such that

$$
\max \left\{f\left(b_{1}\right), f\left(b_{2}\right), g\left(f\left(b_{1}\right), f\left(b_{2}\right)\right)\right\}<f\left(x_{0}\right) .
$$

Also, a function $f: D \rightarrow \mathbb{R}$ is said to be in contact with a function $g: f(D)^{2} \rightarrow \mathbb{R}$ if $f\left(x_{0}\right)=f\left(y_{0}\right)=g\left(f\left(x_{0}\right), f\left(y_{0}\right)\right)$ for some $x_{0}, y_{0} \in D$, and if $(\mathrm{Mm})$ implies $(\mathrm{U})$ or $(\mathrm{V})$; if $g$ is a continuous function, then $f$ is said to be in continuous contact.

We are now in a position to formulate our the following characterizations for general convex functions.

Theorem 19. (Monotonity of quotients). Let $J \subset \mathbb{R}$ be an open interval, and let $f: J \rightarrow \mathbb{R}$ be a function in contact with a function $g: f(J)^{2} \rightarrow \mathbb{R}$. Then each of the following conditions (postulated for every $x, y, z \in J$ with $x<y<z)$ is necessary and sufficient for the function $f$ to be general convex with contact $g$ :

$$
\begin{gathered}
f(y) \leq \max \{f(x), f(z), g(f(x), f(z))\}, \\
\frac{2 f(y)-f(x)-\max \{f(x), f(z), g(f(x), f(z))\}}{y-x} \leq \\
\quad \leq \frac{2 f(z)-f(x)-\max \{f(x), f(z), g(f(x), f(z))\}}{z-x}, \\
\frac{2 f(z)-f(x)-\max \{f(x), f(z), g(f(x), f(z))\}}{z-x} \leq \\
\quad \leq \frac{2 f(z)-f(y)-\max \{f(x), f(z), g(f(x), f(z))\}}{z-y} .
\end{gathered}
$$

Also, we are now in a position to formulate our the following characterizations for general convex functions.

Theorem 20. (Monotonity of quotients). Let $J \subset \mathbb{R}$ be an open interval, and let $f: J \rightarrow \mathbb{R}$ be a function in contact with a function $g: f(J)^{2} \rightarrow \mathbb{R}$. Then each of the following conditions (postulated for arbitrary $a, b \in J$ and for every $x, y, z \in J$ with $x<y<z)$ is necessary and sufficient for the 
function $f$ to be general convex with contact $g$ :

$$
\begin{gathered}
f(\xi) \leq \max \{f(a), f(b), g(f(a), f(b))\} \quad \text { for all } \xi \in[a, b], \\
\frac{2 f(y)-f(x)-\max \{f(a), f(b), g(f(a), f(b))\}}{y-x} \leq \\
\leq \frac{2 f(z)-f(x)-\max \{f(a), f(b), g(f(a), f(b))\}}{z-x}, \\
\frac{2 f(z)-f(x)-\max \{f(a), f(b), g(f(a), f(b))\}}{z-x} \leq \\
\leq \frac{2 f(z)-f(y)-\max \{f(a), f(b), g(f(a), f(b))\}}{z-y} .
\end{gathered}
$$

In connection with the preceding, a general convex function $f: D \rightarrow \mathbb{R}$ is said to be general convex with circled contact if (Max) holds, if there are $x_{0}, y_{0} \in D$ such that $f\left(x_{0}\right)=f\left(y_{0}\right)=g\left(f\left(x_{0}\right), f\left(y_{0}\right)\right)$, if $g(f(x), f(y)) \leq$ $g(f(x), f(z))$ for $x<y<z$, and if for $x_{1}<y_{1}<z_{1}$

$$
\min \left\{f\left(x_{1}\right), f\left(z_{1}\right), g\left(f\left(x_{1}\right), f\left(z_{1}\right)\right)\right\}<
$$

$<\max \left\{\max \left[f\left(x_{1}\right), f\left(z_{1}\right), g\left(f\left(x_{1}\right), f\left(z_{1}\right)\right)\right], \max \left[f\left(y_{1}\right), f\left(z_{1}\right), g\left(f\left(y_{1}\right), f\left(z_{1}\right)\right)\right]\right\}$

implies $(\mathrm{U})$ or $(\mathrm{V})$. Also, a function $f: D \rightarrow \mathbb{R}$ is said to be in circled contact with a function $g: f(D)^{2} \rightarrow \mathbb{R}$ if $f\left(x_{0}\right)=f\left(y_{0}\right)=g\left(f\left(x_{0}\right), f\left(y_{0}\right)\right)$ for some $x_{0}, y_{0} \in D$, if $g(f(x), f(y)) \leq g(f(x), f(z))$ for $x<y<z$, and if $(\mathrm{Mk})$ implies $(\mathrm{U})$ or $(\mathrm{V})$; if $g$ is a continuous function, then $f$ is said to be in continuous circled contact.

From the preceding, we are now in a position to formulate our the following characterizations for general convex functions.

Theorem 21. (Monotonity of quotients). Let $J \subset \mathbb{R}$ be an open interval, and let $f: J \rightarrow \mathbb{R}$ be a function in circled contact with a function $g: f(J)^{2} \rightarrow$ $\mathbb{R}$. Then each of the following conditions (postulated for every $x, y, z \in J$ with $x<y<z)$ is necessary and sufficient for the function $f$ to be general convex with circled contact $g$ :

$$
\begin{gathered}
f(y) \leq \max \{f(x), f(z), g(f(x), f(z))\}, \\
\frac{2 f(z)-f(x)-\max \{f(x), f(z), g(f(x), f(z))\}}{z-x} \leq \\
\leq \frac{2 f(z)-f(y)-\max \{f(y), f(z), g(f(y), f(z))\}}{z-y}, \\
\frac{2 f(y)-f(x)-\max \{f(x), f(y), g(f(x), f(y))\}}{y-x} \leq \\
\leq \frac{2 f(z)-f(x)-\max \{f(x), f(z), g(f(x), f(z))\}}{z-x} .
\end{gathered}
$$




\section{DifFERENTIATION OF GENERAL CONVEXITY}

Now let $J \subset \mathbb{R}$ be an open interval, and let $f: J \rightarrow \mathbb{R}$ and $g: f(J)^{2} \rightarrow \mathbb{R}$ be given functions. We define a function $g_{a, b}(I(x, h))$ for $x \in J$ and $h \in \mathbb{R}$ such that $h \neq 0, x+h \in J$ by

$$
\text { (Gp) } g_{p(a, b)}(I(x, h)):=\frac{f(x+h)-2 f(x)+\max \{f(a), f(b), g(f(a), f(b))\}}{h}
$$

and

$$
\text { (Gq) } g_{q(a, b)}(I(x, h)):=\frac{2 f(x+h)-f(x)-\max \{f(a), f(b), g(f(a), f(b))\}}{h}
$$

for arbitrary $a, b \in J$. For $a=x+h$ and $b=x$ in (Gp) and (Gq) we brief only $g_{p}(I(x, h))$ and $g_{q}(I(x, h))$, respectively. In this sense, we put that is

$$
g_{a, b}(I(x, h)):=\max \left\{g_{p(a, b)}(I(x, h)), g_{q(a, b)}(I(x, h))\right\},
$$

i.e.,

$$
g(I(x, h)):=\max \left\{g_{p}(I(x, h)), g_{q}(I(x, h))\right\}
$$

for $x \in J$ and $h \in \mathbb{R}$ such that $h \neq 0$ and $x+h \in J$.

According to Theorems 19, 20 and 21 if $f: J \rightarrow \mathbb{R}(J$ is an open interval) general convex with contact and continuous, the for every fixed $x \in J$ the differences quotient $g(I(x, h))$ is an increasing function of $h$. Consequently it has finite one-sided limits as $h$ tends to zero from the right, and from the left. But these limits are one-sided derivates of $f$ at $x$ :

$$
g\left(f_{+}^{\prime}(x)\right)=\lim _{h \rightarrow 0+} g(I(x, h)), \quad g\left(f_{-}^{\prime}(x)\right)=\lim _{h \rightarrow 0-} g(I(x, h)) .
$$

Thus we have the following result.

Theorem 22. Let $J \subset \mathbb{R}$ be an open interval, and let $f: J \rightarrow \mathbb{R}$ be continuous and general convex with continuous contact. Then at every point $x \in J$ there exists the right derivate $g\left(f_{+}^{\prime}(x)\right)$, and the left derivate $g\left(f_{-}^{\prime}(x)\right)$, and we have

$$
g\left(f_{-}^{\prime}(x)\right) \leq g\left(f_{+}^{\prime}(x)\right) \leq g\left(f_{-}^{\prime}(y)\right) \leq g\left(f_{+}^{\prime}(y)\right.
$$

for every $x, y \in J$ with $x<y$. Moreover,

$$
\begin{aligned}
& g\left(f_{+}^{\prime}(x)\right)=\lim _{t \rightarrow x+} g\left(f_{+}^{\prime}(t)\right)=\lim _{t \rightarrow x+} g\left(f_{-}^{\prime}(t)\right), \\
& g\left(f_{-}^{\prime}(x)\right)=\lim _{t \rightarrow x-} g\left(f_{+}^{\prime}(t)\right)=\lim _{t \rightarrow x-} g\left(f_{-}^{\prime}(t)\right) .
\end{aligned}
$$

The proof of this statement is an analogy with the proof in problem 23. Thus the proof we omit. For the proof of Theorem 22 see: Tas k ovi ć [2001].

Corollary 7. (Monotony of general derivates). Let $J \subset \mathbb{R}$ be an open interval, and let $f: J \rightarrow \mathbb{R}$ be a continuous general convex function with contunuous contact. Then the functions $g\left(f_{+}^{\prime}\right), g\left(f_{-}^{\prime}\right): J \rightarrow \mathbb{R}$ are increasing. 
Theorem 23. Let $J \subset \mathbb{R}$ be an open interval, and let $f: J \rightarrow \mathbb{R}$ continuous general convex function with continuous contact. The following three conditions are equivalent for every $x \in J$ :

(a) $g\left(f_{+}^{\prime}(x)\right)$ is a continuous function at $x$,

(b) $g\left(f_{-}^{\prime}(x)\right)$ is a continuous function at $x$,

(c) $f$ is general differentiable at $x$.

Proof. Condition (c) means that $g\left(f_{+}^{\prime}(x)\right)=g\left(f_{-}^{\prime}(x)\right)$, condition (a) that $\lim _{t \rightarrow x+} g\left(f_{+}^{\prime}(t)\right)=\lim _{t \rightarrow x-} g\left(f_{+}^{\prime}(t)\right)$, and condition (b) that $\lim _{t \rightarrow x+} g\left(f_{-}^{\prime}(t)\right)=$ $\lim _{t \rightarrow x-} g\left(f_{-}^{\prime}(t)\right)$. Relations (18) and (19) show that all the three conditions are equivalent. The proof is complete.

Theorem 24. Let $J \subset \mathbb{R}$ be an open interval, and let $f: J \rightarrow \mathbb{R}$ be a continuous general convex function with continuous contact. Then $f$ is general differentiable in $J$ except at at most countably many points. If $\subset J$ is the set of the points of the general differentiability of $f$, then the function $f^{\prime}: D \rightarrow \mathbb{R}$ is increasing and continuous.

Proof. The function $g\left(f_{+}^{\prime}(x)\right)$, being monotonic, may be discontinuous at at most countably many points. In view of Theorem 23 we obtain, hence the first part of our assertion. The second results from the fact that for $x \in D$, we have $g\left(f^{\prime}(x)\right)=g\left(f_{+}^{\prime}(x)\right)$ and by Theorem $23 g\left(f_{+}^{\prime}(x)\right)$ is contunuous at every point of $D$. The proof is complete.

In the sequel we say that $f$ is twice general differentiable function at a point $x_{0} \in J$ iff $x_{0} \in D$ (= the set of the points of general differentiability of $f$ ) and the limit of the form as

$$
g\left(f^{\prime \prime}\left(x_{0}\right)\right)=\left[g\left(f^{\prime}\left(x_{0}\right)\right)\right]^{\prime}:=\lim _{y \rightarrow x, y \in D} \frac{g\left(f^{\prime}(y)\right)-g\left(f^{\prime}\left(x_{0}\right)\right)}{y-x_{0}}
$$

exists, for every function $g: f(J)^{2} \rightarrow \mathbb{R}$ under which $f$ is a general convex function with continuous contact. Limit (DI) is equal to

$$
g\left(f^{\prime \prime}\left(x_{0}\right)\right)=\left[g\left(f^{\prime}\left(x_{0}\right)\right)\right]^{\prime}:=\lim _{y \rightarrow x, y \in D} \frac{g\left(f_{+}^{\prime}(y)\right)-g\left(f_{+}^{\prime}\left(x_{0}\right)\right)}{y-x_{0}}
$$

whenever the letter limit exists. But since the function $g\left(f_{+}^{\prime}(x)\right)$ is monotonic, it follows from the well-known Lebesgue's theorem that limit (DI') exists almost everywhere in $J$. Moreover, the general differences in (DI') is nonnegative (since $g\left(f_{+}^{\prime}(x)\right)$ is increasing), and consequently so is also $g\left(f^{\prime \prime}\left(x_{0}\right)\right)$ if it exists. Thus we obtain the following statement.

Theorem 25. Let $J \subset \mathbb{R}$ be an open interval, and let $f: J \rightarrow \mathbb{R}$ be a continuous general convex function with continuous contact. Then $f$ is twice general differentiable almost everywhere in $J$. Whenever it exists, $g\left(f^{\prime \prime}\left(x_{0}\right)\right) \geq 0$. 
We notice, since $g\left(f^{\prime}(x)\right)$ (defined almost everywhere in $J$ ) is monotonic, it is measurable, and bounded on every compact subinterval of $J$. Thus the expression

$$
\int_{a}^{x} g\left(f^{\prime}(t)\right) d t
$$

is meaningful for every $a, x \in J$. In this sense, we have the following statement in the form as:

Theorem 26. Let $J \subset \mathbb{R}$ be an open interval, and let $f: J \rightarrow \mathbb{R}$ be a continuous general convex function with continuous contact. Then, for all $a, x \in J$ we have the following expression

$$
f(x)=f(a)+\int_{a}^{x} g\left(f^{\prime}(t)\right) d t .
$$

Proof. Put $F(x)$ that is right side equality in (Gi). The function $F$ is general differentiable whenever $g\left(f^{\prime}\right)$ is continuous, and thus everywhere in $J$ with the exception of an at most countable set. If $x \in J$ is a point of the continuity of $g\left(f^{\prime}\right)$, then we have $F^{\prime}(x)=g\left(f^{\prime}(x)\right)$, i.e., $F-f=$ constant. Since at the point $a$ we have $F-f=0$, the constant must be zero, and thus $F=f$. Hence we obtain (Gi). The proof is complete.

As an immediate consequence of the preceding statement we obtain the following result in the well-known form as.

Theorem 27. Let $J \subset \mathbb{R}$ be an open interval, and let $f: J \rightarrow \mathbb{R}$ be a continuous general convex function with continuous contact. The function $f$ is absolutely continuous in $J$.

Annotation. The integral in (Gi) is Lebesgue integral, but everything remains unchanged if we replace this by the Riemann integral of $g\left(f_{+}^{\prime}(t)\right)$ !

General derivatives. Let $D \subset \mathbb{R}^{n}$ be an open set, and let $f: D \rightarrow \mathbb{R}$ be a function. We say that $f$ is general differentiable at a point $x \in D$ iff for every $y \in \mathbb{R}^{n}$ there exist the limits.

$g_{p}\left(f_{y}^{\prime}(x)\right):=\lim _{\lambda \rightarrow 0+} \frac{f(x+\lambda y)-2 f(x)+\max \{f(x), f(x+\lambda y), g(f(x), f(x+\lambda y))\}}{\lambda}$

and

$g_{q}\left(f_{y}^{\prime}(x)\right):=\lim _{\lambda \rightarrow 0+} \frac{2 f(x+\lambda y)-f(x)-\max \{f(x), f(x+\lambda y), g(f(x), f(x+\lambda y))\}}{\lambda}$

for a function $g: f(D)^{2} \rightarrow \mathbb{R}$, where the functions $g_{p}\left(f_{y}^{\prime}(x)\right)$ and $g_{q}\left(f_{y}^{\prime}(x)\right)$ are linear of $y \in \mathbb{R}^{n}$.

Precisely, let $D \subset \mathbb{R}^{n}$ be an open set, and let $f: D \rightarrow \mathbb{R}$ be a function. We say that $f$ is general differentiable at a point $x \in D$ iff for every $y \in \mathbb{R}^{n}$ there exists the limit

$$
g\left(f_{y}^{\prime}(x)\right)=\max \left\{g_{p}\left(f_{y}^{\prime}(x)\right), g_{q}\left(f_{y}^{\prime}(x)\right)\right\}
$$


for a function $g: f(D)^{2} \rightarrow \mathbb{R}$, where the function $g\left(f_{y}^{\prime}(x)\right)$ is a linear function of the point $y \in \mathbb{R}^{n}$.

Theorem 28. Let $D \subset \mathbb{R}^{n}$ be an open and convex set, and let $f: D \rightarrow \mathbb{R}$ be a continuous general convex function with continuous contact under the function $g: f(D)^{2} \rightarrow \mathbb{R}$ such that $g(t, t)=t$ for $t \in f(D)$. At every point $x \in D$ the general derivative $g\left(f_{y}^{\prime}(x)\right)$ exists for every $y \in \mathbb{R}^{n}$. Moreover, $g\left(f_{y}^{\prime}(x)\right)$ is a general convex, continuous and positively homogeneous function and, for arbitrary $\mu<0$ the following inequality holds

$$
-g\left(f_{|\mu| y}^{\prime}(x)\right) \leq g\left(f_{\mu y}^{\prime}(x)\right) \quad \text { for all } y \in \mathbb{R}^{n} .
$$

\section{Representing of General CONVEXity}

Further, we give representing a general convex function as an integral inequality which, fortunately, can be taken in the sense of either Riemann or Lebesgue.

Theorem 29. Let $f: J \rightarrow \mathbb{R}(J \subset \mathbb{R}$ is an open interval) be a continuous function. Then $f$ is general convex with continuous contact if and only if there is an increasing (strictly increasing) function $\psi: J \rightarrow \mathbb{R}$ and a point $\rho \in J$ such that

$$
f(x)-f(\rho) \leq \frac{1}{2} \int_{\rho}^{x} \psi(t) d t \quad \text { for every } \quad x \in J .
$$

Proof. We suppose firs that $f$ is general convex function with continuous contact over a bisection function $g: f(J)^{2} \rightarrow \mathbb{R}$. By Theorem 22 we obtain the following inequalities

$$
\begin{gathered}
\frac{f\left(x_{k}\right)-2 f\left(x_{k-1}\right)+}{\max \left\{f\left(x_{k}\right), f\left(x_{k-1}\right), g\left(f\left(x_{k}\right), f\left(x_{k-1}\right)\right)\right\}} \leq \\
\left.x_{k}-x_{k-1}\right) \\
\leq g\left(f_{-}^{\prime}\left(x_{k}\right)\right) \leq g\left(f_{+}^{\prime}\left(x_{k}\right)\right)
\end{gathered}
$$

where $\rho=x_{0}<x_{1}<\cdots<x_{n}=x$ is a partition of $[\rho, x]$ for every $k \in$ $\{1, \ldots, n\}$. Since by elementary the following inequality holds in the form

$2\left(f\left(x_{k}\right)-f\left(x_{k-1}\right)\right) \leq f\left(x_{k}\right)-2 f\left(x_{k-1}\right)+\max \left\{f\left(x_{k}\right), f\left(x_{k-1}\right), g\left(f\left(x_{k}\right), f\left(x_{k-1}\right)\right)\right\}$

hence, with addition of left and right sides, we obtain the following inequalities in the forms as

$$
2\left(f\left(x_{k}\right)-f(\rho)\right) \leq \sum_{k=1}^{n} g\left(f_{-}^{\prime}\left(x_{k}\right)\right)\left(x_{k}-x_{k-1}\right) \leq \sum_{k=1}^{n} g\left(f_{+}^{\prime}\left(x_{k}\right)\right)\left(x_{k}-x_{k-1}\right) .
$$

thus, letting in the preceding inequalities that $n \rightarrow \infty$, we obtain the following inequalities in the form as

$$
2(f(x)-f(\rho)) \leq \int_{\rho}^{x} g\left(f_{-}^{\prime}(t)\right) d t \leq \int_{\rho}^{x} g\left(f_{+}^{\prime}(t)\right) d t
$$


Since the functions $g\left(f_{+}^{\prime}(x)\right)$ and $g\left(f_{-}^{\prime}(x)\right)$ are increasing, $\psi=g\left(f_{-}^{\prime}(x)\right)$ or $\psi=g\left(f_{+}^{\prime}(x)\right)$ will be increasing. Thus the inequality $(\mathrm{N})$ holds.

Conversely, suppose that inequality $(\mathrm{N})$ holds with an increasing function $\psi: J \rightarrow \mathbb{R}$. Then for all $x, y \in J(x<y)$ it follows

$$
(\alpha+\beta) f(\alpha x+\beta y)-\alpha f(x)-\beta f(y) \leq \frac{\alpha}{2} \int_{x}^{\alpha x+\beta y} \psi(t) d t-\frac{\beta}{2} \int_{\alpha x+\beta y}^{y} \psi(t) d t,
$$

where $\alpha+\beta=1(\alpha, \beta>0)$. To bound this expression below, we replace both integrands by the constant $\psi(\alpha x+\beta y)$, this baing the smallest value of the first integrand and the largest of the second. We obtain on the right-hand side

$$
\frac{\alpha}{2} \psi(\alpha x+\beta y)(\alpha x+\beta y-x)-\frac{\beta}{2} \psi(\alpha x+\beta y)[y-(\alpha x+\beta y)]=0 .
$$

hence, it follows the following inequality for an arbitrary function $g: f(J)^{2} \rightarrow$ $\mathbb{R}$ in the form as

$f(\alpha x+\beta y)-\max \{f(x), f(y), g(f(x), f(y))\} \leq f(\alpha x+\beta y)-\alpha f(x)-\beta f(y)$ which is equivalent to the inequality that definies general convexity with continuous contact. The proof is comeplete.

Proposition 8 (Characterization of convex functions). The function $f$ : $J \rightarrow \mathbb{R}$ (where $J$ is an open interval) is convex if and only if there is an increasing (strictly increasing) function $\psi: J \rightarrow \mathbb{R}$ and a point $\rho \in J$ such that

$$
f(x)-f(\rho)=\int_{\rho}^{x} \psi(t) d t \quad \text { for every } \quad x \in J .
$$

Proof. We suppose first that $f$ is convex. Choose $\psi=f_{+}^{\prime}$ which exists and is increasing and let $\rho$ be a point in $J$. By problem 26, $f$ is absolutely continuous on $J$. By an elementary argument for Riemann integrals or by a classical theorem for Lebesgue integrals in Natanson [1961, p. 255] we obtain

$$
f(x)-f(\rho)=\int_{\rho}^{x} f_{+}^{\prime}(t) d t=\int_{\rho}^{x} \psi(t) d t,
$$

moreover, if $f$ is strictly convex, $\psi=f_{+}^{\prime}$ will be strictly increasing. For conversely the proof is totally analogous with the proof in the preceding result.

Annotation. An elementay argument involving only the concepts of the Riemann integral can be used to show that if $f$ is convex on $J$, then for all $\rho, x \in J$ we obtain the following equalities in the form as

$$
f(x)-f(\rho)=\int_{\rho}^{x} f_{-}^{\prime}(t) d t=\int_{\rho}^{x} f_{+}^{\prime}(t) d t,
$$


where $\rho=x_{0}<x_{1}<\cdots<x_{n}=x$ is a partition of $[\rho, x]$ and where for all $k \in\{1, \ldots, n\}$. we have the following inequalities of the form as

$$
f_{-}^{\prime}\left(x_{k-1}\right) \leq f_{+}^{\prime}\left(x_{k-1}\right) \leq \frac{f\left(x_{k}\right)-f\left(x_{k-1}\right)}{x_{k}-x_{k-1}} \leq f_{-}^{\prime}\left(x_{k}\right) \leq f_{+}^{\prime}\left(x_{k}\right) .
$$

Thus, we can obtain the second proof of the equality (Ko), i.e., we can obtain the second proof of Proposition 3.

In connection with this result, as an analogous, we obtain directly the following statement.

Theorem 29a. Let $f: J \rightarrow \mathbb{R}(J \subset \mathbb{R}$ is an open interval $)$ be a continuous function. Then $f$ is general convex with continuous contact if and only if there is a convex function $K: J \rightarrow \mathbb{R}$ and a point $\rho \in J$ such that

$$
f(x)-f(\rho) \leq K(x)-K(\rho) \quad \text { for every } \quad x \in J .
$$

Proof. Suppose that $f$ is a general convex function with continuous contact. Thus, from Theorem 29, it follows that inequality (N) hods. Choose,

$$
K(x)=2 \int_{\rho}^{x} \psi(t) d t \quad \text { for } \quad x \in J
$$

for an increasing function $\psi: J \rightarrow \mathbb{R}$ and some $\rho \in J$, we obtain directly that inequality $(\mathrm{K})$ holds.

Conversely, if inequality $(\mathrm{K})$ holds, then from Proposition 3 and Theorem 29 it follows that $f$ is a general convex function with continuous contact. The proof is complete.

Differences of convex and general convex functions. The class of all convex functions on an interval $J$ is closed under addition, but it is not closed under scalar multiplication or subtraction. We may, of course, consider the class of functions representable as the difference of convex and general convex functions.

Let $B K(J)$ for $J:=[a, b]$ be the class of functions $f: J \rightarrow \mathbb{R}$ representable in the form as $f \leq \zeta-h$, where $\zeta$ is a continuous general convex function with continuous contact, $h$ is a convex function, such that $g\left(\zeta_{+}^{\prime}(a)\right), g\left(\zeta_{-}^{\prime}(b)\right)$, $h_{+}^{\prime}(a)$, and $h_{-}^{\prime}(b)$ are all finite.

Then $B K(J)$ is a linear space. Moreover, it is easily characterized in terms of $B V(J)$, the space of functions of bounded variation.

Proposition 9. The function $f: J \rightarrow \mathbb{R}(J:=[a, b] \subset \mathbb{R})$ is in $B K(J)$ if and only if there exists a function of bounded variation $r: J \rightarrow \mathbb{R}$ such that

$$
f(x)-f(a) \leq \frac{1}{2} \int_{a}^{x} r(t) d t \quad \text { for every } \quad x \in J .
$$


Proof. If $f \in B K(J)$, then $f \leq \zeta-h$, where $\zeta$ is general convex function with continuous contact and $h$ is a convex function. Also, $\zeta$ and $h$ have finite endpoint derivatives, By Theorem 29 and Proposition 3 we obtain

$$
\zeta(x)-\zeta(a) \leq \frac{1}{2} \int_{a}^{x} p(t) d t, \quad h(x)-h(a)=\int_{a}^{x} q(t) d t
$$

for some increasing functions $p, q: J \rightarrow \mathbb{R}$. Thus, directly computation and majorization, we obtain the following two inequalities in the form as

$$
f(x)-f(a) \leq(\zeta(x)-\zeta(a))-(h(x)-h(a)) \leq \frac{1}{2} \int_{a}^{x}[p(t)-2 q(t)] d t,
$$

where $p-2 q$ is a function of bounded variation as differences of two increasing functions.

Conversely, if $f$ satisfies $(\mathrm{R})$, then the fundamental characterization of functions of bounded variation enables us to write $r(t)=p(t)-q(t)$, where $p$ and $q$ are increasing on $J$ give

$$
f(x) \leq f(a)+\int_{a}^{x} p(t) d t-\int_{a}^{x} q(t) d t,
$$

and it is therefore the difference of a convex function and a general convex function with continuous contact. The endpoint conditions are easily established. The proof is complete.

We may, of course, consider the class of functions representable as the difference of two convex functions.

Proposition 10. The function $f: J \rightarrow \mathbb{R}(J:=[a, b] \subset \mathbb{R})$ is in the form $f=\zeta-h$, where $\zeta$ and $h$ are convex functions with finitess of $\zeta_{+}^{\prime}(a), \zeta_{-}^{\prime}(b)$, $h_{+}^{\prime}(a)$ and $h_{-}^{\prime}(b)$, if and only if there exists a function of bounded variation $r: J \rightarrow \mathbb{R}$ such that

$$
f(x)-f(a)=\int_{a}^{x} r(t) d t \quad \text { for every } \quad x \in J .
$$

The proof of this statement is totally analogy with the preceding proof of Proposition 4. Thus the proof we omit. For further facts see: R o b e r t s-V a r b e r g [1973], N a t a nson [1961] and Tasković [2001].

\section{INEQUALITIES OF GENERAL CONVEXITY}

In this section we continue the study and considerations of the general convex functions, which are introduced by Tas k ovi ć: Math. Japonica, 37(1992), 367-372.

We begin with introduce a concept of general subgradient for general convex functions. Subgradients generalize the classical concept of a derivate. In this connection, for our concept of general subgradient, we prove existence statement. Every general convex with tent function have the nontrivial (different of zero) general subgradient. In this sense further we consider general subgradient and general subdifferential of general convex functions and their further applications.

Since the general convex functions are defined by a functional inequality, it is not surprising that this notion will lead to a number of fundamental inequalities. Finally, 
applications of the preceding statements we give some fundamental inequalities for general convex functions.

The following main result is proved: Let $J \subset \mathbb{R}$ be an open interval, let $x_{i}, y_{i} \in J$ $(i=1, \ldots, n)$ be real numbers such that fulfilling $x_{1} \geq \cdots \geq x_{n}, y_{1} \geq \cdots \geq y_{n}$. Then, a necessary and sufficient condition in order that

$$
\sum_{i=1}^{n} f\left(x_{i}\right) \geq 2 \sum_{i=1}^{n} f\left(y_{i}\right)-n \max \{f(a), f(b), g(f(a), f(b))\}
$$

holds for every general convex function $f: J \rightarrow \mathbb{R}$ which is in contact with function $g: f(J)^{2} \rightarrow \mathbb{R}$ and for arbitrary $a, b \in J\left(a \leq x_{i} \leq b\right.$ for $\left.i=1, \ldots, n\right)$, is that

$$
\sum_{i=1}^{k} y_{i} \leq \sum_{i=1}^{k} x_{i} \quad(k=1, \ldots, n-1), \quad \sum_{i=1}^{n} y_{i}=\sum_{i=1}^{n} x_{i} .
$$

8.1. Introduction and history. This section continues the study of the general convex functions. A function $f: D \rightarrow \mathbb{R}$, where $\mathbb{R}$ denotes the real line and $D$ is a convex subset of $\mathbb{R}^{n}$, is said to be convex if

$$
f(\lambda x+(1-\lambda) y) \leq \lambda f(x)+(1-\lambda) f(y)
$$

for all $x, y \in D$ and for arbitrary $\lambda \in[0,1]$. Convex functions were introduced (for $n=1$ and $\lambda=1 / 2$ ) by J. L. Jensen in 1906, although functions satisfying similar conditions were already treated by O. Hölder, J. Hadamard, Ch. Hermite, and O. Stolz.

Theorem 30. Let $D \subset \mathbb{R}^{n}$ be a convex and open set. If $f: D \rightarrow \mathbb{R}$ is a general convex function, then there is a function $g: f(D)^{n} \rightarrow \mathbb{R}$ such that

$$
f\left(\frac{\sum_{i=1}^{n} p_{i} x_{i}}{\sum_{i=1}^{n} p_{i}}\right) \leq \max \left\{f\left(x_{1}\right), \ldots, f\left(x_{n}\right), g\left(f\left(x_{1}\right), \ldots, f\left(x_{n}\right)\right)\right\}
$$

for every $n \in \mathbb{N}$, for all points $x_{1}, \ldots, x_{n} \in D$, and for arbitrary numbers $p_{1}, \ldots, p_{n} \in \mathbb{R}_{+}^{0}$ such that $p_{1}+\cdots+p_{n}>0$.

Proof. The preceding inequality (Tn) directly is consequence of Theorem 2 in the following sense. If in inequality (DN') put $\lambda_{i}=p_{i} /\left(p_{1}+\cdots+p_{n}\right)$, we obtain (Tn). The proof is complete.

Theorem 31. Let $D \subset \mathbb{R}^{n}$ be a convex and open set and let there exist numbers $p_{i} \in \mathbb{R}(i=1, \ldots, n)$ and $k \in\{1, \ldots, n\}$ such that

$$
p_{k}>0, \quad p_{i} \leq 0 \quad(i \neq k), \quad p_{1}+\cdots+p_{n}>0,
$$

where $\xi:=\left(p_{1}+\cdots+p_{n}\right)^{-1}\left(p_{1} x_{1}+\cdots+p_{n} x_{n}\right) \in D$ for all $x_{1}, \ldots, x_{n} \in$ $D$. If $f: D \rightarrow \mathbb{R}$ is a general convex function, then there is a function $g: f(D)^{n} \rightarrow \mathbb{R}$ such that

$$
\begin{gathered}
f\left(\frac{\sum_{i=1}^{n} p_{i} x_{i}}{\sum_{i=1}^{n} p_{i}}\right) \leq \max \left\{f\left(x_{1}\right), \ldots, f\left(x_{k-1}\right), f(\xi), f\left(x_{k+1}\right), \ldots\right. \\
\left.\quad \ldots, f\left(x_{n}\right), g\left(f\left(x_{1}\right), \ldots, f\left(x_{k-1}\right), f(\xi), f\left(x_{k+1}\right), \ldots, f\left(x_{n}\right)\right)\right\}
\end{gathered}
$$


for every $n \in \mathbb{N}$, for all points $x_{1}, \ldots, x_{n} \in D$, and for arbitrary numbers $p_{1}, \ldots, p_{n} \in \mathbb{R}$ with the property (21).

Proof. If to exchange $p_{k}$ with $p_{1}+\cdots+p_{n}, x_{k} \in D$ with $\left(p_{1}+\cdots+\right.$ $\left.p_{n}\right)^{-1}\left(p_{1} x_{1}+\cdots+p_{n} x_{n}\right) \in D$, the points $x_{i}(i \neq k)$ with the points $x_{i}$ $(i \neq k)$, and $p_{i}$ with $-p_{i}$ in Theorem 30, we obtain inequality (Tn) and thus calculation it follows inequality $(\mathrm{Tr})$. The proof is complete.

Annotation. In connection with inequality ( $\mathrm{Tn})$ we have the following fact. Let $D \subset \mathbb{R}^{n}$ be a convex and open set. If $f: D \rightarrow \mathbb{R}$ is a general convex function, then there is a function $g\left(f(D)^{n} \rightarrow \mathbb{R}\right.$ such that

$\operatorname{Sup} \underset{\left(p_{1}+\cdots, p_{n}>0\right)}{p_{1}, \ldots, p_{n} \in \mathbb{R}} f\left(\frac{\sum_{i=1}^{n} p_{i} x_{i}}{\sum_{i=1}^{n} p_{i}}\right) \leq \max \left\{f\left(x_{1}\right), \ldots, f\left(x_{n}\right), g\left(f\left(x_{1}\right), \ldots, f\left(x_{n}\right)\right)\right\}$

for every $n \in \mathbb{N}$, for all points $x_{1}, \ldots, x_{n} \in D$, and for arbitrary numbers $p_{1}, \ldots, p_{n} \in \mathbb{R}_{+}^{0}$ such that $p_{1}+\cdots+p_{n}>0$

Equality holds in inequality (Ts) if and only if $f(x)=g(f(x), \ldots, f(x))$ for every point $x \in D$.

In connection with the preceding facts, let $D \subset \mathbb{R}^{n}$ be a convex and open set. The function $f: D \rightarrow \mathbb{R}$ is a general $\psi$-convex function if there exist a function $\psi: \mathbb{R}_{+}^{0} \rightarrow \mathbb{R}_{+}^{0}$ with $\psi(0)=0$ and a function $g: f(D)^{2} \rightarrow \mathbb{R}$ such that

$f(\lambda x+(1-\lambda) y) \leq \max \{f(x), f(y), g(f(x), f(y))\}+\max \{\lambda, 1-\lambda\} \psi(\|x-y\|)$

for all $x, y \in D$ and for arbitrary $\lambda \in[0,1]$. Applying Proposition 10 analogous to the preceding facts we obtan the following result.

Theorem 32. (Tasković, [1994]). Let $D \subset \mathbb{R}^{n}$ be a convex and open set. If $f: D \rightarrow \mathbb{R}$ is a general $\psi$-convex function, then there is a function $g$ : $f(D)^{n} \rightarrow \mathbb{R}$ such that

$$
\begin{gathered}
f\left(\frac{\sum_{i=1}^{n} p_{i} x_{i}}{\sum_{i=1}^{n} p_{i}}\right) \leq \max \left\{f\left(x_{1}\right), \ldots, f\left(x_{n}\right), g\left(f\left(x_{1}\right), \ldots, f\left(x_{n}\right)\right)\right\}+ \\
+\frac{1}{p_{1}+\cdots+p_{n}} \sum_{i<j \leq n} \max \left\{p_{i}, p_{j}\right\} \psi\left(\left\|x_{i}-x_{j}\right\|\right),
\end{gathered}
$$

for every $n \in \mathbb{N}$, for all points $x_{1}, \ldots, x_{n} \in D$, and for arbitrary numbers $p_{1}, \ldots, p_{n} \in \mathbb{R}_{+}^{0}$ with the property $p_{1}+\cdots+p_{n}>0$.

In the sequel we will need one fundamental notion. Let $D \subset \mathbb{R}^{n}$ be an open set, and let $f: D \rightarrow \mathbb{R}$ be a function. Any $x^{*} \in \mathbb{R}^{n}$ such that

$$
f(z) \geq f(x)+x^{*}(z-x) \text { for } z \in D
$$

is called a subgradient of $f$ at $x$. The set of all subgradients of $f$ at $x$ will be denoted by $\partial f(x)$. Of course, it may happen that $\partial f(x)=\varnothing$. But, if 
$f: D \rightarrow \mathbb{R}$ be a convex function, then (C) holds, i.e., $\partial f(x)$ is a nonempty set for every $x \in D$.

In this section we begin with a concept of general subgradient for general convex functions. For the case of convex functions see: Figure 6.

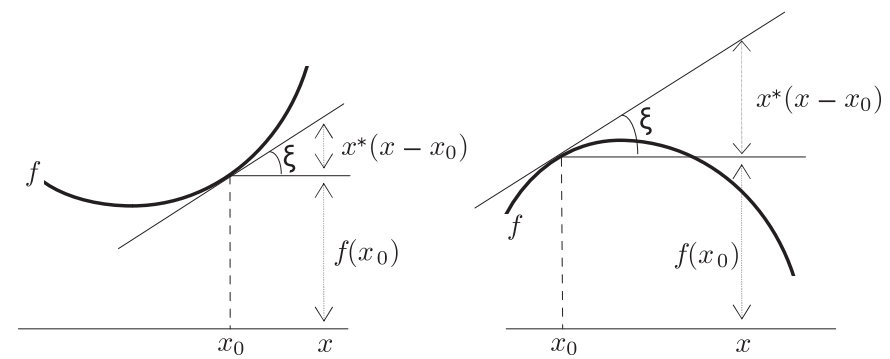

FiguRE 6

Subgradient generalize the classical concept of a derivate. In connection with this, in this section we consider a concept of general subgradient for general convex functions.

Let $D \subset \mathbb{R}^{n}$ be an open set and let $f: D \rightarrow \mathbb{R}$ be a function. Any $x^{*} \in \mathbb{R}^{n}$ is said to be general subgradient if there is a function $g: f(D)^{2} \rightarrow \mathbb{R}$ such that (GS):

$|f(z)-f(x)| \geq f(\lambda a+(1-\lambda) b)+x^{*}(z-x)-\max \{f(a), f(b), g(f(a), f(b))\}$

for $z \in D$ and $\lambda \in[0,1]$, where $a, b \in D$ denoted in further two arbitrary points. The point $x^{*}$ is called a general subgradient of $f$ at $x$.

The set of all general subgradients of $f$ at $x$ will be denoted by $g(\partial f(x))$. If no general subgradient exist at $x$, then we set $g(\partial f(x))=\varnothing$.

We notice, if $f$ is general convex, then (Max) and so (GS) is satisfied with $x^{*}=0$. Thus $x^{*}=0$ is a trivial general subgradient of every general convex function at every point $x \in D$. This means that are of importance only nontrivial $(:=$ different of zero) general subgradients.

The set $g(\partial f(x))$ is called a nontrivial if there is a general subgradient $x^{*} \neq 0$ of $f \not \equiv 0$ at point $x \in D$.

Simple see, if (GS) holds for all $x \in D$, then (GS) means that $f$ is a general convex function on $D$. We notice, also, simple will see that if function $f: D \rightarrow \mathbb{R}$ is the general convex, then the following inequality holds

$\left(\mathrm{Max}^{\prime}\right) \quad f(x) \leq \max \{f(a), f(b), g(f(a), f(b))\} \quad$ for all $\quad x \in[a, b] \subset D$

for some function $g: f(D)^{2} \rightarrow \mathbb{R}$ and for two arbitrary points $a, b \in D(a<$ $b)$, where $[a, b]$ denoted an interval in $D$. This fact has further interesting consequences.

In this paper further we consider general subgradient, general subdifferential of general convex functions and their further applications for some fundamental inequalities. 
In this sense, a function $f: D \rightarrow \mathbb{R}\left(D \subset \mathbb{R}^{n}\right.$ is a convex subset) is said to be general convex with tent if there is a function $g: f(D)^{2} \rightarrow \mathbb{R}$ such that (Max) and that

$$
S:=\left\{(x, y) \in \mathbb{R}^{n+1}: x \in D, y \geq \max \{f(a), f(b), g(f(a), f(b))\}\right\}
$$

is a convex set for arbitrary $a, b \in D$.

8.2. Existence of general subgradients. Subgradient generalize the classical concept of a derivate. In this section we will be concerned concept of generalize sugradient for general convex functions and their existence.

Theorem 33. Let $D \subset \mathbb{R}^{n}$ be a convex and open set, and let $f: D \rightarrow \mathbb{R}$ be a general convex with tent function. Then $g(\partial f(x))$ is a nonempty nontrivial set for every $x \in D$.

Proof. Let $a, b \in D$ be two arbitrary points. Also, let $g: f(D)^{2} \rightarrow \mathbb{R}$ be a function for which $f$ is a general convex with tent function. Consider the set

$$
S=\left\{(x, y) \in \mathbb{R}^{n+1}: x \in D, y>\max \{f(a), f(b), g(f(a), f(b))\}\right\} .
$$

Since $f$ is a general convex with tent function the set $S$ is open and convex and the points $(x, \max \{f(a), f(b), g(f(a), f(b))\}) \in \mathbb{R}^{n+1}$ are its frontier points. Well known, that if $S \subset \mathbb{R}^{n}$ is a convex set, then through every point of the frontier of $S$ there passes a support hyperplane of $S$ (see Mazur in 1933). According to this Mazur's theorem there exists a support hyperplane $H$ of $S$ passing through the point of frontier $(x, \max \{f(a), f(b), g(f(a), f(b))\})$. The hyperplane $H$ has an equation of the form

$$
c(z-x)+\alpha(y-\max \{f(a), f(b), g(f(a), f(b))\})=0,
$$

where $c \in \mathbb{R}^{n}, \alpha \in \mathbb{R}$, and $(z, y)$ is the current point of $\mathbb{R}^{n+1}$. If we had $\alpha=0$, then every point $(x, y)$ with an arbitrary $y \in \mathbb{R}$, would satisfy (22), and taking $y>\max \{f(a), f(b), g(f(a), f(b))\}$ we would get that $H \cap S$ is nonempty, and so $S$, being open, would have points on both sides of $H$, which is impossible.

Consequently $\alpha \neq 0$. So we may write (22) in the form $y=\max \{f(a), f(b)$, $g(f(a), f(b))\}-c \alpha^{-1}(z-x)$. The two halfspaces into which $H$ divides $\mathbb{R}^{n+1}$ are determined by the inequalities

$$
y>\max -c \alpha^{-1}(z-x) \text { and } y<\max -c \alpha^{-1}(z-x),
$$

where $\max :=\max \{f(a), f(b), g(f(a), f(b))\}$. For arbitrary $y>\max$ the point $(x, y) \in S$ and clearly we have $y>\max -c \alpha^{-1}(x-x)$ so that the set $S$ must be contained in the halfspace determined by the inequality

$$
y>\max \{f(a), f(b), g(f(a), f(b))\}-c \alpha^{-1}(z-x) .
$$


On the other hand, since $f$ is a general convex function, we obtain that holds inequality (Max), i.e., inequality $\max -f(\xi) \geq 0$, for $\xi:=\lambda a+(1-$ $\lambda) b$ and $\lambda \in[0,1]$. Thus holds and inequality of the form $|f(z)-f(x)|+$ $\max -f(\xi) \geq 0$, i.e., holds inequality $|f(z)-f(x)|+2 \max -f(\xi) \geq \max$. Take an arbitrary $z \in D$ and an arbitrary $t>|f(z)-f(x)|+2 \max -f(\xi)$. Then $(z, t) \in S$, whence $t>\max -c \alpha^{-1}(z-x)$. Letting

$$
t \rightarrow|f(z)-f(x)|-f(\xi)+2 \max \{f(a), f(b), g(f(a), f(b))\},
$$

we obtain hence the following inequality

$$
|f(z)-f(x)| \geq f(\xi)-c \alpha^{-1}(z-x)-\max \{f(a), f(b), g(f(a), f(b))\},
$$

and this is valid for all $z \in D$, which shows that $-c \alpha^{-1} \in g(\partial f(x))$, and thus $g(\partial f(x))$ is a nontrivial nonempty set. The proof is complete.

As an immediate analogous of the preceding Theorem 33, we are now in a position to formulate the following statement.

Theorem 33a. Let $D \subset \mathbb{R}^{n}$ be a convex and open set, and let $f: D \rightarrow \mathbb{R}$ be a general convex with tent function for some function $g: f(D)^{2} \rightarrow \mathbb{R}$. Then for every $x \in D$ there is a point $x^{*} \in \mathbb{R}^{n}\left(x^{*} \neq 0\right)$ such that

$$
|f(z)| \geq f(\lambda a+(1-\lambda) b)+x^{*}(z-x)-\max \{f(a), f(b), g(f(a), f(b))\}
$$

for $z \in D, \lambda \in[0,1]$ and for two arbitrary points $a, b \in D$. Also, for every $x \in D$ there is a point $x^{*} \in \mathbb{R}^{n}\left(x^{*} \neq 0\right)$ such that the following inequality holds $(\mathrm{Ga})$ :

$$
|| f(z)|-| f(x)|| \geq f(\lambda a+(1-\lambda) b)+x^{*}(z-x)-\max \{f(a), f(b), g(f(a), f(b))\}
$$

for $z \in D, \lambda \in[0,1]$ and for two arbitrary points $a, b \in D$.

The proof of this statement is analogous to the proof of the preceding statement of Theorem 33.

In connection with the preceding, since the glass of all convex function is a proper subclass of the set all general convex with tent functions, we obtain directly the following well-known result in 1963 for convex functions.

Theorem 33b. (Bishop-Phelps, [1963]). Let $D \subset \mathbb{R}^{n}$ be a convex and open set, and let $f: D \rightarrow \mathbb{R}$ be a convex function. Then for every $x \in D$ there is $x^{*} \in \mathbb{R}^{n}$ such that

$$
f(z) \geq f(x)+x^{*}(z-x) \quad \text { for } \quad z \in D .
$$

Proof. If to teasing on the convex class functions taking $g(f(a), f(b))=$ $\lambda f(a)+(1-\lambda) f(b)$ for arbitrary $\lambda \in[0,1]$ and $a=b=x$, then we obtain that the former set

$$
S=\left\{(x, y) \in \mathbb{R}^{n+1}: x \in D, \quad y>f(x)\right\}
$$

is a convex set. Thus $f$ is general convex with tent. Applying Theorem 33 to this case we obtain that (GS) holds for every $x \in D$. Since $f$ has only a lower line of suppport, from (GS) we have (Ca):

$f(z) \geq f(x)+f(\lambda a+(1-\lambda) b)+x^{*}(z-x)-\max \{(f(a), f(b), g(f(a), f(b))\}$. 
Taking $a=b=x$ and $g(f(a), f(b))=\lambda f(a)+(1-\lambda) f(b)$ for arbitrary $\lambda \in[0,1]$ in $(\mathrm{Ca})$ we obtain $f(z) \geq 2 f(x)+x^{*}(z-x)-\lambda f(z)-(1-\lambda) f(x)$ for $z \in D$, which means that (C) holds. The proof is complete.

8.3. Derivates of general convex functions. Let $D \subset \mathbb{R}^{n}$ be an open set, and let $f: D \rightarrow \mathbb{R}$ be a function. We say that $f$ is left general differentiable at a point $x \in D$ if there is function $g: f(D)^{2} \rightarrow \mathbb{R}$ such that for every $y \in \mathbb{R}^{n}$ there exists the following limit

(GD)

$$
g_{p(a, b)}\left(f_{y}^{\prime}(x)\right):=\lim _{\lambda \rightarrow 0+} \frac{f(x+\lambda y)-2 f(x)+\max \{f(a), f(b), g(f(a), f(b))\}}{\lambda}
$$

for arbitrary $a, b \in D$, where $g_{p(a, b)}\left(f_{y}^{\prime}(x)\right)$ is a linear function of $y$. It follows from (GD) that we always have $g_{p(a, b)}\left(f_{0}^{\prime}(x)\right)=0$, where $a=b=x$ and $g(f(x), f(x))=f(x)$.

On the other hand, so we introduce one more notion. Let $D \subset \mathbb{R}^{n}$ be an open set, and let $f: D \rightarrow \mathbb{R}$ be a function. We say that $f$ has a left general differential at the point $x \in D$ if there function $g: f(D)^{2} \rightarrow \mathbb{R}$ and if there exists an $x^{*} \in \mathbb{R}^{n}$ such that

(GD')

$$
f(z)=2 f(x)+x^{*}(z-x)-\max \{f(x), f(z), g(f(x), f(z))\}+r(x, z)
$$

for $z \in D$, where the function $r: \mathbb{R}^{2 n} \rightarrow \mathbb{R}$ fulfils the following condition in the form

$$
\lim _{z \rightarrow x} \frac{r(x, z)}{\|z-x\|}=0 .
$$

Of course, every function $f$ can be written in form (GD') with every $x^{*} \in \mathbb{R}^{n}$. It is enough to put $r(x, z)=f(z)-2 f(x)-x^{*}(z-x)+$ $\max \{f(x), f(z), g(f(x), f(z))\}$. Here the problem lies in that $x^{*}$ should be chosen in such a manner that the coresponding function $r$ should satisfy (Gr).

Theorem 34. Let $D \subset \mathbb{R}^{n}$ be a convex and open set, and let $f: D \rightarrow \mathbb{R}$ be a general convex with contact function for some function $g: f(D)^{2} \rightarrow \mathbb{R}$. Then for every $x^{*} \in \mathbb{R}^{n}$ we have

$$
g_{p(a, b)}\left(f_{y}^{\prime}(x)\right) \geq x^{*} y \quad \text { for all } y \in \mathbb{R}^{n}
$$

if and only if the following inequality holds

$$
f(z) \geq 2 f(x)+x^{*}(z-x)-\max \{f(a), f(b), g(f(a), f(b))\}
$$

for $z \in D$ and for two arbitrary points $a, b \in D$.

Proof. First we note, by the definition of (23') if fix a $\lambda>0$ and put $y=$ $\lambda^{-1}(z-x)$, then (23') goes into

$$
f(x+\lambda y) \geq 2 f(x)+x^{*} \lambda y-\max \{f(a), f(b), g(f(a), f(b))\} .
$$


Conversely (23') results from (23e) on putting $z=x+\lambda y$. Thus (23') is equivalent with (23e). On the other hand, if (23'), i.e., (23e) holds, then we have

$$
F(\lambda):=\frac{f(x+\lambda y)-2 f(x)+\max \{f(a), f(b), g(f(a), f(b))\}}{\lambda} \geq x^{*} y
$$

for all $y \in \mathbb{R}^{n}$ and $\lambda>0$ such that $x+\lambda y \in D$, whence on passing to the limit as $\lambda \rightarrow 0+$, we obtain (23).

Also, let (23) holds. Since $f$ is general convex with contact, we have from Theorem 20 that $F(\lambda)$ is an increasing function for $\lambda>0$, and thus we obtain the following inequalities

$$
F(\lambda) \geq g_{p(a, b)}\left(f_{y}^{\prime}(x)\right) \geq x^{*} y .
$$

Hence we obtain (23e), which means that (23') holds. Now the proof is complete.

As an immediate consequence of the preceding proof of Theorem 34, we are now in a position to formulate the following statement.

Theorem 34a. Let $D \subset \mathbb{R}^{n}$ be a convex and open set, and let $f: D \rightarrow \mathbb{R}$ be a general convex with contact function. If for every $x^{*} \in \mathbb{R}^{n}$ inequality (23) holds, then we have $x^{*} \in g(\partial f(x))$.

8.4. Fundamental inequalities for general convexity. Since the general convex functions are defined by a functional inequality, it is not surprising that this notion will lead to a number of interesting and fundamental inequalities. Now we give some essential inequalities for general convex functions.

Theorem 35. Let $(X, \mathcal{M}, \mu)$ be a measure space such that $\mu(X)=1$, and let $J \subset \mathbb{R}$ be an open interval. Let $p: X \rightarrow J$ be an integrable function, and let $f: J \rightarrow \mathbb{R}$ be a general convex with contact function. If there is $x^{*} \in \mathbb{R}$ such that $g_{p(a, b)}\left(f_{y}^{\prime}(x)\right) \geq x^{*} y$ for all $y \in \mathbb{R}$, then

$$
\int_{X} f(p) d \mu \geq 2 f\left(\int_{X} p d \mu\right)-\max \{f(a), f(b), g(f(a), f(b))\}
$$

for every function $g: f(J)^{2} \rightarrow \mathbb{R}$ for which $f$ is a general convex with contact function and for two arbitrary points $a, b \in J$.

Proof. Put $t=\int_{X} p d \mu$. Clearly $t \in J$. Then from the preceding Theorem 34 a (inequality $\left(23^{\prime}\right)$ ) we obtain

$$
f(y) \geq 2 f(t)+x^{*}(y-t)-\max \{f(a), f(b), g(f(a), f(b))\}
$$

for all $y \in J$. Replacing $y$ by $p(x)$, and integrating over $x \in X$, we get thus the following inequality

$$
\int_{X} f(p) d \mu-2 f(t)+\max \{f(a), f(b), g(f(a), f(b))\} \geq x^{*}\left(\int_{X} p d \mu-t\right)=0,
$$


which is equivalent to the preceding inequality (GN). The proof is complete.

As an immediate consequence of Theorem 33 and of the preceding proof of Theorem 35, we are now in a position to formulate the following statement.

Theorem 36. Let $(X, \mathcal{M}, \mu)$ be a measure space such that $\mu(X)=1$, and let $J \subset \mathbb{R}$ be an open interval. Let $p: X \rightarrow J$ be an integrable function, and let $f: J \rightarrow \mathbb{R}$ be a general convex with tent function. Then the following inequality holds

$$
\begin{gathered}
\int_{X}\left|f(p)-f\left(\int_{X} p d \mu\right)\right| d \mu \geq \\
\geq f\left(\int_{X} p d \mu\right)-\max \left\{f(a), f\left(\int_{X} p d \mu\right), g\left(f(a), f\left(\int_{X} p d \mu\right)\right)\right\}
\end{gathered}
$$

for every function $g: f(J)^{2} \rightarrow \mathbb{R}$ for which $f$ is a general convex with tent function and for an arbitrary point $a \in J$.

Proof. Put $t=\int_{X} p d \mu$. Clearly $t \in J$. Take a $x^{*} \in g(\partial f(t))$. Then from inequality (GS) and by Theorem 33 for $b=t$ and $l=0$ we obtain

$$
|f(y)-f(t)| \geq f(t)+x^{*}(y-t)-\max \{f(a), f(t), g(f(a), f(t))\}
$$

for all $y \in J$. Replacing $y$ by $p(x)$, and integrating over $x \in X$, we get thus statement. The proof is complete.

Analogous to the preceding inequalities we obtain directly the following statements.

Theorem 37. Let $J:=[\alpha, \beta] \subset \mathbb{R}$ be an interval, let $f: J \rightarrow \mathbb{R}$ be a general convex with tent function and let $L$ be a set of all function $\psi: X \rightarrow \mathbb{R}(X$ is a nonempty set). If $A: L \rightarrow \mathbb{R}$ is a linear positive functional such that $A(1)=1$, then $f(\psi) \in L$ implies that $A(\psi) \in J$ for all $\psi \in L$, and

$A(|f(\psi)-f(A(\psi))|) \geq f(A(\psi))-\max \{f(a), f(A(\psi)), g(f(a), f(A(\psi)))\}$ for every function $g: f(J)^{2} \rightarrow \mathbb{R}$ for which $f$ is a general convex with tent function and for an arbitrary point $a \in J$.

Proof. Let $\alpha \leq \psi(t) \leq \beta$ for $t \in X$, then we obtain that $\alpha=A(\alpha)=$ $A(1 \cdot \alpha) \leq A(\psi(t)) \leq A(\beta)=\beta$. This means that $A(\psi) \in J$. Since $f$ is a general convex with tent function on $J$, thus we obtain that inequality (GS) holds for all $z, x \in(\alpha, \beta)$. If we take in (GS) $z=\psi(t), b=x, \lambda=0$ and $x=A(\psi)$, and if we apply the functional $A$, we obtain statement. Also, for points $a$ and $b$ similarly inequality holds. The proof is complete.

Theorem 38. Let $J \subset \mathbb{R}$ be an open interval, let $f: J \rightarrow \mathbb{R}$ be a general convex with contact function and let $L$ be a set of all function $\psi: X \rightarrow \mathbb{R}$ ( $X$ is a nonempty set). If $A: L \rightarrow \mathbb{R}$ is a linear positive functional such 
that $A(1)=1$, and if there is $x^{*} \in \mathbb{R}$ such that $g_{p(a, b)}\left(f_{y}^{\prime}(x)\right) \geq x^{*} y$ for all $y \in \mathbb{R}$, then

$\left(\mathrm{GN}^{\prime}\right)$

$$
A(f(\psi)) \geq 2 f(A(\psi))-\max \{f(a), f(b), g(f(a), f(b))\}
$$

for every function $g: f(J)^{2} \rightarrow \mathbb{R}$ for which $f$ is a general convex with contact function and for two arbitrary points $a, b \in J$.

Proof. Since $f$ is a general convex with contact function on $J$, from Theorem 34 , we obtain that inequality (23') holds. Therefore, if we take in (23') $z=\psi(t)$ and $x=A(\psi)$, and if we apply the functional $A$, we obtain $A(f(\psi)) \geq 2 f(A(\psi))+x^{*}(A(\psi)-A(\psi))-\max \{f(a), f(b), g(f(a), f(b))\}$

which is equivalent to the preceding inequality (GN'). The proof is complete.

On the other hand, as a directly consequence of the preceding statement, we obtain the following statement.

Theorem 39. Let $J:=(\alpha, \beta)$, let $\alpha<x_{1} \leq \cdots \leq x_{n}<\beta$ be real numbers and let $q_{1}, \ldots, q_{n}$ be real numbers such that for $k=1, \ldots, n$

$$
0 \leq \sum_{i=k}^{n} q_{i} \leq \sum_{i=1}^{n} q_{i}, \quad \sum_{i=1}^{n} q_{i}>0 .
$$

If $f: J \rightarrow \mathbb{R}$ is a general convex with contact function and if there is $x^{*} \in \mathbb{R}$ such that $g_{p(a, b)}\left(f_{y}^{\prime}(x)\right) \geq x^{*} y$ for all $y \in \mathbb{R}$, then

$$
(\mathrm{SN}) \frac{\sum_{i=1}^{n} q_{i} f\left(x_{i}\right)}{\sum_{i=1}^{n} q_{i}} \geq 2 f\left(\frac{\sum_{i=1}^{n} q_{i} x_{i}}{\sum_{i=1}^{n} q_{i}}\right)-\max \{f(a), f(b), g(f(a), f(b))\}
$$

for every function $g: f(J)^{2} \rightarrow \mathbb{R}$ for which $f$ is a general convex with contact function and for two arbitrary points $a, b \in J$.

This inequality is an evidently Jensen's type inequality. On the other hand, a corresponding inequality for integrals will also given as an integral analogue. First a very similarly inequality.

Theorem 40. Let $D \subset \mathbb{R}^{n}$ be a convex and open set, and let $f: D \rightarrow \mathbb{R}$ be a general convex with contact function. If there is $x^{*} \in \mathbb{R}^{n}$ such that $g_{p(a, b)}\left(f_{y}^{\prime}(x)\right) \geq x^{*} y$ for all $y \in \mathbb{R}$, then for every $x_{1}, \ldots, x_{n} \in D$ and for every nonnegative real numbers $q_{1}, \ldots, q_{n}$ with $\sum_{i=1}^{n} q_{i}>0$, we have (SN).

Theorem 41. Let $(X, \mathcal{M}, \mu)$ be a measure space and let $J \subset \mathbb{R}$ be an open interval. Let $p: X \rightarrow J$ and $q: X \rightarrow[0,+\infty]$ be integrable functions such that the product $p q$ is integrable and $\int_{X} q d \mu>0$. If $f: J \rightarrow \mathbb{R}$ is a general convex with contact function and if there is $x^{*} \in \mathbb{R}$ such that $g_{p(a, b)}\left(f_{y}^{\prime}(x)\right) \geq x^{*} y$ for all $y \in \mathbb{R}$, then

$$
\frac{\int_{X} q(f \circ p) d \mu}{\int_{X} q d \mu} \geq 2 f\left(\frac{\int_{X} p q d \mu}{\int_{X} q d \mu}\right)-\max \{f(a), f(b), g(f(a), f(b))\}
$$


for every function $g: f(J)^{2} \rightarrow \mathbb{R}$ for which $f$ is a general convex with contact function and for two arbitrary points $a, b \in J$.

8.5. Introduction and history of majorizations. G. H. Hardy, J. E. Littlewood and G. Pólya proved in 1929 the following majorization principle for convex functions which reads as follows.

Theorem H. Let $J \subset \mathbb{R}$ be an interval, let $x_{i}, y_{i} \in J(i=1, \ldots, n)$ be real numbers such that fulfilling

$$
\begin{gathered}
x_{1} \geq \cdots \geq x_{n}, \quad y_{1} \geq \cdots \geq y_{n} \\
\sum_{i=1}^{k} x_{i} \leq \sum_{i=1}^{k} y_{i} \quad(k=1, \ldots, n-1), \quad \sum_{i=1}^{n} x_{i}=\sum_{i=1}^{n} y_{i} .
\end{gathered}
$$

If $f: J \rightarrow \mathbb{R}$ is a convex function, then the following inequality holds in the adequate form as

$$
\sum_{i=1}^{n} f\left(x_{i}\right) \leq \sum_{i=1}^{n} f\left(y_{i}\right)
$$

Conversely, if for some $x_{i}, y_{i} \in J(i=1, \ldots, n)$ such that (1a) holds, and inequality $(H)$ is fulfilled for every convex function, then relations (2a) hold.

In $1932 \mathrm{~J} . \mathrm{K}$ a r a $\mathrm{m}$ a t a rediscovered Theorem H. Very similar to this statement was proved in 1949 by M. Tomić. We yl in 1949 in the same as M. To m i ć has obtained similar statement for $x_{i}>0$ and $y_{i}>0$, while M. Tomić did not require these restrictions. Practically, M. Tomić proved that a similar result contains in a certain sense Theorem H. He gave a geometrical proof for this, basing it on Gauss' theorem about the centroid.

For other generalizations of Theorem H of G. Pólya, L. F u c h s, T. P o p o v i c i u, K. $\mathrm{F}$ a $\mathrm{n}$ and some other see book of D. S. M i t r i n o v i ć: Analytic Inequalities (Sprin-gerVerlag, Berlin and New York, 1970.). In this section we consider the preceding problems for general convex functions.

With the help of the preceding facts in this part we present a new characterization of general convexity as a majorization principle. Second characterization of general convexity we give as an integral analogue of majorization principle without proof.

8.6. Inequalities alternative for general convexity. In this section we give some inequalities which are similar to well known inequality of HardyLittlewood-Pólya, i.e., similar with Theorem H.

We note that the preceding statements which are based upon it suggest the following question: what conditions must be satisfied by the two sets of numbers

$$
x_{1}, x_{2}, \ldots, x_{n} \text { and } y_{1}, y_{2}, \ldots, y_{n}
$$

in order that some inequality should be true for every function which is general convex with contact in an interval including all the numbers?

We are now in a position to formulate the following fundamental statement for general convex functions. 
Theorem 42. (Majorization principle). Let $J \subset \mathbb{R}$ be an interval, let $x_{i}, y_{i} \in J(i=1, \ldots, n)$ be real numbers such that fulfilling

$$
x_{1} \geq \cdots \geq x_{n}, \quad y_{1} \geq \cdots \geq y_{n},
$$

and let $p_{1}, \ldots, p_{n} \in \mathbb{R}$ be real numbers such that the following relations hold

$$
\sum_{i=1}^{k} p_{i} y_{i} \leq \sum_{i=1}^{k} p_{i} x_{i} \quad(k=1, \ldots, n-1), \quad \sum_{i=1}^{n} p_{i} x_{i}=\sum_{i=1}^{n} p_{i} y_{i} .
$$

If $f: J \rightarrow \mathbb{R}$ is a general convex with circled contact function for some function $g: f(J)^{2} \rightarrow \mathbb{R}$, then either

$$
\text { (A) } \sum_{i=1}^{n} p_{i} f\left(y_{i}\right) \leq 2 \sum_{i=1}^{n} p_{i} f\left(x_{i}\right)-\sum_{i=1}^{n} p_{i} \max \left\{f\left(x_{i}\right), f\left(y_{i}\right), g\left(f\left(x_{i}\right), f\left(y_{i}\right)\right)\right\}
$$

or

(B) $\sum_{i=1}^{n} p_{i} f\left(x_{i}\right) \geq 2 \sum_{i=1}^{n} p_{i} f\left(y_{i}\right)-\sum_{i=1}^{n} p_{i} \max \left\{f\left(x_{i}\right), f\left(y_{i}\right), g\left(f\left(x_{i}\right), f\left(y_{i}\right)\right)\right\}$.

Conversely, if $p_{i} \geq 0(i=1, \ldots, n)$, and if for some $x_{i}, y_{i} \in J(i=$ $1, \ldots, n)$ such that (24) holds and inequality (A) is fulfilled for every general convex with circled contact function $f: J \rightarrow \mathbb{R}$, then relations (25) hold.

Moreover, if $p_{i} \leq 0(i=1, \ldots, n)$ and if for some $x_{i}, y_{i} \in J(i=1, \ldots, n)$ such that (24) holds and inequality (B) is fulfiled for every general convex with circled contact function $f: J \rightarrow \mathbb{R}$, then relations (25) hold.

On the other hand, is connection with the preceding statement and Theorem 20 we are now in a position to formulate our main general statement.

Theorem 43. (Inequalities of alternative). Let $J \subset \mathbb{R}$ be an interval, let $x_{i}, y_{i} \in J(i=1, \ldots, n)$ be real numbers such that fulfilling

$$
x_{1} \geq \cdots \geq x_{n}, \quad y_{1} \geq \cdots \geq y_{n},
$$

and let $p_{1}, \ldots, p_{n} \in \mathbb{R}$ be real numbers such that the following relations hold

$$
\sum_{i=1}^{k} p_{i} y_{i} \leq \sum_{i=1}^{k} p_{i} x_{i} \quad(k=1, \ldots, n-1), \quad \sum_{i=1}^{n} p_{i} x_{i}=\sum_{i=1}^{n} p_{i} y_{i} .
$$

If $f: J \rightarrow \mathbb{R}$ is a general convex with contact function for some function $g: f(J)^{2} \rightarrow \mathbb{R}$, then either

$$
\sum_{i=1}^{n} p_{i} f\left(y_{i}\right) \leq 2 \sum_{i=1}^{n} p_{i} f\left(x_{i}\right)-\sum_{i=1}^{n} p_{i} \max \{f(a), f(b), g(f(a), f(b))\}
$$

or

$$
\sum_{i=1}^{n} p_{i} f\left(x_{i}\right) \geq 2 \sum_{i=1}^{n} p_{i} f\left(y_{i}\right)-\sum_{i=1}^{n} p_{i} \max \{f(a), f(b), g(f(a), f(b))\}
$$


for arbitrary $a, b \in J$. Conversely, if $p_{i} \geq 0(i=1, \ldots, n)$, and if for some $x_{i}, y_{i} \in J(i=1, \ldots, n)$ such that (26) holds and inequality $(N)$ or (M) is fulfilled for every general convex with contact function $f: J \rightarrow \mathbb{R}$, then relations (27) hold.

In further let the function $x \mapsto f(x)$ be nonnegative and integrable on $(0,1)$ so that it is measurable and finite almost everywhere and let $\mu(s)$ be the measure of the set on which $f(x) \geq s$. The function $x \mapsto f^{*}(x)$ which is inverse to $\mu$ is called the decreasing rearrangement of $f$. If $x, y \in L^{l}(0,1)$, we say that $y$ majorizes $x$, in writing $x \prec y$, if

$$
\begin{gathered}
\int_{0}^{s} x^{*}(t) d t \leq \int_{0}^{s} y^{*}(t) d t \text { for } 0<s<1, \\
\int_{0}^{1} x(t) d t=\int_{0}^{1} y(t) d t .
\end{gathered}
$$

We shall now give an integral inequality, which is connected with the majorization of functions, and which is analogue the preceding result, because without proof. We note G. H. Hardy, J. E. Littlewood and G. Pólya also proved an integral analogue of the inequality which appears in Theorem $\mathrm{H}$.

Theorem 43a. (Integral analogue of majorization). The following inequality of the form

$$
\int_{0}^{1} f(y(t)) d t \leq 2 \int_{0}^{1} f(x(t)) d t-\max \{f(a), f(b), g(f(a), f(b))\}
$$

or

$$
\int_{0}^{1} f(x(t)) d t \geq 2 \int_{0}^{1} f(y(t)) d t-\max \{f(a), f(b), g(f(a), f(b))\}
$$

holds for some function $g:[0,1]^{2} \rightarrow \mathbb{R}$, for arbitrary points $a, b \in[0,1]$, and for any general convex with contakt function $f$ if and only if $x$ majorizes $y$.

With this preceding statements we precision, correction and expand our the former majorization principles for general convex with contact functions.

8.7. Some further consequences. On the other hand, if to teasing on the convex class of functions taking

$$
\max \left\{f\left(x_{i}\right), f\left(y_{i}\right), g\left(f\left(x_{i}\right), f\left(y_{i}\right)\right)\right\}=\lambda f\left(x_{i}\right)+(1-\lambda) f\left(y_{i}\right)
$$

for arbitrary $\lambda \in[0,1]$ in Theorem 42, then from inequalities (A) and (B) we obtain the preceding Theorem $\mathrm{H}$ of Hardy-Littlewood-Pólya.

This means that Theorem 42 extends Theorem $\mathrm{H}$ to general convex functions. Also, from Theorem 42 as an immediate consequence we obtain the following statement for quasiconvex functions.

In this sense, a function $f: D \rightarrow \mathbb{R}$, where $D$ is a convex subset of $\mathbb{R}^{n}$, is said to be quasiconvex if

$$
f(\lambda x+(1-\lambda) y) \leq \max \{f(x), f(y)\}
$$


for all $x, y \in D$ and for arbitrary $\lambda \in[0,1]$. We notice that set of all quasiconvex functions can be a proper subset of the set all general convex with circled contact functions.

As an immediate application of the preceding Theorem 42 we obtain the following result.

Theorem 42a. (Majorization of quasiconvexity). Let $J \subset \mathbb{R}$ be an open interval, let $x_{i}, y_{i} \in J(i=1, \ldots, n)$ be real numbers such that fulfilling (26) and (27). If $f: J \rightarrow \mathbb{R}$ is an increasing quasiconvex function, then either

$$
\sum_{i=1}^{n} f\left(y_{i}\right) \leq 2 \sum_{i=1}^{n} f\left(x_{i}\right)-\sum_{i=1}^{n} \max \left\{f\left(x_{i}\right), f\left(y_{i}\right)\right\}
$$

or

$$
\sum_{i=1}^{n} f\left(x_{i}\right) \geq 2 \sum_{i=1}^{n} f\left(y_{i}\right)-\sum_{i=1}^{n} \max \left\{f\left(x_{i}\right), f\left(y_{i}\right)\right\} .
$$

Conversely, if for some $x_{i}, y_{i} \in J(i=1, \ldots, n)$ such that (26) holds and inequality ( $A$ ') is fulfilled for every quasiconvex function $f: J \rightarrow \mathbb{R}$, then relations (27) hold.

Proof. Taking $g\left(f\left(x_{i}\right), f\left(y_{i}\right)\right)=\max \left\{f\left(x_{i}\right), f\left(y_{i}\right)\right\}$ in inequalities (A) and (B) from Theorem 42 we obtain directly this statement for quasiconvex functions.

In connection with the preceding facts, since inequality $(\mathrm{N})$ or $(\mathrm{M})$ for $a \leq x_{i} \leq b(i=1, \ldots, n)$ and $p_{i}=1(i=1, \ldots, n)$ is equivalent only to inequality (M), thus we can Theorem 43 write in the following equivalent form in this case.

Theorem 43b. Let $J \subset \mathbb{R}$ be an open interval and let $x_{i}, y_{i} \in J(i=$ $1, \ldots, n)$ be real numbers such that fulfilling

$$
x_{1} \geq \cdots \geq x_{n}, \quad y_{1} \geq \cdots \geq y_{n} .
$$

Then, a necessary and sufficient condition in order that

$$
\sum_{i=1}^{n} f\left(x_{i}\right) \geq 2 \sum_{i=1}^{n} f\left(y_{i}\right)-n \max \{f(a), f(b), g(f(a), f(b))\}
$$

holds for every general convex function $f: J \rightarrow \mathbb{R}$ which is in contact with function $g: f(J)^{2} \rightarrow \mathbb{R}$ and for arbitrary $a, b \in J\left(a \leq x_{i} \leq b\right.$ for $i=1, \ldots, n)$, is that

$$
\sum_{i=1}^{k} y_{i} \leq \sum_{i=1}^{k} x_{i} \quad(k=1, \ldots, n-1), \quad \sum_{i=1}^{n} y_{i}=\sum_{i=1}^{n} x_{i} .
$$


As an immediate consequence of Theorem 42, directly, we obtain the following inequality. Indeed, putting in (B) $y_{1}=\cdots=y_{n}=n^{-1} \sum_{i=1}^{n} x_{i}$ we get

$$
\sum_{i=1}^{n} f\left(x_{i}\right) \geq
$$

$\geq 2 n f\left(\frac{1}{n} \sum_{i=1}^{n} x_{i}\right)-\sum_{i=1}^{n} \max \left\{f\left(x_{i}\right), f\left(\frac{1}{n} \sum_{i=1}^{n} x_{i}\right), g\left(f\left(x_{i}\right), f\left(\frac{1}{n} \sum_{i=1}^{n} x_{i}\right)\right)\right\}$

for every general convex function $f: J \rightarrow \mathbb{R}(J \subset \mathbb{R}$ is an open interval) which is in circled contact with function $g: f(J)^{2} \rightarrow \mathbb{R}$.

This inequality is a generalization of Jensen's inequality for convex functions. Indeed, if to teasing on the convex class of functions taking $(\mathrm{C})$, then from (Gc) we get Jensen's inequality.

The following statement is very similar to Theorem 42 .

Theorem 42b. Let $J \subset \mathbb{R}$ be an open interval, let $x_{i}, y_{i} \in J(i=1, \ldots, n)$ be real numbers such that fulfilling (28) and such that

$$
\sum_{i=1}^{k} y_{i} \leq \sum_{i=1}^{k} x_{i} \quad(k=1, \ldots, n) .
$$

If $f: J \rightarrow \mathbb{R}$ is an increasing general convex with circled contact function for some function $g: f(J)^{2} \rightarrow \mathbb{R}$ then either

$$
\sum_{i=1}^{n} f\left(y_{i}\right) \leq 2 \sum_{i=1}^{n} f\left(x_{i}\right)-\sum_{i=1}^{n} \max \left\{f\left(x_{i}\right), f\left(y_{i}\right), g\left(f\left(x_{i}\right), f\left(y_{i}\right)\right)\right\} .
$$

or

$$
\sum_{i=1}^{n} f\left(x_{i}\right) \geq 2 \sum_{i=1}^{n} f\left(y_{i}\right)-\sum_{i=1}^{n} \max \left\{f\left(x_{i}\right), f\left(y_{i}\right), g\left(f\left(x_{i}\right), f\left(y_{i}\right)\right)\right\} .
$$

Conversely, if for some $x_{i}, y_{i} \in J(i=1, \ldots, n)$ such that (28) holds and inequality $(\mathrm{Aa})$ is fulfilled for every increasing general convex with circled contact function $f: J \rightarrow \mathbb{R}$, then inequalities (I) hold.

This proof of this statement is totally analogous to the preceding proof of Theorem 42. The following statement is very similar to Theorem 43b.

Theorem 43c. Let $J \subset \mathbb{R}$ be an open interval and let $x_{i}, y_{i} \in J(i=$ $1, \ldots, n)$ be real numbers such that fulfilling

$$
x_{1} \geq \cdots \geq x_{n}, \quad y_{1} \geq \cdots \geq y_{n} .
$$

Then, a necessary and sufficient condition in order that

$$
\sum_{i=1}^{n} f\left(x_{i}\right) \geq 2 \sum_{i=1}^{n} f\left(y_{i}\right)-n \max \{f(a), f(b), g(f(a), f(b))\}
$$


holds for every increasing general convex function $f: J \rightarrow \mathbb{R}$ which is in contact with function $g: f(J)^{2} \rightarrow \mathbb{R}$ and for arbitrary $a, b \in J\left(a \leq x_{i} \leq b\right.$ for $i=1, \ldots, n)$, is that

$$
\sum_{i=1}^{k} y_{i} \leq \sum_{i=1}^{k} x_{i} \quad(k=1, \ldots, n) .
$$

The proof of this statement is very similar and totally analogous to the preceding proof of Theorem 43.

8. Further applications. In this part we give some consequences of the preceding results of majorizations in the following forms:

1. (Extension of Lim's inequality). Let $x>0, y>0, z>x+y$ be real numbers, and let $f: \mathbb{R}_{+}^{0} \rightarrow \mathbb{R}$ be a general convex with contact function. Then

$$
f(x)+f(y+z) \geq 2[f(x+y)+f(z)]-2 \max \{f(a), f(b), g(f(a), f(b))\}
$$

for every function $g: f\left(\mathbb{R}_{+}^{0}\right)^{2} \rightarrow \mathbb{R}$ which is in contact with $f$ and for arbitrary points $a, b \in \mathbb{R}_{+}^{0}(a \leq x, y+z \leq b)$.

2. (Extension of Petrović's inequality). Let $J \subset \mathbb{R}$ be an open interval, and let $f: J \rightarrow \mathbb{R}$ be a general convex with contact function. If $x_{1}, \ldots, x_{n} \in J$, then the following inequality holds

$$
\begin{gathered}
f\left(x_{1}+\cdots+x_{n}\right)+(n-1) f(0) \geq \\
\geq 2\left[f\left(x_{1}\right)+\cdots+f\left(x_{n}\right)\right]-n \max \{f(a), f(b), g(f(a), f(b))\}
\end{gathered}
$$

for every $n \in \mathbb{N}$, for every function $g: f(J)^{2} \rightarrow \mathbb{R}$ which is in contact with $f$ and for arbitrary $a, b \in J\left(a \leq x_{1}+\cdots+x_{n}, 0 \leq b\right)$.

3. (An inequality of general convexity). Let $J \subset \mathbb{R}$ be an open interval, and let $f: J \rightarrow \mathbb{R}$ be a general convex with contact function. Then

$$
\begin{gathered}
f(x)+f(y)+f(z)+3 f\left(\frac{x+y+z}{3}\right) \geq \\
\geq 4\left[f\left(\frac{x+y}{2}\right)+f\left(\frac{y+z}{2}\right)+f\left(\frac{x+z}{2}\right)\right]-6 \max \{f(a), f(b), g(f(a), f(b))\}
\end{gathered}
$$

for every function $g: f(J)^{2} \rightarrow \mathbb{R}$ which is in contact with $f$ and for arbitrary points $a, b \in J(a \leq x, y, z \leq b)$.

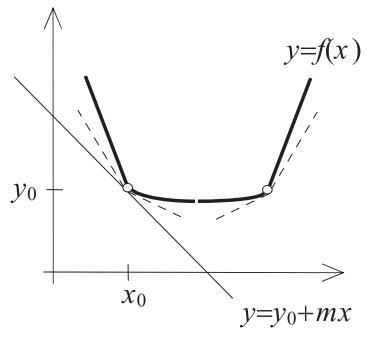

FIGURE 7

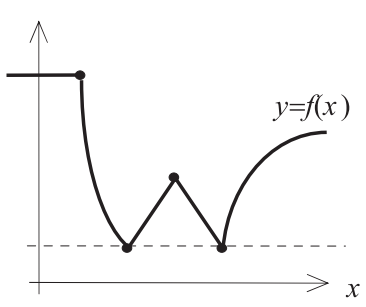

FIGURE 8

4. (Hermite-Hadamard type inequalities). Let $J \subset \mathbb{R}$ be an open interval, let $f$ : $J \rightarrow \mathbb{R}$ be a general convex with contact function, and let there exists $x^{*} \in \mathbb{R}$ such that 
$g_{p(a, b)}\left(f_{y}^{\prime}(x)\right) \geq x^{*} y$ for every $y \in \mathbb{R}$. If $a<b(a, b \in J)$ then

$$
\begin{aligned}
& 2 f\left(\frac{a+b}{2}\right)-\max \{f(\lambda), f(\rho), g(f(\lambda), f(\rho))\} \leq \frac{1}{b-a} \int_{a}^{b} f(x) d x \leq \\
& \leq \max \{f(a), f(b), g(f(a), f(b))\}
\end{aligned}
$$

for every function $g: f(J)^{2} \rightarrow \mathbb{R}$ under which $f$ is a general convex with contact function, and for two arbitrary points $\lambda, \rho \in J$. Also, then

$$
2 f\left(\frac{a+b}{2}\right)-\max \{f(\lambda), f(\rho), g(f(\lambda), f(\rho))\} \leq \frac{1}{(b-a)^{2}} \int_{a}^{b} \int_{a}^{b} f\left(\frac{x+y}{2}\right) d x d y
$$

On the other hand, if $f: J \rightarrow \mathbb{R}(J \subset \mathbb{R}$ is an open interval) is a convex functional and if $a<b(a, b \in J)$, then by $\mathrm{D} \mathrm{r}$ a g o $\mathrm{m}$ i $\mathrm{r}$ [1992] we have

$$
f\left(\frac{a+b}{2}\right) \leq \frac{1}{(b-a)^{2}} \int_{a}^{b} \int_{a}^{b} f\left(\frac{x+y}{2}\right) d x d y \leq \frac{1}{b-a} \int_{a}^{b} f(x) d x .
$$

5. (Connection of convexity and general convexity). We notice that the set of all convex and quasiconvex functions can be a proper subset of the set of all general convex functions. See Figs. 7, 8, 9, 10.

Let $G K(J)$ be class of all general convex functions with contact and let $K(J)$ be class of all convex functions on an open interval $J \subset \mathbb{R}$.

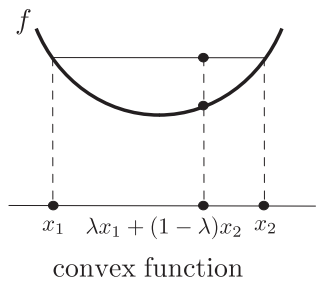

Figure 9

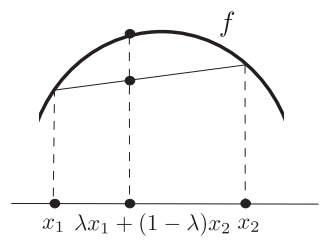

concave function

Theorem 44 (Relation of $G K(J)$ and $K(J))$ ). Let $J \subset \mathbb{R}$ be an open interval, let $x_{i}, y_{i} \in J(i=1, \ldots, n)$ be are real numbers such that (26), and let $p_{1}, \ldots, p_{n} \in \mathbb{R}$ be nonnegative numbers such that (27). Then for every convex function $f: J \rightarrow \mathbb{R}$ the following inequality holds

$$
\sum_{i=1}^{n} p_{i} f\left(y_{i}\right) \leq \sum_{i=1}^{n} p_{i} f\left(x_{i}\right)
$$

if and only if for every general convex function with contact $f: J \rightarrow \mathbb{R}$ the following inequality holds in the form as

$$
\sum_{i=1}^{n} p_{i} f\left(x_{i}\right) \geq 2 \sum_{i=1}^{n} p_{i} f\left(y_{i}\right)-\left(\sum_{i=1}^{n} p_{i}\right) \max \{f(a), f(b), g(f(a), f(b))\}
$$

for every function $g: f(J)^{2} \rightarrow \mathbb{R}$ under which $f$ is a general convex function with contact and for arbitrary two points $a, b \in J\left(a \leq x_{i} \leq b\right.$ for $\left.i=1, \ldots, n\right)$.

The proof of this statement directly it follows from Theorems $\mathrm{H}$ and 43 . For further facts see: Taskovi ć [2001]. 


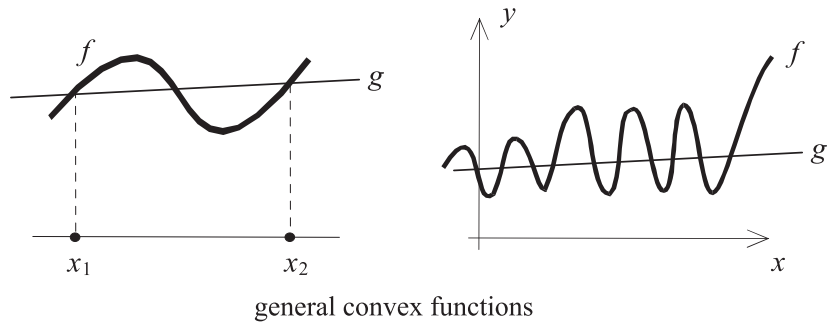

FiguRE 10

We notice, if $f: J \rightarrow \mathbb{R}(J \subset \mathbb{R}$ is an open interval) is a convex function and if $a<b$ $(a, b \in J)$, then the following inequalities hold in the form as

$$
f\left(\frac{a+b}{2}\right) \leq \frac{1}{b-a} \int_{a}^{b} f(x) d x \leq \frac{f(a)+f(b)}{2},
$$

well-known as Hermite-Hadamard inequalities (or only as Hadamard inequality). For history of inequalities (H) see: M i tr i n o v i ć-P e č a r i ć-F i n k [1993], P i c a r d [1905], J or d a n-M an sion [1901], B e cken b a ch [1948], Fe jér [1906], and Tas k ovi ć [2001].

6. (Inequalities for means). Let $F$ be a strictly monotonic function on an interval $J \subset \mathbb{R}$. let $p_{1}, \ldots, p_{n}$ be nonnegative real numbers such that $p_{1}+\cdots+p_{n}>0$, and let $a_{1}, \ldots, a_{n} \in F(J)$ be arbitrary points. The expression

$$
M_{n}(F ; a, p)=F\left(\frac{\sum_{i=1}^{n} p_{i} F^{-1}\left(a_{i}\right)}{\sum_{i=1}^{n} p_{i}}\right) .
$$

is a called a quasiarithmetic weighted mean of $a_{1}, \ldots, a_{n}$ with the weights $p_{1}, \ldots, p_{n}$. In $(25)$ and in the sequel $a$ stands for $\left(a_{1}, \ldots, a_{n}\right)$ and $p$ stands for $\left(p_{1}, \ldots, p_{n}\right)$.

Theorem 45. Let $J \subset \mathbb{R}$ be an open interval, and let the functions $F, G$ : $J \rightarrow \mathbb{R}$ be continuous strictly monotonic $F(J)=G(J)$, and let the function $G^{-1}(F)$ be general J-convex. If $G$ is a decreasing function, then there is a function $d: J^{n} \rightarrow J$ such that

$$
M_{n}(F ; a, p) \geq \min \left\{a_{1}, \ldots, a_{n}, G\left(d\left(G^{-1}\left(a_{1}\right), \ldots, G^{-1}\left(a_{n}\right)\right)\right)\right\}
$$

for every $n \in \mathbb{N}$, for all $a_{1}, \ldots, a_{n} \in F(J)$, and for all nonnegative numbers $p_{1}, \ldots, p_{n}$ such that $p_{1}+\cdots+p_{n}>0$. If $G$ is an increasing function, then there is a function $g: J^{n} \rightarrow J$ such that

$$
M_{n}(F ; a, p) \leq \max \left\{a_{1}, \ldots, a_{n}, G\left(g\left(G^{-1}\left(a_{1}\right), \ldots, G^{-1}\left(a_{n}\right)\right)\right)\right\}
$$

for every $n \in \mathbb{N}$, for all points $a_{1}, \ldots, a_{n} \in F(J)$, and for arbitrary numbers $p_{1}, \ldots, p_{n} \in \mathbb{R}_{+}^{0}$ such that $p_{1}+\cdots+p_{n}>0$.

We will give one more generalization of the inequality between means. Let $F$ be a strictly monotonic function on an interval $D \subset \mathbb{R}$, let $n \in \mathbb{N}$, 
let $I$ be a subset of $\{1, \ldots, n\}$, let $a_{1}, \ldots, a_{n} \in F(D)$, and let $p_{1}, \ldots, p_{n} \geq 0$ such that $\sum_{i \in I} p_{i}>0$. If $H: F(D) \rightarrow \mathbb{R}$ is a continuous function, put

$$
\alpha(H ; F ; a, p, I)=H \circ F\left(\frac{\sum_{i \in I} p_{i} F^{-1}\left(a_{i}\right)}{\sum_{i \in I} p_{i}}\right) .
$$

With this notation, and under the conditions specified, we have the following statement.

Theorem 46. (General convexity of index set). If the function $F$ is continuous, the function $H(F)$ is general J-convex, and if $I, J$ are disjoint subset of $\{1, \ldots, n\}$, then there is a function $g: H(F)^{2} \rightarrow \mathbb{R}$ such that

$$
\alpha(F, I \cup J) \leq \max \{\alpha(F, I), \alpha(F, J), g(\alpha(F, I), \alpha(F, J))\}
$$

where $\alpha(F, I)=\alpha) H, F ; a, p, I)$ for fixed $a$ and $p$. If $H(F)$ is a general $J$-concave function, then there is a function $d: H(F)^{2} \rightarrow \mathbb{R}$ such that

$$
\alpha(F, I \cup J) \geq \min \{\alpha(F, I), \alpha(F, J), d(\alpha(F, I), \alpha(F, J))\}
$$

7. (A new Method for Inequalities, Tasković [2001]). Today inequalities play a significiant role in all fields of mathematics, and they present a very active and attractive field of research.

In this part we give a method for proving general convex type inequalities. We consider our general method (via a fixed point) on the following inequality.

Hölder-Young inequality. Let $x, y \in \mathbb{R}_{+}^{0}$ and $1<p<+\infty$ such that the following equality holds $1 / p+1 / q=1$. Then the following inequality holds in the form as

$$
x^{1 / p} y^{1 / q} \leq \frac{x}{p}+\frac{y}{q},
$$

with equality holding if and only if $x=y$.

Method of Proof. In Theorem 2.2 (or Lemma 2.1a) we may choose for the function $g: P \rightarrow P\left(P:=\mathbb{R}_{+}\right)$that is $g(t)=\left(x^{1 / p} y^{1 / q}\right)^{2} / t$, where $\xi=x^{1 / p} y^{1 / q}$ is a fixed point of $g$. Then, from Lemma $2.1 \mathrm{a}$, we obtain

$$
\xi=x^{1 / p} y^{1 / q} \leq \max \{\lambda, g(\lambda)\}
$$

for arbitrary $\lambda \in \mathbb{R}_{+}$. Thus, for $\lambda=x / p+y / q$ we have two cases. First, if $\max \{\lambda, g(\lambda)\}=$ $x / p+y / q$, then inequality (37) holds, If not, then from (38) we obtain

$$
\xi=x^{1 / p} y^{1 / q} \leq g\left(\frac{x}{p}+\frac{y}{q}\right)=\frac{\left(x^{1 / p} y^{1 / q}\right)^{2}}{\frac{x}{p}+\frac{y}{q}} .
$$

But, this is not possible by Theorem 2.2, since $g$ is a decreasing function, which tends to zero. Thus, the inequality (37) holds.

Annotations. The method of the preceding proof of Hölder-Young inequality can be used for all general convex type inequalities by: Hadamard, Jensen, Abel, Hölder, Cauchy, Young, Karamata, Grüss, and many others. See: M i t r i n o vi ć [1970], M i t r i n o vi ć et al. [1993] and Tas k ovi ć [2001].

Also, the preceding method is to be based on the following result. 
Theorem 47. (Equality for Functionals, Task ovi ć [1986b]). Let $S$ be a nonempty set and let $f, g: S \rightarrow S$ be two mappings. If $A: f(S) \times g(S) \rightarrow \mathbb{R}_{+}$, then the following fact holds in the form as

$$
A(f, g)=\max _{\lambda \in \mathbb{R}_{+}} \min \left\{\lambda, \frac{(A(f, g))^{2}}{\lambda}\right\}=\min _{\lambda \in \mathbb{R}_{+}} \max \left\{\lambda, \frac{(A(f, g))^{2}}{\lambda}\right\} .
$$

The proof of this equality may be found in Tasković [1986b] and Mitri n ović et al. [1993]. Also see: Ta s kovi ć [2001].

We notice that the following consequence of $(G)$ has many applications in the theory of inequalities in the forma as:

$$
\min \left\{\lambda, \frac{(A(f, g))^{2}}{\lambda}\right\} \leq A(f, g) \leq \max \left\{\lambda, \frac{(A(f, g))^{2}}{\lambda}\right\}
$$

for arbitrary $\lambda \in \mathbb{R}_{+}$. Main applications of inequality (G') are for obtained Grüss's and Karamata's inequalities. See: Mi tri nović et al. [1993] and Tas k ovi ć [2001]. ${ }^{3}$

In connection with the preceding facts, from Theorem 47 , we have the following statement as a new double inequality in the form as:

Theorem 48. (Tasković, [2001]). Let $X$ be a real linear space of functions defined on an interval $J \subset \mathbb{R}$, and let the functions $f, g, f g \in X$ satisfying $0<m \leq f(x) \leq M$ and $0<r \leq g(x) \leq R$ for every $x \in J$. If $A: X \rightarrow \mathbb{R}$ is a linear functional such that $A(1)-1$, then

$$
\frac{1}{\lambda(m, r, M, R)} \leq \frac{A(f g)}{A(f) A(g)} \leq \lambda(m, r, M, R)
$$

for every $\lambda \geq \xi(f, g)$, where $\xi(f, g)$ is a fixed point of the function in the following adequate form as $\psi(t)=t^{-1}\left[A(f g)^{2}\right](A f A g)^{-1}: \mathbb{R}_{+} \rightarrow \mathbb{R}_{+}$.

We notice in the special case of the preceding inequalities we obtain a result of $\mathrm{L} \mathrm{u} \mathrm{p} \mathrm{a} \mathrm{s}$ [1978] for $\lambda=K^{2}$, where

$$
K=\frac{\sqrt{m r}+\sqrt{M R}}{\sqrt{m R}+\sqrt{r M}} .
$$

On the other hand, the inequalities (39) are a directly extension of the following statement in the form as:

Theorem 49. (Karamata, [1948]). Let $f$ and $g$ be are integrable functions on $(0,1)$ such that $0<m \leq f(x) \leq M$ and $0<r \leq g(x) \leq R$ for every $0 \leq x \leq 1$. Then

$$
\frac{1}{K^{2}} \leq \frac{\int_{0}^{1} f(x) g(x) d x}{\int_{0}^{1} f(x) d x \int_{0}^{1} g(x) d x} \leq K^{2}
$$

for the constant $K$ defined in (40). In this case, the constant $K$ is the best possible.

Further, we can to write down, that from Theorem 47 we obtain the following general inequalities as an extension of the former results.

Theorem 50. (Global Inequalities, Tasković [1994]). Let X be a real linear space of real functions defined on an interval $J \subset \mathbb{R}$, and let the functions $f, g \in X$ and the functional $F: X \rightarrow \mathbb{R}$ satisfying $m \leq f(x) \leq M(m \neq M)$ and $0<r \leq F(g) \leq R$. If $B: X \times X \rightarrow \mathbb{R}$, then

$$
\frac{m r D(f)+M r d(f)}{R D(f)+r d(f)} \leq B(f, g) \leq \frac{M R d(f)+m r D(f)}{R d(f)+r D(f)} .
$$

${ }^{3}$ Historical facts. The inequality (37) was first proved by O t t o-H öld e r (18591937) in 1889 for series. The extension to integrals is due to Fr y g e s R i es z. For other facts see: Young [1912] and M itrinović et al. [1993]. 
where the following conditions hold: $D(f):=M-F(f) \geq d(f):=F(f)-m$ and $R^{2} d(f) \geq$ $r^{2} D(f)$ or $D(f) \leq d(f)$ and $R^{2} D(f) \geq r^{2} d(f)$.

In the special case of inequalities ( $\check{S})$ we obtain well-known so-called Karamata's inequalities in the following form.

Theorem 51. (Karamata, [1933]). Let $f(x)$ and $g(x)$ be are integrable functions on $(0,1)$ and let $m \leq f(x) \leq M$ for every $x \in[0,1]$, then the following inequalities hold in the form as

$$
\frac{\lambda m(M-\mu)+M(\mu-m)}{\lambda(M-\mu)+(\mu-m)} \leq \frac{\int_{0}^{1} f(x) g(x) d x}{\int_{0}^{1} g(x) d x} \leq \frac{m(M-\mu)+\lambda M(\mu-m)}{(M-\mu)+\lambda(\mu-m)}
$$

for every function $0<a \leq g(x) \leq \lambda a(\lambda>1)$ with the $x \in[0,1]$, where $\mu$ is the integral of the function $f(x)$ on $[0,1]$.

We notice that an extension of inequalities (K) is given by L u p a s [1978] for linear positive functionals $F: X \rightarrow \mathbb{R}$ with $F(1)=1$. Also, in connection with this, see: $\mathrm{F}$ in k [1977], P ó l y a-S z e g ö [1925], and P e č a r i ć-S a vi ć [1984].

Further, as a special case of the Global Inequalities $(\check{\mathrm{S}})$, we have directly the following statement via constant $K$ in (40), in the form as:

Corollary 8. (Tasković, [2001, p. 597]). Let $X$ be a real linear space of real functions defined on interval $J \subset \mathbb{R}$, let $F: X \rightarrow \mathbb{R}$ satisfying $0<m \leq F(f) \leq M$ and $0<r \leq$ $F(g) \leq R$ for $f, g \in X$. Then for the constant $K$ in (40) and $B: X \times X \rightarrow \mathbb{R}_{+}$, then the following inequalities hold

$$
\frac{1}{K^{2}} \leq \frac{B(f, g)}{F(f)} \leq K^{2}
$$

where the following conditions hold: $M-F(f) \geq F(f)-m$ and $R^{2}(F(x)-m) \geq r^{2}(M-$ $F(f))$ or $M-F(f) \leq F(f)-m$ and $R^{2}(M-F(f)) \geq r^{2}(F(f)-m)$.

8. (Grüss' Inequality). Further let $\varphi \leq f(x) \leq \phi, \gamma \leq g(x) \leq \Gamma$ for every $x \in[a, b]$, where $\varphi, \phi, \gamma$ and $\Gamma$ are given real constants. In 1935 G. G r ü s s stated the hypothesis that

$$
\left|\frac{1}{b-a} \int_{a}^{b} f(x) g(x) d x-\frac{1}{(b-a)^{2}} \int_{a}^{b} f(x) d x \int_{a}^{b} g(x) d x\right| \leq \frac{1}{4}(\phi-\varphi)(\Gamma-\gamma) .
$$

We notice, also in 1935 G. G. G r ü s s proved that (Gs) is valid and that the constant $1 / 4$ is the best possible. For furhter facts see: L a n d a u [1935], H a r d y [1936], K a r a m a t a [1948], K n o p p [1935], Fe m p l [1965], and M i t r i n ovi ć-P eč a r i ć-F in k [1993].

In connection with the preceding facts, applying Theorem 50 on the functional $B(f, g)$ we directly obtain the following inequality in the form as:

$$
B(f, g)=\left|\int_{0}^{1} f(x) g(x) d x-\int_{0}^{1} f(x) d x \int_{0}^{1} g(x) d x\right| \leq \frac{R M d(f)+r m D(f)}{R d(f)+r D(f)} .
$$

\section{Representation of General CONVEXity}

This section is continue the study and considerations of the general convex functions, which are introduced in the former paper by Task ovi ć: Math. Japonica, 37 (1992), 367-372. 
The following main result is proved: Every continuous general convex with contact (for some continuous function $\left.g: f(I)^{2} \rightarrow \mathbb{R}, I:=[a, b]\right)$ function $f:[a, b] \rightarrow \mathbb{R}$ is the uniform limit of the sequence

$$
\psi_{n}(x)=\sum_{k=0}^{n} p_{k} \omega\left(x, x_{k}\right) \quad \text { for } \quad n=1,2, \ldots
$$

where $\omega\left(x, x_{k}\right)=\left(x-x_{k}\right)_{+}$or $\omega\left(x, x_{k}\right)=\left|x-x_{k}\right|$, where $x_{k} \in[a, b]$ for $k=0,1, \ldots, n$ and where the $p_{i} \in \mathbb{R}(i=0,1, \ldots, n)$ satisfies the following condition

$$
2 p_{j}-\left(\sum_{k=0}^{j-1} p_{k}-\sum_{k=j}^{n} p_{k}\right) \geq 0 \quad(j=1, \ldots, n-1) .
$$

Here $x_{+}=0$ if $x<0$ and $x_{+}=x$ if $x>0$. Also, every function of the sequence (S) is a general convex with contact function.

9.1. Approximation of general convex functions. In this section we continue the study and considerations of the general convex functions. In this sense we note that a number of well known classical statements in 1916 of Galvani, Hyers-Ulam in 1952, Toda in 1936 and Popoviciu in 1965 consider the problem of approximation and representation of convex functions by picewise affine functions or as some stability statement.

In this part the problem of approximation and representation of general convex functions is studied and considered.

We notice that a large number of statements from the theory of general convex functions are of the following form: Af is a general convex function, where $f$ is a general convex function and $A$ a given linear operator. In this paper we consider this problem and his applications too.

In this section the problem of approximation and representation of general convex functions is considered. In this sense we are now in a position to formulate main statement as a solution of the preceding problem of representation. First we note that the following fact holds.

Lemma 5. Let $D \subset \mathbb{R}^{n}$ be a convex and open set, and let $f_{n}: D \rightarrow \mathbb{R}$ (for $n \in \mathbb{N}$ ) be a sequence of general convex functions for some continuous function $g: f_{n}(D)^{2} \rightarrow \mathbb{R}$. If the sequence $\left\{f_{n}\right\}_{n \in \mathbb{N}}$ converges in $D$ to a finite function $f$, then $f$ is a general convex function for the continuous function $g: f(D)^{2} \rightarrow \mathbb{R}$.

In connection with the preceding Lemma 5, we notice, moreover, the convergence is uniform on any closed subinterval I of $D$.

In this section we shall introduce some notations. In this sense, let $-\infty<$ $a<b<+\infty$ and let $I:=[a, b]$. We shall considered general convex functions which are, de facto, continuous on open interval $(a, b)$.

Theorem 52. (Representation of general convexity). Every continuous gene-ral convex with contact (for some continuous function $g: f(I)^{2} \rightarrow \mathbb{R}$ ) 
function $f:[a, b] \rightarrow \mathbb{R}$ is the uniform limit of the sequence

$$
\psi_{n}(x)=\sum_{k=0}^{n} p_{k} \omega\left(x, x_{k}\right) \quad \text { for } \quad n=1,2, \ldots
$$

where $\omega\left(x, x_{k}\right)=\left(x-x_{k}\right)_{+}$or $\omega\left(x, x_{k}\right)=\left|x-x_{k}\right|$, where $x_{k} \in[a, b]$ for $k=0, \ldots, n$ and where the $p_{i} \in \mathbb{R}(i=0, \ldots, n)$ satisfies the following condition

$$
2 p_{j}-\left(\sum_{i=0}^{j-1} p_{i}-\sum_{i=j}^{n} p_{i}\right) \geq 0 \quad(j=1, \ldots, n-1) .
$$

Here $x_{+}=0$ if $x<0$ and $x_{+}=x$ if $x>0$. Also, every function of the sequence $(S)$ is a general convex with contact function.

We notice, if $f:[a, b] \rightarrow \mathbb{R}$ is a convex function, then a necessary and sufficient condition for $\left\{\psi_{n}\right\}_{n \in \mathbb{N}}$ to be convex is that $p_{j}>0(j=0, \ldots, n)$. Precisely, representation of a convex function on $[a, b]$ is the uniform limit of the sequence $(\mathrm{S})$ where $p_{i}>0(i=0, \ldots, n)$. This statement was proved in 1936 by Toda and in 1965 by Popoviciu. First the coeficients $p_{k}$ ( $k=$ $0, \ldots, n)$ are explicitly given, while in 1965 the uniform convergence of the sequence $\left\{\psi_{n}\right\}_{n \in \mathbb{N}}$ was proved.

Proof of Theorem 52 Let the interval $[a, b]$ be divided by equidistant $\sigma_{n}(I): a=x_{0}<x_{1}<\cdots<x_{n}=b$ division, i.e., let

$$
x_{k}=a+k h, \quad h=\frac{b-a}{n} \text { for } k=0,1, \ldots, n .
$$

Then, if the sequence of functions $\left\{\psi_{n}\right\}_{n \in \mathbb{N}}$ defined by the following equality

$$
\psi_{n}(x)=\sum_{k=0}^{n} p_{k}\left|x-x_{k}\right|,
$$

interpolate the function $f$ in points $x_{k}$ i.e., if $\psi_{n}\left(x_{k}\right)=f\left(x_{k}\right)$ for $k=$ $0,1, \ldots, n$ we obtain the following equalities

$$
\psi_{n}\left(x_{j}\right)=h \sum_{k=0}^{n}|j-k| p_{k} \quad(0 \leq j \leq n) .
$$

Let $t_{j}(i=1, \ldots, n-1)$ denoted the following sum in form

$$
2 p_{j}-\left(\sum_{i=0}^{j-1} p_{i}-\sum_{i=j}^{n} p_{i}\right) \quad \text { for } \quad j=1, \ldots, n-1 .
$$

Thus, since $f$ is a general convex with contact function from Theorem 19 we obtain the following sequences of equalities and inequalities for $x_{j-1}<$ 


$$
\begin{gathered}
x_{j}<x_{j+1}(j=1, \ldots, n-1): \\
0 \leq \frac{f\left(x_{j+1}\right)-2 f\left(x_{j}\right)+\max \left\{f\left(x_{j}-1\right), f\left(x_{j+1}\right), g\left(f\left(x_{j-1}\right), f\left(x_{j+1}\right)\right)\right\}}{x_{j+1}-x_{j}}- \\
-\frac{f\left(x_{j}\right)-2 f\left(x_{j-1}\right)+\max \left\{f\left(x_{j-1}\right), f\left(x_{j+1}\right), g\left(f\left(x_{j-1}\right), f\left(x_{j+1}\right)\right)\right\}}{x_{j}-x_{j-1}}=\cdots \\
\ldots=2 p_{j}-\left(\sum_{k=0}^{j-1} p_{k}-\sum_{k=j}^{n} p_{k}\right):=t_{j} \quad \text { for } \quad j=1, \ldots, n-1 .
\end{gathered}
$$

This means that $t_{j} \geq 0(j=1, \ldots, n-1)$. If $x \in[a, b]$, then there is a segment $\left[x_{k}, x_{k+1}\right](k=0, \ldots, n-1)$ such that $x \in\left[x_{k}, x_{k+1}\right]$. If $\omega_{f}(\delta)$ denoted the modulus of continuity of the function $f$ on segment with distance $d$, then since $\psi_{n}(x)$ to coincide with the affine function in points $\left(x_{k}, f\left(x_{k}\right)\right)$ and $\left(x_{k+1}, f\left(x_{k+1}\right)\right)$, we obtain the following equalities and inequalities:

$$
\begin{aligned}
& \left|f(x)-\psi_{n}(x)\right|=\left|f(x)-f\left(x_{k}\right)-\frac{x-x_{k}}{x_{k+1}-x_{k}}\left(f\left(x_{k+1}\right)-f\left(x_{k}\right)\right)\right| \leq \\
& \leq\left|f(x)-f\left(x_{k}\right)\right|+\frac{x-x_{k}}{x_{k+1}-x_{k}}\left|f\left(x_{k+1}\right)-f\left(x_{k}\right)\right| \leq 2 \omega_{f}\left(\frac{b-a}{n}\right) .
\end{aligned}
$$

Since $\omega_{f}\left(\frac{b-a}{n}\right)$ converges to zero when $n \rightarrow \infty$ we have that $\left\{\psi_{n}(x)\right\}_{n \in \mathbb{N}}$ converges uniformly toward the function $f$, which on the basis Lemma 5 , is a general convex function. The proof is complete.

In connection with the preceding proof of Theorem 52, we notice, that directly the following statement holds.

Theorem 53. (Polygonal line). Let $x_{k} \in[a, b]$ for $k=0,1, \ldots, n$ and let $p_{i} \in \mathbb{R}(i=0, \ldots, n)$, then the polygonal line in the following form

$$
\psi(x)=\sum_{k=0}^{n} p_{k}\left|x-x_{k}\right|
$$

is a general convex with contact function (for some continuous function $g$ : $\left.f(I)^{2} \rightarrow \mathbb{R}\right)$ if and only if the preceding coeficients satisfies the following condition

$$
2 p_{j+1}-\left(\sum_{i=0}^{j} p_{i}-\sum_{i=j+1}^{n} p_{i}\right) \geq 0 \quad(j=0, \ldots, n-2) .
$$

We notice that condition (I'), de facto, equality with condition (I) in the preceding Theorem 52. Only, in this case, we begin the proof in points $x_{j}<x_{j+1}<x_{j+2}$ for $(j=0, \ldots, n-2)$. If $p_{k} \geq 0(k=1, \ldots, n-1)$ in (42), then $\psi(x)$ is a convex function, as and reverse, if $\psi(x)$ is a convex function, then $p_{k} \geq 0(k=1, \ldots, n-1)$. 
9.2. Groupoidity of general convexity. In this section we considered, in connection with the preceding results, the following question: when the following statement holds

$$
f \in G K(I) \quad \text { implies } \quad \text { Af } \in G K(I)
$$

for every function $f$ and a linear operator $A$, where $G K(I)$ denoted the set of all continuous general convex with contact functions (for some continuous function $\left.g: f(I)^{2} \rightarrow \mathbb{R}\right)$ on $I:=[a, b]$.

In this sense, we shall denote by $C[a, b]$ a set of all functions continuous on the segment $[a, b](-\infty<a<b<+\infty)$ and by $S(D)$ one of the normed subspaces of the space all real functions defined on $D \subset \mathbb{R}$. It is obvious from the foregoing that the inclusion $G K(I) \subset C[a, b]$ is valid (because we consider only those general convex functions which are continuous). Let $\omega(t, c):=|t-c|$. We continue with the following fact.

Theorem 54. (Groupoidity of operators). Let us assume that the operator $A: C[a, b] \rightarrow S(D)$ is linear and continuous. If real numbers $p_{k}(k=$ $0,1, \ldots, m)$ satisfies inequality $\sum_{k=0}^{m} p_{k} \geq 0$, then the following statement

$$
f \in G K(I) \text { implies } \quad \text { Af } \in G K(I),
$$

for every function $t \mapsto f(t)$ is valid if and only if the following fact holds

$$
A \omega(t, c) \in G K(I) \quad \text { for every } \quad c \in I .
$$

Proof. Sufficiency. Statement (43) holds for any continuous general convex function $f$ (for some continuous function $g: f(I)^{2} \rightarrow \mathbb{R}$ ) on interval $I$. Since $\omega(t, c) \in G K(I)$ for every $c \in I$ on the basis of statement (43) the validity of (44) follows.

Necessity. Let us assume that condition (44) is valid and let us prove that the statement (43) holds true, for every function $f \in G K(I)$. If $f \in G K(I)$, then on the basis of Theorem 52, there exist $t_{k} \in I$ and $p_{k} \in \mathbb{R}(k=$ $0,1, \ldots, n)$ with property (I) such that the sequence $\left\{\psi_{n}\right\}_{n \in \mathbb{N}}$ of the form (S) satisfies condition $\left\|\psi_{n}-f\right\| \rightarrow 0(n \rightarrow \infty)$. Since $A$, by the assumptions of the statement, is a continuous operator, we have the following relation that

$$
\left\|A \psi_{n}-A f\right\| \rightarrow 0 \quad(n \rightarrow \infty)
$$

is valid for every function $f \in G K(I)$. On the other hand, since the operator $A$ is linear, in virtue of $(\mathrm{S})$ we have

$$
A \psi_{n}=\sum_{k=0}^{n} p_{k} A \omega\left(t, t_{k}\right) .
$$


Also, since $A \omega(t, c) \in G K(I)$ for every $c \in I$, thus we obtain, from Theorem 52 with condition (I), that

$$
A \omega(t, c)=\lim _{n \rightarrow \infty} \sum_{k=0}^{n} p_{k} \omega\left(t, t_{k}\right)
$$

for every $c \in I$. To multiply (46) with $p_{k}$ and to sum left and right sides, from (45), (46) and Theorem 52, we obtain

$$
A \psi_{m}=\left(\sum_{k=0}^{m} p_{k}\right) \lim _{n \rightarrow \infty} \sum_{k=0}^{n} p_{k} \omega\left(t, t_{k}\right)=\left(\sum_{k=0}^{m} p_{k}\right) A \omega(t, c) .
$$

Since $\sum_{k=0}^{m} p_{k} \geq 0,(47)$ means that $\left\{A \psi_{m}\right\}_{m \in \mathbb{N}}$ is a sequence of general convex with contact functions (for some continuous function $g: f(I)^{2} \rightarrow$ $\mathbb{R})$ for every $m \in \mathbb{N}$. From Lemma 5 and the preceding facts we have Af $\in G K(I)$ for every general convex with contact function $f$ (for some continuous function $\left.g: f(I)^{2} \rightarrow \mathbb{R}\right)$. This means that (43) holds. The proof is complete.

It is well known that a large number of theorems from the theory of convex functions are of the form $A f \geq 0$ where $x \mapsto f(x)$ is a convex function and $A$ is a given linear operator. Also this holds and for a large number of theorems from the theory of general convex functions. In this sense, directly from the preceding proof of Theorem 54, we obtain the following statement.

Theorem 54a. (Positivity of linear operators). Let us assume that the operator $A: C[a, b] \rightarrow S(D)$ is linear and continuous. If real numbers $p_{k}(k=0,1, \ldots, m)$ satisfies inequality $\sum_{k=0}^{m} p_{k} \geq 0$, then the following statement

$$
f \in G K(I) \text { implies } \text { Af } \geq 0,
$$

for every function $t \mapsto f(t)$ is valid if and only if the following inequality holds

$$
A \omega(t, c) \geq 0 \quad \text { for every } \quad c \in I \text {. }
$$

10. Miscellaneous Results, problems, and applications

10.1. (J e n s e n, [1905]). Let $D \subset \mathbb{R}^{n}$ be a convex and open set. If $f: D \rightarrow \mathbb{R}$ is a J-convex function, then for every $n \in \mathbb{N}$ and for all $x_{1}, \ldots, x_{n} \in D$ the following inequality holds

$$
f\left(\frac{x_{1}+\cdots+x_{n}}{n}\right) \leq \frac{f\left(x_{1}\right)+\cdots+f\left(x_{n}\right)}{n} .
$$

Proof. It follows from inequality $(\mathrm{J})$ by mathematical induction that for every $p \in \mathbb{N}$ and for all $x_{1}, \ldots, x_{m} \in D\left(m:=2^{p}\right)$ the following inequality holds in the form as

$$
f\left(\frac{1}{2^{p}} \sum_{i=1}^{2^{p}} x_{i}\right) \leq \frac{1}{2^{p}} \sum_{i=1}^{2^{p}} f\left(x_{i}\right) .
$$


Further, fix an $n \in \mathbb{N}$, and choose a $p \in \mathbb{N}$ such that $n<2^{p}$. Take arbitrary $x_{1}, \ldots, x_{n} \in$ $D$ and put

$$
x_{k}=\frac{1}{n} \sum_{i=1}^{n} x_{i} \quad \text { for } \quad k=n+1, \ldots, 2^{p} ;
$$

thus, since $D$ is a convex set, the points of the form (50) belong to $D$, which means that

$$
f\left(\frac{1}{n} \sum_{i=1}^{n} x_{i}\right)=f\left(\frac{1}{2^{p}} \sum_{i=1}^{2^{p}} x_{i}\right) \leq \frac{1}{2^{p}}\left[\sum_{i=1}^{n} f\left(x_{i}\right)+\left(2^{p}-n\right) f\left(\frac{1}{n} \sum_{i=1}^{n} x_{i}\right)\right],
$$

i.e., we obtain (48). The proof is complete. (For a different proof of the preceding proof see: Tas ković [2001].)

Let $D \subset \mathbb{R}^{n}$ be a convex and open set. If $f: D \rightarrow \mathbb{R}$ is a J-convex function, then for every $x, y \in D$, and for every $\lambda \in \mathbb{Q} \cap[0,1]$ the following inequality holds in the form as

$$
f(\lambda x+(1-\lambda) y) \leq \lambda f(x)+(1-\lambda) f(y) .
$$

Proof. Let $\lambda=k / n$ for every $n \in \mathbb{N}$, where $0<k<n$. If we put $x_{1}=\cdots=x_{k}=x$ and $x_{k+1}=\cdots=x_{n}=y$, then by (48) we obtain the following inequality in the form as

$$
f\left(\frac{k x+(n-k) y}{n}\right) \leq \frac{k f(x)+(n-k) f(y)}{n}
$$

which is the same as the inequality (51). If in the preceding case $\lambda=0$ or $\lambda=1$, then the inequality (51) is trivial. The proof is complete.

Annotation. If $f: D \rightarrow \mathbb{R}$ is a J-convex function and a continuous function, then (51) holds for all real $\lambda \in[0,1]$. The converse statement is also true, in the following sense, as:

Proposition 11. Let $D \subset \mathbb{R}^{n}$ be a convex and open set, and let $f: D \rightarrow \mathbb{R}$, be a function. The function $f$ is J-convex and continuous if and only if it satisfies inequality (51) for all $x, y \in D$ and for all $\lambda \in[0,1]$.

10.2. (J e n s e n, [1905]). Let $D \subset \mathbb{R}^{n}$ be a convex set, and let $F \subset \mathbb{R}$ be a field. If a function $f: D \rightarrow[-\infty,+\infty)$ satisfies (51) for all $\lambda \in \mathbb{Q} \cap[0,1]$, then the following inequality holds in the from as

$$
f\left(\sum_{i=1}^{n} \lambda_{i} x_{i}\right) \leq \sum_{i=1}^{n} \lambda_{i} f\left(x_{i}\right)
$$

for every $n \in \mathbb{N}$, for all $x_{1}, \ldots, x_{n} \in D$, and for all $\lambda_{1}, \ldots, \lambda_{n} \in F \cap[0,1]$ such that $\lambda_{1}+\cdots+\lambda_{n}=1$.

Proof. The proof runs by induction. For $n=2$ inequality (52) is identical with (51). Now suppose (52) to be true for an $n \in \mathbb{N}$. Take arbitrary $x_{1}, \ldots, x_{n+1} \in D$ and $\lambda_{1}, \ldots, \lambda_{n+1} \in$ $F \cap[0,1]$ such that $\lambda_{1}+\cdots+\lambda_{n+1}=1$. If $\lambda_{1}=\cdots=\lambda_{n}=0, \lambda_{n+1}=1$, then (52) is triviaL. If $\lambda_{1}+\cdots+\lambda_{n} \neq 0$, then we get by $(51)$

$$
\begin{array}{r}
f\left(\sum_{i=1}^{n+1} \lambda_{i} x_{i}\right)=f\left(\left(\sum_{i=1}^{n} \lambda_{i}\right) \frac{\lambda_{1} x_{1}+\cdots+\lambda_{n} x_{n}}{\lambda_{1}+\cdots+\lambda_{n}}+\lambda_{n+1} x_{n+1}\right) \leq \\
\leq\left(\sum_{i=1}^{n} \lambda_{i}\right) f\left(\frac{\lambda_{1}}{\lambda_{1}+\cdots+\lambda_{n}} x_{1}+\cdots+\frac{\lambda_{n}}{\lambda_{1}+\cdots+\lambda_{n}} x_{n}\right)+\lambda_{n+1} f\left(x_{n+1}\right),
\end{array}
$$


and thus, from all the preceding facts, and by the induction hypothesis, we obtain the following inequality in the form as

$$
\begin{aligned}
& f\left(\frac{\lambda_{1}}{\lambda_{1}+\cdots+\lambda_{n}} x_{1}+\cdots+\frac{\lambda_{n}}{\lambda_{1}+\cdots+\lambda_{n}} x_{n}\right) \leq \\
\leq & \frac{\lambda_{1}}{\lambda_{1}+\cdots+\lambda_{n}} f\left(x_{1}\right)+\cdots+\frac{\lambda_{n}}{\lambda_{1}+\cdots+\lambda_{n}} f\left(x_{n}\right),
\end{aligned}
$$

and thus, from (53), we obtain (52) for $n+1$. This means that inequality (52) is generally true. The proof is complete.

Annotation. We notice that inequality (52) with arbitrary $\lambda_{1}, \ldots, \lambda_{n} \in \mathbb{Q} \cap[0,1]$, adding up to 1 , and for every continuous J-convex function $f: D \rightarrow \mathbb{R}$ with arbitrary $\lambda_{1}, \ldots, \lambda_{n} \in$ $[0,1]$, adding up to 1 .

10.3. (A characterization of J-convexity). Let $D \subset \mathbb{R}^{n}$ be a convex and open set. Show that a function $f: D \rightarrow \mathbb{R}$ is J-convex if and only if the set

$$
S:=\{(x, y) \in D \times \mathbb{R}: y>f(x)\}
$$

is J-convex. (A nonempty set $A \subset \mathbb{R}^{n}$ is called $\mathbf{J}$-convex iff $(x+y) / 2 \in A$ for all $x, y \in A$.)

Open problem 2. Show that a function $f: D \rightarrow \mathbb{R}\left(D \subset \mathbb{R}^{n}\right.$ is a convex and open set) is general J-convex if and only if the set

$$
G:=\{(x, y) \in D \times \mathbb{R}: y>\max \{f(x), g(f(x), f(x))\}\}
$$

is a J-convex set for a function $g: f(D)^{2} \rightarrow \mathbb{R}$ (for which $f$ is a general J-convex function).

10.4. (Jensen convexity on rational lines). In this section we discuss some properties of J-convex functions connected with their boundedness and continity.

Lemma 6. Let $D \subset \mathbb{R}^{n}$ be a convex and open set, and let $f: D \rightarrow \mathbb{R}$ be a J-convex function. Then the following inequalities hold in the form as

$$
\frac{f(x)-f(x-n d)}{n} \leq \frac{f(x)-f(x-m d)}{m} \leq \frac{f(x+m d)-f(x)}{m} \leq \frac{f(x+n d)-f(x)}{n}
$$

for every $x \in D$, for every $d \in \mathbb{R}^{n}$, and for all $m, n \in \mathbb{N}$ such that $0<m<n$ and $x \pm n d \in D$.

Proof. We use problem 1 (of this part of the book). Take in (48) $x_{1}=\cdots=x_{m}=x+n d$, $x_{m+1}=\cdots=x_{n}=x$. We obtain

$$
f\left(\frac{m(x+n d)+(n-m) x}{n}\right) \leq \frac{m f(x+n d)+(n-m) f(x)}{n},
$$

i.e., $f(x)-f(x-m d) \leq f(x+m d)-f(x)$, and the middle inequality in (54) results on dividing both the sides by $m$. The proof is complete.

Lemma 7. Let $D \subset \mathbb{R}^{n}$ be a convex and open set, and let $f: D \rightarrow \mathbb{R}$ be a J-convex function. If $f$ is bounded above on a set $A \subset D$, then it is also bounded above (by the same constant) on $Q(A)$, where $Q(A)$ is the convex hull of $A$.

Proof. Suppose that for a certain constant $M \in \mathbb{R}$ we have $f(t) \leq M$ for $t \in A$. Take an arbitrary $x \in Q(A)$. Then there exist an $n \in \mathbb{N}$, and $t_{1}, \ldots, t_{n} \in A$, and $\lambda_{1}, \ldots, \lambda_{n} \in$ $\mathbb{Q} \cap[0,1]$ such that

$$
x=\lambda_{1} t_{1}+\cdots+\lambda_{n} t_{n} \quad\left(\lambda_{1}+\cdots+\lambda_{n}=1\right) ;
$$


thus, by problems 2 and 3, we obtain from the preceding facts that the following inequalities hold in the form as

$$
f(x) \leq \sum_{i=1}^{n} \lambda_{i} f\left(t_{i}\right) \leq \sum_{i=1}^{n} \lambda_{i} M=M \sum_{i=1}^{n} \lambda_{i}=M,
$$

which means that $f$ is a bounded above function by $M$ on the set $Q(A)$. The proof is complete.

Corollary 9. Let $D \subset \mathbb{R}^{n}$ be a convex and open set, and let $f: D \rightarrow \mathbb{R}$ be a J-convex function. If $f$ is bounded above on a set $A \subset D$, then it is also bounded above (by the same constant) on $J(A)$, where $J(A)$ is the J-convex hull of $A$.

Proof. This could be proved by the same argument as problem 1, but we will derive this directly from Lemma 7 . Since $1 / 2 \in \mathbb{Q}$, the set $Q(A)$ is J-convex, and hence $J(A) \subset Q(A)$. So our corollary is an immediate consequence of Lemma 7 . The proof is complete.

Corollary 10. Let $D \subset \mathbb{R}^{n}$ be a convex and open set, and let $f: D \rightarrow \mathbb{R}$ be a J-convex function. For arbitrary $x, y \in D$, the function $f$ is bounded above on the rational segment $Q(x, y){ }^{4}$

Proof. This follows from Lemma 7 in view of the fact that every function is bounded on every finite set. The proof is comeplete.

Lemma 8. Let $D \subset \mathbb{R}^{n}$ be a convex and open set, and let $f: D \rightarrow \mathbb{R}$ be a J-convex function. Then, for arbitrary $x, y \in D$, the function $f$ is bounded below on the rational segment $Q(x, y)$.

Proof. Take arbitrary $x, y \in D$ and $t \in Q(x, y)$. According to (55) there exists a $\lambda \in$ $\mathbb{Q} \cap[0,1]$ such that $t=\lambda x+(1-\lambda) y$. Moreover, by Corollary 9 , there exists a constant $M$ such that

$$
f(s) \leq M \quad \text { for every } \quad s \in Q(x, y) .
$$

Put $u=(x+y) / 2$ and $v=2 u-t$. Then, $v=x+y-\lambda x-(1-\lambda) y=\lambda y+(1-\lambda) x \in$ $Q(x, y)$, whence by $(56) f(v) \leq M$. Also, we have $2 f(u) \leq f(v)+f(t)$, whence

$$
f(t) \geq 2 f(u)-f(v) \geq 2 f(u)-M
$$

thus, this means that $f$ is a bounded below function on $Q(x, y)$ by the constant $2 f(u)-M$. The proof is complete. (This constant, i.e., $2 f(u)-M$ depends on $x$ and $y$, but not on a particular $t \in Q(x, y)$ !)

Theorem 55. (Uniformly continuous). Let $D \subset \mathbb{R}^{n}$ be a convex and open set, and let $f: D \rightarrow \mathbb{R}$ be a J-convex function. Then, for arbitrary $a, b \in D$, the function $f \mid Q(a, b)$ is uniformly continuous.

\footnotetext{
${ }^{4}$ Rational segments. If $a, b \in \mathbb{R}^{n}$ are arbitrary points, we write $Q(a, b)$ insteaed of $A(\{a, b\})$, and call $Q(a, b)$ the rational segment joining the points $a, b$. It is easily seen that

$$
Q(a, b):=\left\{x \in \mathbb{R}^{n}: x=\lambda a+(1-\lambda) b, \quad \lambda \in \mathbb{Q} \cap[0,1]\right\},
$$

for if in the combination $x=\alpha_{1} a_{1}+\cdots+\alpha_{n} a_{n}$ several $a_{i}$ 's equal $a$, and the remaining equal $b$, we can make the suitable reduction, denoting the sum of the coefficients of $a_{i}=a$ by $\lambda$. And if one of $a, b$ is laching in the combination, we can always add it with the coefficient zero!
} 
We notice that every uniformly continuous function on a set $A \subset \mathbb{R}^{n}$ can be uniquely extended onto $\mathrm{ClA}$ to a continuous function, so we obtain from Theorem 55 the following fact.

Theorem 56. Let $D \subset \mathbb{R}^{n}$ be a convex and open set, and let $f: D \rightarrow \mathbb{R}$ be a J-convex function. Then, for arbitrary $a, b \in D$, there exists a unique continuous function $g_{a b}$ : $C l Q(a, b) \rightarrow \mathbb{R}$ such that $g_{a b}|Q(a, b)=f| Q(a, b)$. The function $g_{a b}$ for all $x, y \in C l Q(a, b)$ satisfies

$$
g_{a b}\left(\frac{x+y}{2}\right) \leq \frac{g_{a b}(x)+g_{a b}(y)}{2} .
$$

10.5. (Local boundedness of J-convex functions). Let $D \subset \mathbb{R}^{n}$. A function $f: D \rightarrow$ $\mathbb{R}$ is called locally bounded (locally bounded above, locally bounded below) at a point $x_{0} \in D$ iff there exists a neighbourhood $U \subset D$ of $x_{0}$ such that the function $f$ is bounded (bounded above, bounded below) on $U$. The following three statements refer to the local boundedness of $f$ on open subsets of $D$ :

(a) Let $D \subset \mathbb{R}^{n}$ be a convex and open set, and let $f: D \rightarrow \mathbb{R}$ be a J-convex function. If $f$ is locally bounded above at a point $x_{0} \in D$, then it is locally bounded above at every point $x \in D$.

(b) Let $D \subset \mathbb{R}^{n}$ be a convex and open set, and let $f: D \rightarrow \mathbb{R}$ be a J-convex function. If $f$ is locally bounded below at a point $x_{0} \in D$, then it is locally bounded below at every point $x \in D$.

(c) Let $D \subset \mathbb{R}^{n}$ be a convex and open set, and let $f: D \rightarrow \mathbb{R}$ be a J-convex function. If $f$ is locally bounded above at a point $x_{0} \in D$, then it is locally bounded at every point $x \in D$.

Proof for (c). By (a) $f$ is locally bounded above at every point $x \in D$, and so if we show that $f$ is locally bounded below at $x_{0}$, then it will follow by (b) that is locally bounded below, and hence locally bounded at every point $x \in D$.

Let $K \subset D$ be an open ball around $x_{0}$ such that $f$ is bounded above on $K$, i.e., $f(t) \leq M$ for $t \in K$, with a real constant $M$. Take an arbitrary $u \in K$ and put $t=2 x_{0}-u$ so that $2 x_{0}=u+t$. We have $t-x_{0}=-\left(u-x_{0}\right)$, whence $\left|t-x_{0}\right|=\left|u-x_{0}\right|$, which shows that $t \in K$. We have $2 f\left(x_{0}\right)=f(u)+f(t)$, whence by the preceding we obtain

$$
f(u) \geq 2 f\left(x_{0}\right)-f(t) \geq 2 f\left(x_{0}\right)-M ;
$$

thus, $f$ is a bounded below function on $K$ by the constant $2 f\left(x_{0}\right)-M$. The proof is complete.

10.6. (The lower hull of J-convex function). Let $D \subset \mathbb{R}^{n}$ be an open set, and let $f: D \rightarrow \mathbb{R}$ be a function. For $x \in D$ and $r>0$ sufficiently small (such that $K(x, r) \subset D$ ) the function $m_{f}: D \rightarrow[-\infty,+\infty)$ is called the lower hull of $f$, and its value at an $x \in D$ is called the infimum of $f$ at $x$, i.e.,

$$
m_{f}(x)=\lim _{r \rightarrow 0+} \inf _{K(x, r)} f ;
$$

and similarly, we can define the function $M_{f}: D \rightarrow(-\infty,+\infty]$ in the following adequate form as

$$
M_{f}(x):=\lim _{r \rightarrow 0+} \sup _{K(x, r)} f,
$$

which is called an upper hull of $f$, and its value at an $x \in D$ is called the supremum of $f$ at $x$.

If the set $D$ is convex, if the function $f$ is J-convex, and if $f$ is locally bounded below at a point $x_{0} \in D$, then the infimum of $f$ at every $x \in D$ is finite. Thus, in such a case, the function $m_{f}$ is finite, i.e., $m_{f}: D \rightarrow \mathbb{R}$. In the other case, $f$ is locally unbounded below at every point $x \in D$, which means that $m_{f}(x)=-\infty$ for every $x \in D$. Similarly, 
it follows, from the preceding problem 6 , that either $M_{f}(x)$ is finite for every $x \in D$, i.e., $M_{f}: D \rightarrow \mathbb{R}$ or $M_{f}(x)=+\infty$ for every $x \in D$.

Theorem 57. Let $D \subset \mathbb{R}^{n}$ be a convex and open set, and let $f: D \rightarrow \mathbb{R}$ be a J-convex function. If $m_{f} \neq-\infty$, then the function $m_{f}: D \rightarrow \mathbb{R}$ is continuous and J-convex.

10.7. (Theorem of Bernstein-Doetsch). Let $D \subset \mathbb{R}^{n}$ be an open set, and let $f: D \rightarrow$ $\mathbb{R}$ be a function. If follows directly from the definition of the lower hull of $f$ that the following inequality holds in the from as

$$
m_{f}(x) \leq f(x) \text { for } x \in D,
$$

but, if the function $f$ is $\mathrm{J}$-convex and if the set $D$ is convex, we have more precise information in the following form:

(a) Let $D \subset \mathbb{R}^{n}$ be a convex and open set, and let $f: D \rightarrow \mathbb{R}$ be a J-convex function. If at a point $\xi \in D$ we have $f(\xi) \neq m_{f}(\xi)$, then $f$ is not locally bounded at $\xi$.

(b) (B e r n ste i n-D o et s ch [1915]). Let $D \subset \mathbb{R}^{n}$ be a convex and open set, and let $f: D \rightarrow \mathbb{R}$ be a J-convex function. If $f$ is locally bounded above at a point of $D$, then it is continuous in $D$.

Proof for (b). Let $f$ be locally bounded above at a point of $D$. By problem $6 f$ is locally bounded at every point of $D$, whence it follows in virtue of (a) that $f(x)=m_{f}(x)$ for $x \in D$. Since $f$ is locally bounded, we have $m_{f} \neq-\infty$, whence, by problem $7, m_{f}$ is continuous in $D$. Consequently $f$ is continuous in $D$.

Since every open set is a neighborhood of any of its points, we get hence the following consequence in the form as:

Corollary 11. Let $D \subset \mathbb{R}^{n}$ be a convex and open set, and let $f: D \rightarrow \mathbb{R}$ be a J-convex function. If $f$ is bounded above on a nonempty open set $U \subset D$, then it is continuous in D.

If a function is continuous at a point, then it is locally bounded at this point. Thus Bernstein-Doetsch theorem implies the following statement.

Theorem 58. Let $D \subset \mathbb{R}^{n}$ be a convex and open set, and let $f: D \rightarrow \mathbb{R}$ be a Jconvex function. Then, either $f$ is continuous in $D$ or $f$ is totally discontinuous (i.e., discontinuous at every point of $D$ ) in $D$.

Let $D \subset \mathbb{R}^{n}$ be a convex and open set, and let $f: D \rightarrow \mathbb{R}$ be a J-convex function. Let $x \in D$ and $y \in \mathbb{R}^{n}$ be such that $x+y \in D$. Prove that

$$
\lim _{n \rightarrow \infty} f\left(x+\frac{y}{2^{n}}\right)=f(x) .
$$

Open problem 3. Let $D \subset \mathbb{R}^{n}$ be a convex and open set. If $f: D \rightarrow \mathbb{R}$ is a general J-convex function, does equality (57) hold?!

Let $D \subset \mathbb{R}^{n}$ be a convex and open set, and let $f: D \rightarrow \mathbb{R}$ be a J-convex function. Let $0, a, b, a+b \in D$. Prove that the set

$$
A:=\left\{x \in \mathbb{R}^{n}: x=\lambda a+\eta b ; \quad \lambda, \eta \in \mathbb{Q} \cap[0,1]\right\}
$$

is contained in $D$. Also, prove that the function $f$ is bounded on the set $A$. (Does this statement holds whenever $f: D \rightarrow \mathbb{R}$ is a general J-convex function?)!

Instruction for proof. Clearly $f$ is bounded above on $A$, and bounded below on $Q(0, a+$ $b)$. For any $x \in A, x=\lambda a+\eta b$ and $\lambda, \eta \in \mathbb{Q} \cap[0,1]$ consider the points $y=\eta a+\lambda b$ and $2 z=x+y$ ! 
Let $D \subset \mathbb{R}^{n}$ be a convex and open set, and let $f: D \rightarrow \mathbb{R}$ be a J-convex function. Let $0, a, b, a+b \in D$, and let $\lambda_{n}, \mu_{n} \in \mathbb{Q} \cap[0,1]$ for $n \in \mathbb{N}$ such that $\lim _{n \rightarrow \infty} \lambda_{n}=\lambda$ and $\lim _{n \rightarrow \infty} \mu_{n}=\mu$. Prove that if $\lambda, \mu \in \mathbb{Q} \cap[0,1]$, then

$$
\lim _{n \rightarrow \infty} f\left(\lambda_{n} a+\mu_{n} b\right)=f(\lambda a+\mu b) .
$$

In as much $0, a, b \in D$ and $\lambda_{n}, \mu_{n} \in \mathbb{Q}(n \in \mathbb{N})$ such that $\lim _{n \rightarrow \infty} \lambda_{n}=\lambda, \lim _{n \rightarrow \infty} \mu_{n}=$ $\mu, \lambda a+\mu b \in D, \lambda_{n} a+\mu_{n} b \in D(n \in \mathbb{N})$ and $\lambda, \mu \in \mathbb{Q}$, then equality (58) holds also.

Let $D \subset \mathbb{R}^{n}$ be a convex and open set, and let $f: D \rightarrow \mathbb{R}$ be a J-convex function. Let $0, a, b \in D$. Prove that if $a$ and $b$ are linearly independent over $\mathbb{R}$, then the function $f \mid E(a, b) \cap D$ is continuous, where the set $E(a, b)$ is called the rational plane passing through the origin, $a$ and $b$.

Annotation. We notice that this statement is not hold if $a, b \in \mathbb{R}$ are lineraly dependent over $\mathbb{R}$. (Take incommensurable $a, b \in \mathbb{R}$, and let $f: \mathbb{R} \rightarrow \mathbb{R}$ be an additive function such that $f(a)=1$ and $f(b)=0$. Consider a sequence $x_{n}=p_{n} a+q_{n} b\left(p_{n}, q_{n} \in \mathbb{Z}\right.$ for $\left.n \in \mathbb{N}\right)$ such that $\lim _{n \rightarrow \infty} x_{n}=0$ !).

10.8. (The equality of lower and upper hull). Let $D \subset \mathbb{R}^{n}$ be an open set, and let $f: D \rightarrow \mathbb{R}$ be a function. Prove that the condition $M_{f}(x)=m_{f}(x)$ is necessary and sufficient for the continuity of $f$ at $x \in D$.

(C s á s zá r, [1958]). Let $D \subset \mathbb{R}^{n}$ be a convex and open set, and let $f: D \rightarrow \mathbb{R}$ be a J-convex function. Let $T \subset D$ be a measurable set such that $m(T)>0$. Then for no measurable function $g: T \rightarrow \mathbb{R}$ we may have

$$
m_{f}(x)<g(x) \leq f(x) \text { for every } \quad x \in T .
$$

(C s ás z ár, [1958]). Let $D \subset \mathbb{R}^{n}$ be a convex and open set, and let $f: D \rightarrow \mathbb{R}$ be a J-convex function. Let $T \subset D$ be a set fulfilling one of the following conditions: $T$ is a Lebesgue measurable set of positive measure or $T$ is of the second category and with Baire property. Then cannot hold for any real constant $K$ the following inequalities of the form

$$
m_{f}(x)<K \leq f(x) \text { for every } \quad x \in T .
$$

(H u k u h a r a, [1954]). Let $D \subset \mathbb{R}^{n}$ be a convex and open set, and let $T \subset D$ be a set fulfilling one of conditions: $T$ is a Lebesgue measurable set of positive measure or $T$ is of the second category and with the Baire property. Then $T \in \mathcal{F}(D)$, where $\mathcal{F}(D)$ is the class of all sets $T \subset D$ with the property that every J-convex function $f: D \rightarrow \mathbb{R}$ which is bounded below on $T$ is locally bounded below at every poit of $D$.

We notice that $\mathrm{H} \mathrm{u} \mathrm{k} \mathrm{u} \mathrm{h} \mathrm{a} \mathrm{r} \mathrm{a} \mathrm{[1954]} \mathrm{proved} \mathrm{the} \mathrm{preceding} \mathrm{result} \mathrm{in} \mathrm{the} \mathrm{case} n=1$. This result has then be extended to higher dimensions by M a r c u s [1959] and C s á s zár [1958].

(S m i t a l, [1976]). Let $D \subset \mathbb{R}^{n}$ be a convex and open set, and let $\mathcal{U}$ denoted the sets $T \subset \mathbb{R}^{n}$ such that every J-convex function $f: D \rightarrow \mathbb{R}$ bounded above on $T$ is continuous in $D$. Then $\mathcal{F}(D) \subset \mathcal{U}$, where $\mathcal{F}(D)$ is defined in the preceding problem 16 .

Proof. Let $T \in \mathcal{F}(D)$, and let $f: \mathbb{R}^{n} \rightarrow \mathbb{R}$ be an arbitrary additive function bounded above on $T: f(x) \leq M$ for $x \in T$. Put $g=-f \mid D$. Then $g: D \rightarrow \mathbb{R}$ is a J-convex function bounded below on $T$ i.e.,

$$
g(x) \geq-M \quad \text { for every } \quad x \in T,
$$

thus, since $T \in \mathcal{F}(D)$, this means that $g$ is locally bounded below at every point of $D$, and hence $f$ is locally bounded above at every point of $D$. Applying BernsteinDoetsch theorem (problem 8) we obtain that $f$ is continuous. Hence $T \in \mathcal{U}$. The proof is complete. 
Annotation. But actually the inclusion $\mathcal{F}(D) \subset \mathcal{U}$ cannot be replaced by the equality, as may be seen from the example in: K u c z m a [1970].

10.9. (Jensen equation, $\mathrm{K} \mathrm{u} \mathrm{c} \mathrm{z} \mathrm{m} \mathrm{a} \mathrm{[1970]).} \mathrm{The} \mathrm{equation} \mathrm{resulting} \mathrm{on} \mathrm{replacing} \mathrm{in}$ the Jensen inequality $(\mathrm{J})$ the sign of inequality by that of equality in the form as

$$
f\left(\frac{x+y}{2}\right)=\frac{f(x)+f(y)}{2}
$$

is known as the Jensen equation by A c z él [1966]. We will consider equation (59) for functions $f: D \rightarrow \mathbb{R}$, where $D \subset \mathbb{R}^{n}$ is a convex set.

If $D$ were also open, then $f$ satisfying (59) would be J-convex, and thus all the results established in the previous sections of this book for J-convex functions would apply. The following facts hold:

(a) Let $D \subset \mathbb{R}^{n}$ be a convex set such that $0 \in D$, and let $f: D \rightarrow \mathbb{R}$ be a solution of equation (59) such that $f(0)=0$. Then, for every $x \in D$ and $n \in \mathbb{N}$,

$$
f\left(\frac{x}{2^{n}}\right)=\frac{1}{2^{n}} f(x) .
$$

(b) Let $D \subset \mathbb{R}^{n}$ be a convex set such that $\operatorname{int}(D)$ is nonempty, and let $f: D \rightarrow \mathbb{R}$ be a solution of equation (59). Fix an $x_{0} \in \operatorname{int}(D)$, and define the function $g: D \backslash\left\{x_{0}\right\} \rightarrow \mathbb{R}$ by $g(x)=f\left(x_{0}+x\right)-f\left(x_{0}\right)$. Then there exists a unique function $\mu: \mathbb{R}^{n} \rightarrow \mathbb{R}$ satisfying equation (59) in $\mathbb{R}^{n}$ and such that

$$
\mu(x)=g(x) \quad \text { for every } \quad x \in D \backslash\left\{x_{0}\right\} .
$$

(c) Let a function $f: \mathbb{R}^{n} \rightarrow \mathbb{R}$ satisfy equation (59) and the relation of the form as $f(0)=0$. Then $f$ is an additive function.

(d) Let $D \subset \mathbb{R}^{n}$ be a convex set such that $\operatorname{int}(D)$ is a nonempty set, and let $f: D \rightarrow \mathbb{R}$ be a solution of equation (59). Then there exist an additive function $g: \mathbb{R}^{n} \rightarrow \mathbb{R}$ and a constant $a \in \mathbb{R}$ such that

$$
f(x)=g(x)+a \quad \text { for every } \quad x \in D .
$$

(e) Let $D \subset \mathbb{R}^{n}$ be a convex set such that $\operatorname{int}(D)$ is a nonempty set. A function $f: D \rightarrow \mathbb{R}$ is a continuous solution of equation (59) if and only if with certain constants $c \in \mathbb{R}^{n}$ and $a \in \mathbb{R}$ is

$$
f(x)=c x+a \quad \text { for every } \quad x \in D .
$$

(f) Let $D \subset \mathbb{R}^{n}$ be a convex set such that $\operatorname{int}(D)$ is a nonempty set, and let $f: D \rightarrow \mathbb{R}$ be a solution of equation (59). If $f$ is measurable or is bounded above (or below) on a set $T \in \mathcal{U}$, then $f$ is continuous.

10.10. (Strong J-convexity, P o l z a k [1966]). Let $I:=(a, b) \subset \mathbb{R}$ be an open interval. Call that the function $f:(a, b) \rightarrow \mathbb{R}$ is strongly J-convex if there is an $\alpha>0$ such that

$$
f\left(\frac{x+y}{2}\right) \leq \frac{f(x)+f(y)}{2}-\frac{1}{4} \alpha(x-y)^{2}
$$

for all $x, y \in(a, b)$. Then $f$ is an general J-convex function. Show that this is equivalent to each of the following conditions:

(a) The following inequality holds in the form for all points $x, y \in I$ and for arbitrary $\lambda \in[0,1]$ as

$$
f(\lambda x+(1-\lambda) y) \leq \lambda f(x)+(1-\lambda) f(y)-\lambda(1-\lambda) \alpha(x-y)^{2} .
$$

(b) For each $x_{0} \in(a, b)$ there is a linear function $A$ such that the following inequality holds for every $x \in(a, b)$ in the form as

$$
f(x) \geq f\left(x_{0}\right)+A\left(x-x_{0}\right)+\alpha\left(x-x_{0}\right)^{2} .
$$


(c) For the differentiable function $f:(a, b) \rightarrow \mathbb{R}$ the following inequality holds for all points $x, y \in(a, b)$ in the form as

$$
\left[f^{\prime}(x)-f^{\prime}(y)\right](x-y) \geq 2 \alpha(x-y)^{2} .
$$

(d) For the differentiable function $f:(a, b) \rightarrow \mathbb{R}$ the following inequality holds for all points $x, x_{0} \in(a, b)$ in the form as

$$
f(x) \geq f\left(x_{0}\right)+f^{\prime}(x)\left(x-x_{0}\right)+\alpha\left(x-x_{0}\right)^{2} .
$$

(e) For the twice differentiable function $f:(a, b) \rightarrow \mathbb{R}$ the following inequality holds for every point $x \in(a, b)$ in the form as $f^{\prime \prime}(x) \geq 2 \alpha$.

Annotation. If $f$ is strongly J-convex on $(a, b)$, then $f$ is bounded below, $\{x: f(x)<\lambda\}$ is bounded for arbitrary $\lambda$, and $f$ has a unique minimum on any closed subinterval of $(a, b)$. What else is true? Generalize the whole idea to the setting of a normed linear space. See: Polzak [1966], Lyubich and M a istrovskij [1970], Roberts-Varberg [1973] and Tasković [2005].

10.11. (Uniform J-convexity, L e v i t a n-P o l z a k [1966]). Let $\mathcal{U}$ be an open convex set in a normed linear space $L$. Call $f: \mathcal{U} \rightarrow \mathbb{R}$ uniformly J-convex if there is an increasing function $\delta: \mathbb{R}_{+}^{0} \rightarrow \mathbb{R}_{+}^{0}$ with $\delta(0)=0$ and $\delta(t)>0$ for $t>0$ such that

$$
f\left(\frac{x+y}{2}\right) \leq \frac{f(x)+f(y)}{2}-\delta(\|x-y\|)
$$

for all $x, y \in \mathcal{U}$. On the other hand, the function $f: \mathcal{U} \rightarrow \mathbb{R}$ is uniformly J-quasiconvex if the following inequality holds in the form as

$$
f\left(\frac{x+y}{2}\right) \leq \max \{f(x), f(y)\}-\delta(\|x-y\|)
$$

for an increasing function $\delta: \mathbb{R}_{+}^{0} \rightarrow \mathbb{R}_{+}^{0}$ with $\delta(0)=0$ and $\delta(t)>0$ for $t>0$ and for all $x, y \in \mathcal{U}$.

Annotation. For the develop the theory of such functions see: P o l z a k [1966], L e v itan - Polzak [1966], Lyubich - Maistrovskij [1970], and Tasković [1994].

10.12. (General uniform J-convexity, T a s k o v i ć [1994]). Let $U$ be an open convex set in a normed linear space $L$. Call $f: U \rightarrow \mathbb{R}$ general uniformly J-convex if there exists a function $g: f(U)^{2} \rightarrow \mathbb{R}$ and a function $\sigma: \mathbb{R}_{+}^{0} \rightarrow \mathbb{R}$ such that

$$
f\left(\frac{x+y}{2}\right) \leq \max \{f(x), f(y), g(f(x), f(y))\}+\sigma(\|x-y\|)
$$

for all $x, y \in U$. Evidently, this class of functions is the biggest by breadth of the preceding classes of functions: strong J-convexity and uniform J-convexity!

10.13. (Graphs of J-convex functions). Let $D \subset \mathbb{R}^{n}$ be a convex and open set, and let $f: D \rightarrow \mathbb{R}$ be a J-convex function. Let $m_{f}$ be the lower hull of $f$. By Theorem 57 (in problem 7) either $m_{f}(x)=-\infty$ for all $x \in D$, or $m_{f}: D \rightarrow \mathbb{R}$ is a continuous and J-convex function. Let

$$
G r(f):=\left\{(x, y) \in \mathbb{R}^{n+1}: x \in D, y=f(x)\right\}
$$

be the graph of $f$. Then we have the following result: If $f: D \rightarrow \mathbb{R}$ is a discontinuous J-convex function, then the set $G r(f)$ is dense in the set of the form as

$$
A_{f}:=\left\{(x, y) \in \mathbb{R}^{n+1}: x \in D, y>m_{f}(x)\right\} .
$$


10.14. (Characterizations of convex functions). Let $D \subset \mathbb{R}^{n}$ be a convex and open set. We say that a J-convex function $f: D \rightarrow \mathbb{R}$ fulfils the following inequality of the form as

$$
f(\lambda x+(1-\lambda) y) \leq \lambda f(x)+(1-\lambda) f(y)
$$

for all $x, y \in D$ and arbitrary $\lambda \in \mathbb{Q} \cap[0,1]$. It was also pointed out that if, moreover, $f$ is continuous, then inequality (60) holds actually for all real $\lambda \in[0,1]$.

The convex function $f: D \rightarrow \mathbb{R}$ (=: J-convex and continuous) if and only if it satisfies inequality (60) for all $x, y \in D$ and for arbitrary $\lambda \in[0,1]$.

Let $J \subset \mathbb{R}$ be an open interval and let $f: J \rightarrow \mathbb{R}$ be a function. Each of the following conditions (postulated for every $x, y, z \in j$ with $x<y<z$ ) is necessary and sufficient for the function $f$ to be convex:

$$
\begin{gathered}
\frac{f(z)-f(x)}{z-x} \leq \frac{f(z)-f(y)}{z-y} \\
\frac{f(y)-f(x)}{y-x} \leq \frac{f(z)-f(x)}{z-x} \\
(z-x) f(y) \leq(y-x) f(z)+(z-y) f(x) .
\end{gathered}
$$

10.15. (Differences quotients of convex functions). Let $J \subset \mathbb{R}$ be an open interval, and let $f: J \rightarrow \mathbb{R}$ be a function. We define a function $I(x, h)$ for $x \in J$ and $h \in \mathbb{R}$ such that $h \neq 0, x+h \in J$, and

$$
I(x, h):=\frac{f(x+h)-f(x)}{h}
$$

(a) Let $J \subset \mathbb{R}$ be an open interval, and let $f: J \rightarrow \mathbb{R}$ be a convex function. Then the corresponding function $I$ defined by (64) is increasing with respect to either variable.

(b) Let $J \subset \mathbb{R}$ be an open interval, and let $f: J \rightarrow \mathbb{R}$ be a function. Let $I$ be defined by (64). If for $h>0$ the function $I$ is increasing with respect to $h$, then $f$ is convex. If $f$ is increasing with respect to $x$, then $f$ is J-convex, but need not be continuous.

(c) Let $J \subset \mathbb{R}$ be an open interval, and let $f: J \rightarrow \mathbb{R}$ be a convex function. Then, for every fixed $h>0$, the function $\triangle_{h} f(x):=f(x+h)-f(x)$ (defined for $x \in J$ such that $x+h \in J)$ is increasing.

(d) Let $J \subset \mathbb{R}$ be an open interval, $J=(a, b)$, and let $f: J \rightarrow \mathbb{R}$ be a convex function. Then either $f$ is monotonic in $J$, or there exists a point $x_{0} \in J$ such that $f$ is decreasing in $\left(a, x_{0}\right)$, and increasing in $\left(x_{0}, b\right)$.

Proof for (b). Suppose that $I$ is increasing with respect to $h$. Take arbitrary $a<b<c$ $(a, b, c \in J)$, and put $x=a, h=b-a, r=c-a>h$. Then $I(x, h) \leq I(x, r)$, i.e.,

$$
\frac{f(b)-f(a)}{b-a} \leq \frac{f(c)-f(a)}{c-a},
$$

i.e., consequently, $f$ satisfies condition (62) in problem 23, and thus it is convex.

Now let $I$ be increasing with respect to $x$. Take arbitrary $x, y \in J(x \neq y)$. thus one of $x, y$ is smaller than the other. Let $2 h=y-x, a=x, b=x+(y-x) / 2=a+h>a$. Hence $I(a, h) \leq I(b, h)$, i.e.,

$$
\frac{f\left(\frac{x+y}{2}\right)-f(x)}{\frac{y-x}{2}} \leq \frac{f(y)-f\left(\frac{x+y}{2}\right)}{\frac{y-x}{2}} .
$$

or, after multiplication by $(y-x) / 2>0$, and rearranging the remaining terms, $f$ is a $\mathrm{J}$-convex function. If $y=x$, then convexity is equivalent to the Jensen inequality $(\mathrm{J})$, Thus $f$ is J-convex function. 
The monotonicity of $I$ woth respect to $x$ does not imply the continuity of $f$. For example, if $f: \mathbb{R} \rightarrow \mathbb{R}$ is a discontinuous additive function, then the function

$$
I(x, h)=\frac{f(x+h)-f(x)}{h}=\frac{f(h)}{h}
$$

does not depend on $x$, and thus, as a function of $x$, it is constant, and hence increasing. But $f$ is discontinuous. The proof is complete.

Remark. On the other hand, we may observe that the J-convexity of $f$ alone does not imply the monotonicity of $I(x, h)$ with respect to $x$. For an example for this see: Tasković [2005].

10.16. (Restriction of general convexity). Let $D \subset \mathbb{R}^{n}$ be a convex and open set. Then the function $f: D \rightarrow \mathbb{R}$ is general convex if and only if the function $f_{x, y}:[0,1] \rightarrow \mathbb{R}$ is general convex defined by $f_{x, y}(\lambda):=f(\lambda x+(1-\lambda) y)$ for all $x, y \in D$.

Proof. Let $f: D \rightarrow \mathbb{R}$ be a general convex function. If $x, y \in D$ and $\lambda, \mu, \eta \in[0,1]$ are arbitrary, then we obtain the following inequality in the form as

$$
\begin{gathered}
f_{x, y}(\lambda \eta+(1-\lambda) \mu)=f(\lambda[\eta x+(1-\eta) y]+(1-\lambda)[\mu x+(1-\mu) y]) \leq \\
\leq \max \left\{f_{x, y}(\eta), f_{x, y}(\mu), g\left(f_{x, y}(\eta), f_{x, y}(\mu)\right)\right\}
\end{gathered}
$$

for a corresponding function $g: f(D)^{2} \rightarrow \mathbb{R}$ for which $f$ is the general convex function. Thus, the function $f_{x, y}(\lambda)$ is general convex.

Vice versa, let $f_{x, y}(\lambda):[0,1] \rightarrow \mathbb{R}$ be a general convex function over a bisection function $g: f(D)^{2} \rightarrow \mathbb{R}$. Then we obtain that holds the following inequality in the form as

$$
\begin{gathered}
f(\lambda x+(1-\lambda) y)=f_{x, y}(\lambda)=f_{x, y}(\lambda+(1-\lambda) 0) \leq \\
\leq \max \left\{f_{x, y}(1), f_{x, y}(0), g\left(f_{x, y}(1), f_{x, y}(0)\right)\right\}=\max \{f(x), f(y), g(f(x), f(y))\},
\end{gathered}
$$

for all points $x, y \in D$ and for arbitrary $\lambda \in[0,1]$. This means that $f: D \rightarrow \mathbb{R}$ is a general convex function. The proof is complete.

According to problem 24, if $f: J \rightarrow \mathbb{R}$ ( $J$ is an open interval) is a convex function, then for every fixed $x \in J$ the differences quotient $I(x, h)$ is an increasing function of $h$. Consequently it has finite one-sided limits as $h$ tends to zero from the right, and from the left. But these limits are one-sided deviratives of $f$ at $x$ in the following forms:

$$
f_{+}^{\prime}(x):=\lim _{h \rightarrow 0+} I(x, h), \quad f_{-}^{\prime}(x):=\lim _{h \rightarrow 0-} I(x, h) .
$$

10.17. (Differentiation of convex functions). Let $J \subset \mathbb{R}$ be an open interval, and let $f: J \rightarrow \mathbb{R}$ be a convex function. Then at every point $x \in J$ there exist the right derivative $f_{+}^{\prime}(x)$ and the left derivative $f_{-}^{\prime}(x)$, and we have for every $x, y \in J(x<y)$ that is

$$
f_{-}^{\prime}(x) \leq f_{+}^{\prime}(x) \leq f_{-}^{\prime}(y) \leq f_{+}^{\prime}(y) .
$$

Proof. The existence of derivatives $f_{-}^{\prime}(x)$ and $f_{+}^{\prime}(x)$ results from the monotonicity of $I(x, h)$ as a function of $h$, as has been pointed out above, by problem 24. Moreover, for all $x, y, z \in J(x<y<z)$, from problem 23, we obtain

$$
\frac{f(y)-f(x)}{y-x} \leq \frac{f(z)-f(x)}{z-x} \leq \frac{f(z)-f(y)}{z-y} .
$$

Letting that $y \rightarrow x+$ in the first inequality anf that $y \rightarrow z-$ in the second inequality, we obtain (with $f_{-}^{\prime}(x) \leq f_{+}^{\prime}(x)$ ) all inequalities in (66). The proof is complete.

Some remarks. Inequalities (66) show that the functions $f_{+}^{\prime}(x)$ and $f_{-}^{\prime}(x)$ are increasing, and hence at every point $x \in J$ they have one-sided limits. Since $f$ is continuous, the function $I(x, h)$ is continuous with respect to $x$. 
Moreover, we have for all $x, y \in J(x<y)(0<h<y-x)$, the following inequalities

$$
I(x,-h) \leq I(x, h) \leq I(y,-h) \leq I(y, h) ;
$$

only the inequality $I(x, h) \leq I(y,-h)$ requires a motivation, the remaining ones result from the inequality $-h<h$ and from problem 24 . Now,

$$
I(y,-h)=\frac{f(y-h)-f(y)}{-h}=\frac{f((y-h)+h)-f(y-h)}{h} \geq \frac{f(x+h)-f(x)}{h}=I(x, h) .
$$

since $y-h>x$, and the function $I(x, h)$ is monotonic with respect to the first variable, from problem 24. Letting in (67) $h \rightarrow 0+$ we obtain inequalities (66), also. Moreover,

$$
\begin{aligned}
& \lim _{t \rightarrow x+} f_{+}^{\prime}(t)=\lim _{t \rightarrow x+} f_{-}^{\prime}(t)=f_{+}^{\prime}(x), \\
& \lim _{t \rightarrow x-} f_{+}^{\prime}(t)=\lim _{t \rightarrow x-} f_{-}^{\prime}(t)=f_{-}^{\prime}(x) .
\end{aligned}
$$

Geometrically, we notice from the preceding facts and problem 23 that if $f: J \rightarrow \mathbb{R}$ is a convex function but not necessarily differentiable function, then it follows two inequalities: $f(x) \geq f(\xi)+f_{+}^{\prime}(\xi)(x-\xi)$ and $f(x) \geq f(\xi)+f_{-}^{\prime}(\xi)(x-\xi)$ for arbitrary point $\xi \in J$. See: Fig. 11.

Here, the so-called subdifferential is equals the set of all slopes of the straight lines through $(x, f(x))$ which lie beneath the curve determined by $f$ is the stating point for the convex analysis!

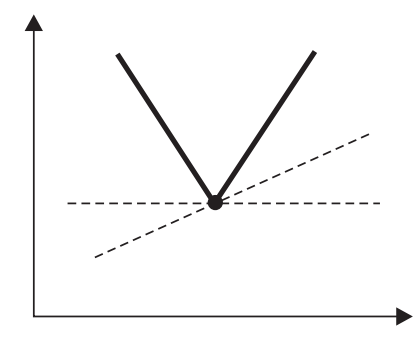

\section{FiguRE 11}

Annotations. We may observe that the convexity of $f$ alone does not imply the monotonicity of $I(x, h)$ with respect to $x$. In this sense, let $g: \mathbb{R} \rightarrow \mathbb{R}$ be a discontinuous additive function, and let $f: \mathbb{R} \rightarrow \mathbb{R}$ be defined by $f(x)=[g(x)]^{2}$ for $x \in \mathbb{R}$. Thus $f$ is J-convex. Fix and $h$ such that $g(h) \neq \overline{0}$. We have $I(x, h)=2 g(x) g(h) / h+[g(h)]^{2} / h$. Hence

$$
g(x)=\frac{h}{2 g(h)}\left(I(x, h)-\frac{1}{h}[g(h)]^{2}\right) ;
$$

i.e., if $I(x, h)$ were increasing with respect to $x$, then the function $g$ would be monotonic, and hence continuous. Consequently $I(x, h)$ cannot be increasing with respect to $x$.

10.18. (Differential conditions of convexity). The convexity of a differentiable function can be inferred from the behaviour of its derivatives as: Let $I \subset \mathbb{R}$ be an open interval, and let $f: I \rightarrow \mathbb{R}$ be a differentiable function. The function $f$ is J-convex if and only if the function $f^{\prime}$ is increasing in $I$. 
Proof. The sufficiently part results from problem 26. Now assume that $f^{\prime}$ is increasing in $I$, and take arbitrary $x, y \in I(x<y)$. We have by the mean-value theorem.

$$
\begin{aligned}
& f(y)-f\left(\frac{x+y}{2}\right)=f^{\prime}(v)\left(y-\frac{x+y}{2}\right)=f^{\prime}(v) \frac{y-x}{2}, \\
& f\left(\frac{x+y}{2}\right)-f(x)=f^{\prime}(u)\left(\frac{x+y}{2}-x\right)=f^{\prime}(u) \frac{y-x}{2},
\end{aligned}
$$

where $u, v \in I$ are points sucht that $x<u<(x+y) / 2<v<y$. Hence $f^{\prime}(u) \leq f^{\prime}(v)$, i.e., this means that the following inequality holds

$$
f\left(\frac{x+y}{2}\right)-f(x) \leq f(y)-f\left(\frac{x+y}{2}\right),
$$

thus, this inequality has been so far established for $x<y$. For $x=y$ this is obvious. The proof is complete.

Annotation. As a direct consequence of this result we have the following fact: Let $J \subset \mathbb{R}$ be an open interval, and let $f: J \rightarrow \mathbb{R}$ be a twice differentiable function. The function $f$ is J-convex if and only if the function $f^{\prime \prime}$ is nonnegative in $J$.

10.19. (General convex functions with two variables, Tas k ovic [2005]). Let $D$ and $C$ be two convex subsets of $\mathbb{R}^{n}$ (or of a real Banach space $E$ ). We say that $f: D \times C \rightarrow \mathbb{R}$ is a general convex function iff there is a function $g: f(D \times C)^{2} \rightarrow \mathbb{R}$ such that

$$
f(\lambda x+(1-\lambda) y, \lambda a+(1-\lambda) b) \leq \max \{f(x, a), f(y, b), g(f(x, a), f(y, b))\}
$$

for all $x, y \in D$, for all $a, b \in C$, and for arbitrary $\lambda \in[0,1]$.

If (Dk) holds for all $x, y \in D$, for all $a, b \in C$, and for some $\lambda \in[0,1]$, then $f$ is a general $\lambda$-convex function.

If $\lambda=1 / 2$, then $f$ is a general $J$-convex function, i.e., if the following inequality holds for some function $g: f(D \times C)^{2} \rightarrow \mathbb{R}$ in the form as

$$
f\left(\frac{x+y}{2}, \frac{a+b}{2}\right) \leq \max \{f(x, a), f(y, b), g(f(x, a), f(y, b))\}
$$

for all $x, y \in D$ and for all $a, b \in C$.

Adequate to the preceding facts, $f: D \times C \rightarrow \mathbb{R}(D$ and $C$ are convex sets $)$ is a general $J$-concave function iff there is a function $d: f(D \times C)^{2} \rightarrow \mathbb{R}$ such that

$$
f\left(\frac{x+y}{2}, \frac{a+b}{2}\right) \geq \min \{f(x, a), f(y, b), d(f(x, a), f(y, b))\}
$$

for all $x, y \in D$ and for all $a, b \in C$.

On the other hand, $f: D \times C \rightarrow \mathbb{R}$ ( $D$ and $C$ are convex sets) is a general concave function iff there is a function $d: f(D \times C)^{2} \rightarrow \mathbb{R}$ such that

$$
\min \{f(x, a), f(y, b), d(f(x, a), f(y, b))\} \leq f(\lambda x+(1-\lambda) y, \lambda a+(1-\lambda) b) .
$$

for all $x, y \in D$, for all $a, b \in C$, and for arbitrary $\lambda \in[0,1]$.

The following statement holds: Let $D \subset \mathbb{R}^{n}$ be an oopen and convex set. If $f$ : $D \times D \rightarrow \mathbb{R}$ is a general J-convex function, then there is a function $g: f(D \times D)^{n} \rightarrow \mathbb{R}$ such that

$f\left(\frac{x_{1}+\cdots+x_{n}}{n}, \frac{a_{1}+\cdots+a_{n}}{n}\right) \leq \max \left\{f\left(x_{1}, a_{1}\right), \ldots, f\left(x_{n}, a_{n}\right), g\left(f\left(x_{1}, a_{1}\right), \ldots, f\left(x_{n}, a_{n}\right)\right)\right\}$

for every $n \in \mathbb{N}$, for all points $x_{1}, \ldots, x_{n} \in D$, and for all points $a_{1}, \ldots, a_{n} \in D$. (The proof of this statement is totally analogous with the proof of Theorem 2.) 
10.20. (General s-convex functions, T a s k o vi ć [2005]). In this part first we will give a definition of an s-convex set. Let $X$ be a linear space over the field $\mathbb{R}$. Any element of the following form as

$$
a^{1 / s} x+b^{1 / s} y \text { for } 0<s \leq 1,
$$

where $a+b=1(a, b \geq 0)$ and $x, y \in X$ will be called an $s$-convex linear combination of $x$ and $y$. A subset $A$ of the space $X$ is said to be $s$-convex set for $0<s \leq 1$, if, whenever it contains $x$ and $y$, it also contains all their $s$-convex linear combinations.

Let $X=\mathbb{R}$, then evidently $A=[0,+\infty)$ is an $s$-convex set for every $0<s \leq 1$. Moreover, if $A \subset X=\mathbb{R}$ is closed and $s$-convex for $0<s \leq 1$, then it is a closed interval containing zero not necessarily as an inner point.

The following characterization of the $s$-convexity holds: $A$ subset $A \subset X$ is $s$-convex if and only if for each $n$ points $x_{1}, \ldots, x_{n} \in A$ and for all $a_{1}, \ldots, a_{n} \geq 0$ satisfying $a_{1}+\cdots+a_{n}=1$ the following condition holds

$$
\left(\sum_{k=1}^{n} a_{k}^{1 / s} x_{k}\right) \in A \quad \text { where } n \geq 2 .
$$

Let the domain of a function $f$ be an $s$-convex subset $A \subset X$ and $f(A) \subset \mathbb{R}$. We call this function $f$ is general $s$-convex for $0<s \leq 1$ if there exists a function $g: F(A)^{2} \rightarrow \mathbb{R}$ such that for all $s$-convex combinations $a^{1 / s} x+b^{1 / s} y$ of $x, y \in A$ the following inequality holds in the form as

$$
f\left(a^{1 / s} x+b^{1 / s} y\right) \leq \max \{f(x), f(y), g(f(x), f(y))\} .
$$

We notice that repalacing $a$ and $b$ in inequality (68) by $a$ and $b$ respectively, we obtain the following inequality of the form as

$$
f(a x+b y) \leq \max \{f(x), f(y), g(f(x), f(y))\},
$$

where $a^{2}+b^{s}=1$ for $a, b \geq 0$. In this sense, the inequalities (68) and (69) are evidently equivalent. The following fact holds.

Proposition 12. Let $A$ be an s-convex set of a linear space $X$ for $0<s \leq 1$. Then the function $f: A \rightarrow \mathbb{R}$ is general s-convex if and only if there is a function $g: f(A)^{n} \rightarrow \mathbb{R}$ such that

$$
f\left(\sum_{k=1}^{n} a_{k}^{1 / s} x_{k}\right) \leq \max \left\{f\left(x_{1}, \ldots, f\left(x_{n}\right), g\left(f\left(x_{1}\right), \ldots, f\left(x_{n}\right)\right)\right\}\right.
$$

for every $n \geq 2$, for all points $x_{1}, \ldots, x_{n} \in A$ and for all real numbers $a_{1}, \ldots, a_{n} \geq 0$ satisfying $a_{1}+\cdots+a_{n}=1$.

In the next let us take nonnegative $b_{1}, \ldots, b_{n}$ such that $b_{1}+\cdots+b_{n}>0$. Then applying Proposition 88 with $a_{k}=b_{k} /\left(b_{1}+\cdots+b_{n}\right)$ we get the following inequality for general $s$-convex functions $f: A \rightarrow \mathbb{R}$ for $0<s \leq 1$ in the form as

$$
f\left(\frac{\sum_{k=1}^{n} b_{k}^{1 / s} x_{k}}{\left(\sum_{k=1}^{n} b_{k}\right)^{1 / s}}\right) \leq \max \left\{f\left(x_{1}\right), \ldots, f\left(x_{n}\right), g\left(f\left(x_{1}\right), \ldots, f\left(x_{n}\right)\right)\right\} .
$$

In the preceding context, inequalities (70) and (71) are equivalent. Also, if the function $f: A \rightarrow \mathbb{R}$ is general $s$-convex, then it is general $\lambda$-convex function for all $0<\lambda \leq s \leq 1$.

On the other hand, in this part we will introduce a definition of a general modular convex function in the following sense. 
Namely, the function $f: A \rightarrow \mathbb{R}$ is called general modular convex if there exist a function $g: f(A)^{2} \rightarrow \mathbb{R}$ and a number $\gamma \geq 1$ such that

$$
f(\alpha x+\beta y) \leq \max \{f(\gamma x), f(\gamma y), g(f(\gamma x), f(\gamma y))\}
$$

for all $x, y \in A$ and for arbitrary numbers $\alpha, \beta \geq 0$ such that $\alpha+\beta=1$.

If $f: A \rightarrow \mathbb{R}$ is a general modular convex function and an upper transversal modular, then in special case we can obtain so-called Musielak-Orlicz $s^{*}$-quasi convex modular $\rho$ in the form as

$$
\rho(\alpha x+\beta y) \leq \alpha^{s *} \rho(\gamma x)+\beta^{s *} \rho(\gamma y)
$$

for all $x, y \in A$, for arbitrary numbers alpha, $\beta \geq 0$ satisfying $\alpha^{s *}+\beta^{s *} \leq 1\left(s^{*}=\right.$ $\min \{1, s\}, 0<s<+\infty)$, and if for some $\gamma \geq 1$ independently of $x, y, \alpha$ and $\beta$.

In connection with this is essential the following class of functions. Namely the function $f: A \rightarrow \mathbb{R}$ is general modular concave if there exist a function $d: f(A) \rightarrow \mathbb{R}$ and a number $\gamma \geq 1$ such that

$$
f(\alpha x+\beta y) \geq \min \{f(\gamma x), f(\gamma y), d(f(\gamma x), f(\gamma y))\}
$$

for all $x, y \in A$ and for arbitrary numbers $\alpha, \beta \geq 0$ satisfying $\alpha+\beta=1$. (For further facts see: Tasković [1993]).

In the contexted of the preceding facts, the function $f: A \rightarrow \mathbb{R}$ is general $s$-concave for $0<s \leq 1$ if there is a function $d: f(A)^{2} \rightarrow \mathbb{R}$ such that for all $s$-convex combinations $a^{1 / s} x+b^{1 / s} y$ of $x, y \in A$ the following inequality holds in the form as

$$
f\left(a^{1 / s} x+b^{1 / s} y\right) \geq \min \{f(x), f(y), d(f(x), f(y))\} .
$$

In the proper preceding manner, replacing $a$ and $b$ in inequality (72) by $a^{s}$ and $b^{s}$ respectively, we obtain the following inequality in the form as

$$
f(a x+b y) \geq \min \{f(x), f(y), d(f(x), f(y))\},
$$

where $a^{s}+b^{s}=1$ for $a, b \geq 0$. In this sense, the inequalities (72) and (73) are evidently equivalent. We give the following illustrations.

Example 1. ( $\varphi$-functions, M a t u s z ews ka [1961]). The function $\varphi: \mathbb{R}_{+}^{0} \rightarrow \mathbb{R}_{+}^{0}$ is called $\varphi$-function if it is nondecreasing, continuous, $f(u)=0$ if and only if $u=0$, and if $\varphi(u) \rightarrow+\infty$ as $u$ to $\infty$. We recall that an $\varphi$-function is $s^{*}$-quasi convex if for $\alpha, \beta \geq 0$ and $\alpha^{s *}+\beta^{s *} \leq 1$ the folloowing inequality holds

$$
\varphi(\alpha u+\beta v) \leq \alpha^{s *} \varphi(\gamma u)+\beta^{s *} \varphi(\gamma v)
$$

for all $u, v \in A$ and for some $\gamma \geq 1$ independently of the points $u, v$ and of the numbers $\alpha, \beta$. In this case $s^{*}=\min \{1, s\}$ for $0<s<+\infty$.

Example 2. (O r l i c z [1932], $s^{*}$-quasi convex modular). Let $(\Omega, \Sigma, \mu)$ be a measure space and let $X$ be a set of all $\Sigma$-measurable functions on $\Omega$. If $\varphi$-function is an $s^{*}$-quasi convex, then Orlicz modular of the form as

$$
\rho(x)=\int_{\Omega} \varphi(|x(t)|) d_{\mu}(t)
$$

is an $s^{*}$-quasi convex function and the Orlicz space $L^{\varphi}=X_{\rho}$ is an $s^{*}$-quasi normed space with the $s^{*}$-quasi norm of the form as

$$
|x|_{\rho}=\inf \left\{u>0: \rho\left(\frac{x}{u^{1 / s *}}\right) \leq 1\right\}
$$

in the space $X_{\rho}=\{x \in X: \rho(\lambda x) \rightarrow 0$ as $\lambda \rightarrow 0+\}$. What else, $L^{\varphi}$ is a quasi normed space with the consequant norm on quasi normed spaces $X$ and $Y$. 
10.21. (The slasses $d(\varphi)$-functions and $d\left(L^{\varphi}\right)$-spaces, T a s k o v i ć [2000]). In this part, by a $d(\psi)$-function I shall understand a continuous nonincreasing function $\psi: \mathbb{R}_{+}^{0} \rightarrow \mathbb{R}_{+}^{0}:=[0,+\infty)$ for which $\psi(u) \rightarrow 0$ as $u \rightarrow+\infty$ and $\psi(0)=b$ (for some $(0<b \leq+\infty)$.

In this sense, for the case $b=+\infty$, I shall understand that $\psi(u) \rightarrow+\infty$ as $u \rightarrow 0$, where $f \mid \mathbb{R}_{+}=(0,+\infty)$. On the Figs. $12,13,14$ and 15 we have the essential forms of $d(\psi)$-functions.

Classe $d(\psi)$-functions appear often in various problems of nonlinear analysis and have a certain analogy (although essential unlike) with the class of $\varphi$-functions from $\mathrm{M} \mathrm{a}$ t u s z e w ska [1961].

In our context, first time, the forms of $d(\psi)$-functions are appear in the connection with the transversal lower (modular) spaces in 1998 from: T a s k o vi ć [2000] and [2005].

The following conditions appear often in various problems in which the $d(\psi)$-functions are of importance:

$$
\lim _{u \rightarrow 0} u \psi(u)=0 \text { and } \lim _{u \rightarrow \infty} u \psi(u)=0,
$$

in the contrast with appears of the $\varphi$-functions in various problems in the forms:

$$
\lim _{u \rightarrow 0} \frac{\varphi(u)}{u}=0 \quad \text { and } \quad \lim _{u \rightarrow \infty} \frac{\varphi(u)}{u}=+\infty .
$$

Let $\Omega$ be a nonempty set and let $\Sigma$ be a sigma-algebra of subsets of $\Omega$. Also, let mu be a nonnegative, nontrivial, complete, sigma-finite measure on $\Sigma$. We take as $X$ the space of all extended real valued, $\Sigma$-measurable functions on $\Omega$ with equality mu-almost everywhere. Let $\psi$ be a $d(\psi)$-function, then

$$
\rho(x)=\int_{\Omega} \psi(x(t)) d \mu
$$

is a lower transversal modular in $X$. Moreover, if $\psi$ is concave, then $\rho$ is concave.

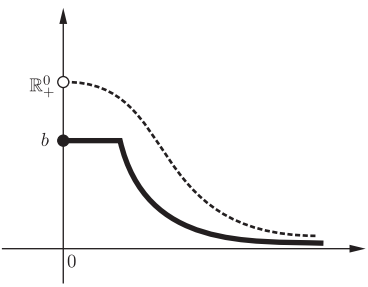

Figure 12

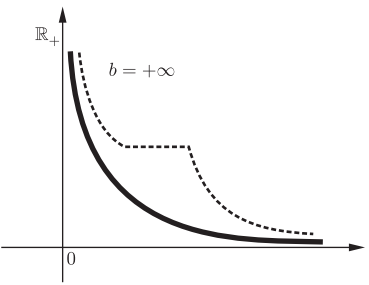

Figure 13

The set of all $x \in X$ for which $\rho(x)+\infty$ is called the $d(\psi)$-class, till the lower transversal modular space $d\left(X_{\rho}\right)$ denoted by $d\left(L^{\psi},(\Omega, \Sigma, \mu)\right)$ or $d\left(L^{\psi}\right)$. It is evident that $d\left(L^{\psi}\right)$ is the smallest linear space containing the $d(\psi)$-class.

An inequality. If $f: \mathbb{R}_{+}^{0} \rightarrow \mathbb{R}_{+}^{0}$ is a continuous and strictly decreasing function with property $f(0)=b \in \mathbb{R}_{+}$, then for $a>0$ the following inequality holds in the form

$$
\left(a-f^{-1}(0)\right) b \leq \int_{0}^{a} f(x) d x-\int_{0}^{b} f^{-1}(x) d x,
$$

where the equality holds in (74) if and only if $f(a)=0$. (In (74) $f^{-1}$ is the inverse function of $f$ ).

It is easily seen that the function $f: \mathbb{R}_{+}^{0} \rightarrow \mathbb{R}_{+}^{0}$ from inequality (74) is a function in the classe of $d(\psi)$-functions i.e., $f \in d(\psi)$. 


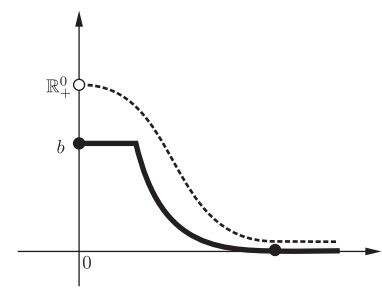

FIGURE 14

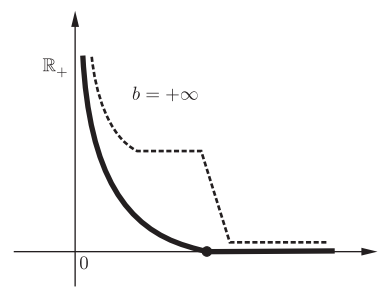

FIGURE 15

On the other hand, from inequality (74), we can defined two complementary functions in the following sense. We set

$$
\psi(u)=\int_{0}^{u} f(t) d t, \quad \text { and } \quad \psi^{*}(u)=\int_{0}^{u} f^{-1}(t) d t,
$$

for $u \geq 0$. Then, the functions $\psi(u)$ and $\psi^{*}(u)$ are complementary in the sense of inequality (74). It is easily proved that they satisfy the following inequality of the form

$$
u v-v f^{-1}(0) l \psi(u)-\psi^{*}(v)
$$

for all $u, v \geq 0$; and that

$$
\psi^{*}(u)=\inf _{v \geq 0}\left(\psi(v)-u v+v f^{-1}(0)\right)
$$

and

$$
\psi(u)=\sup _{v \geq 0}\left(u v-v f^{-1}(0)+\psi^{*}(v)\right),
$$

for every $u \geq 0$, where the infimum being reached at $v=f^{-1}(u)$ in the first case, ant the supremum at $v=f(u)$ in the second case. Now, it is easily proved that

$$
\|x\|_{\psi}^{*}=\sup \left\{\int_{\Omega} x(t) y(t) d \mu: y \in X, \int_{\Omega} \psi^{*}(y(t)) d \mu \leq 1\right\},
$$

is a lower transversal norm in $d\left(L^{\psi}\right)$, and, on the other hand, that is

$$
\|x\|_{\psi}=\inf \left\{u>0: \int_{\Omega} \psi\left(\frac{x(t)}{u}\right) d \mu \leq 1\right\} ;
$$

also, is a lower transversal norm in $d\left(L^{\psi}\right)$. Obviously is heare similarity with the Luxemburg and Orlicz norms.

Further on $d\left(L^{\psi}\right)$-spaces. An extension of the $d\left(L^{\psi}\right)$-space is the following. We take a function $\psi: \mathbb{R}_{+}^{0} \times \Omega \rightarrow \mathbb{R}_{+}^{0}$ such that: $\psi(u, t)=b$ (for some $0<b \leq+\infty$ ) if and only if $u=0, \psi(u, t)$ is a continuous and nonincreasing function of $u \geq 0$ for a.e. $t \in \Omega, \psi(u, t)$ is $\Sigma$-measurable in $\Omega$ for every $u \geq 0$ and $\psi(u, t) \rightarrow \infty$ as $u \rightarrow \infty$ for a.e. $t \in \Omega$. Then

$$
\rho(x)=\int_{\Omega} \psi(x(t), t) d \mu
$$

is a lower transversal modular in $X$. On the other hand, one may consider alos $d\left(L^{\psi}\right)$ spaces of vector valued functions with falues in a lower transversal normed space $(E,\|\cdot\|)$ which is lower complete. The lower transversal modular $\rho$ may be written then in the form

$$
\rho(x)=\int_{\Omega} \psi(\|x(t)\|, t) d \mu
$$


with suitable measurability for the functions $x$. Also, this may be still extended to the form

$$
\rho(x)=\int_{\Omega} \psi(x(t), t) d \mu,
$$

where $\psi$ becomes a map of $E \times \Omega$ in $\mathbb{R}_{+}^{0}$. For further facts of this see: Ta s k o v ić [2005].

Proof of inequality (74). From the Figures 16 and 17 we have a geometric proof of (74). Meanwhile, for an analytic proof of inequalityu (74), we set

$$
g(a)=a b-b f^{-1}(0)-\int_{0}^{a} f(x) d x,
$$

and consider $b>0$ as a parametar. Since $g^{\prime}(a)=b-f(a)+f(0)$, and $f$ is strictly decreasing, we have

$$
\begin{gathered}
g^{\prime}(a)>0 \text { for } \quad 0<a<f^{-1}(b-f(0)), \\
g^{\prime}(a)=0 \quad \text { for } \quad a=f^{-1}(b-f(0)), g^{\prime}(a)<0 \\
\text { for } \quad a>f^{-1}(b-f(0))
\end{gathered}
$$

and thus, $g(a)$ is a maximum of $g$ for $a=f^{-1}(b-f(0))$. Therefore, integrating by parts, we obtain

$$
g\left(f^{-1}(b-f(0))\right)=f(0) f^{-1}(b-f(0))-b f^{-1}(0)+\int_{f(0)}^{b-f(0)} f^{-1}(y) d y,
$$

i.e., for $b=f(0)$ we have $g(a) \leq g\left(f^{-1}(0)\right)$, and thus calculating we get (74). The proof is complete.

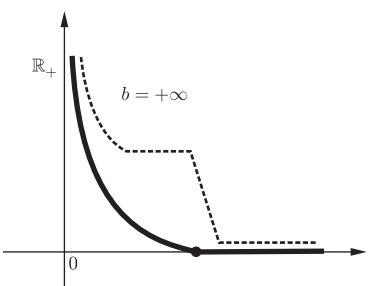

FigURE 16

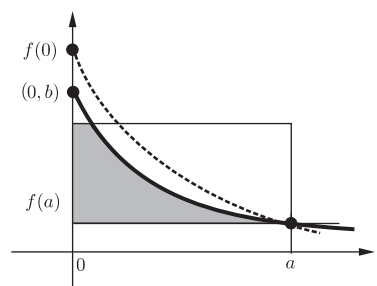

FiguRE 17

Further annotations. We notice that Figs. 5 and 6 affirm that inequality (74) is justified. On the other hand, if we consider the areas by Figs. 5 and 6 , then directly for a strictly decreasing function $\mathrm{f}$ in $d(\psi)$ we obtain the following inequality of form

$$
a f(a) \leq \int_{0}^{a} f(x) d x-\int_{f(a)}^{b} f^{-1}(x) d x,
$$

where $f(0) \geq b$ and $a>0$. Equality holds in (75) if and only if $f(0)=b$ and $f(a)=0$.

We notice that inequality (75) is ties with some inequalities in: B o a s-M a r c u s [1974], M i tr in ovi ć-P eč a r i ć-F in k [1993] and Tas ković [2001].

Class of $d(\psi)$-functions. We shall say that a continuous and nonincreasing ${ }^{5}$ function $\psi: \mathbb{R}_{+}^{0} \rightarrow \mathbb{R}_{+}^{0}$ in the classe $d(\psi)$ or that is a $d(\psi)$-function if

$$
\psi(\infty)=\lim _{n \rightarrow \infty} \psi(u)=0
$$

\footnotetext{
${ }^{5}$ Decreasing functions. For the decreasing function $\psi: \mathbb{R}_{+}^{0} \rightarrow \mathbb{R}_{+}^{0}$ holds the following properties: for every $s \geq 0$ there exist the following expression (we set on convention
} 
and $\psi(0)=b(0<b \leq+\infty)$; where in the case $b=+\infty$ we understand a function of the form that $f: \mathbb{R}_{+}^{0} \cup\{+\infty\}$.

Namely, in the case $b=+\infty$, we can $\psi$, comprehend and as a restriction on the set $\mathbb{R}_{+}$, i.e., $\psi \mid \mathbb{R}_{+} \rightarrow \mathbb{R}_{+}^{0}$. The elements of the class $d(\psi)$ we denoted by $\psi, \xi, \varphi, \ldots$ and $\xi \in d(\psi)$ is a measurable function. Also, $\xi(u)=\xi \circ u \mathrm{u}$ is a measurable function for the arbitrary measurable function $u: \mathbb{R}_{+} \rightarrow \mathbb{R}_{+}^{0}$

For the function $\psi \in d(\psi)$ we defined on the right inverse function $\psi^{*}: \mathbb{R}_{+}^{0} \rightarrow \mathbb{R}_{+}^{0}$ in the folowing sense

$$
\psi^{*}(t)=\sup \psi^{-1}(t, b)=\inf \psi^{1}([0, t]),
$$

where $0<b \leq+\infty$. It is easily proved that $\psi^{*} \in d(\psi)$ and $\psi^{* *}=\psi$, i.e., $\psi^{*}$ is an idempotent mapping. Precisely, this means that $\psi$ and $\psi^{*}$ are reciprocally on the right inverse functions.

Proposition 13. Let $\psi \in d(\psi)$. Then the following characteristic facts hold:

(a) $\psi^{*}(\psi(s)) \leq s$ for every $s \geq 0$.

(b) $t>\psi(s)$ simplies that is $\psi^{*}(t)<s$.

(c) $\varphi(s)=a \psi(b s)$ implies $\varphi^{*}(t)=\frac{1}{b} \psi^{*}\left(\frac{t}{a}\right)$ for $a, b>0$.

(d) $\psi^{*}(\psi(s)-\varepsilon) \geq s$ for everu $s>0$ and $0<\varepsilon<\psi(s)$.

A brief proof of this statement we can to make in the proper manner from book of M usielak [1976]. Also see: M a t uszewska [1961] and Tasković [2005].

Annotation. If $\psi \in d(\psi)$ with the values $t$, then $\psi^{*}(t)=\inf \psi^{-1}(\{t\})$. Otherwise, if $\psi^{*}$ (for $\psi \in d(\psi))$ is a continuous function in the point $\psi(s)$, then, from Proposition 1, the following equality holds: $\psi^{*}(\psi(s))=s$.

The classe of $d(M)$-functions. In the further, a function $G: \mathbb{R}_{\rightarrow} \mathbb{R}_{+}^{0}$ is called an $M$-function if there is a function $\psi \in d(\psi)$ such that

$$
G(s)=\int_{0}^{|s|} \psi(t) d t .
$$

By $d(M)$ we shall denote the set of all $M$-functions. If (76) holds, then we say that the function $G$ determined by the function $\psi$.

$$
\begin{gathered}
\psi_{-}(s)=\psi(s-0)=\lim _{h \rightarrow 0+} \psi(s-h)=\inf ([0, s])= \\
\inf \psi((t, s)) \text { for } 0 \leq t<s \text { and } \\
\psi(s+0)=\lim _{h \rightarrow 0+} \psi(s+h)=\sup \psi((s, \infty))= \\
\sup \psi((s, t)) \text { for } s<t ;
\end{gathered}
$$

where holds the following inequalities in the forms: $\psi_{+} \leq \psi \leq \psi_{-}$and $\psi_{-}(t) \leq \psi_{+}(s)$ for $s<t$.

On the other hand, a decreasing function $\psi: \mathbb{R}_{+}^{0} \rightarrow \mathbb{R}_{+}^{0}$ is from the right continuous if holds the following equality

$$
\psi(\sup S)=\inf \psi(S)
$$

for every nonempty bounded set $S \subset \mathbb{R}_{+}^{0}$. In this sense, if holds and the following equality in the form (continuity from the left)

$$
\psi(\inf S)=\sup \psi(S),
$$

then we say, that the mapping, $\psi$ is continuous. For further facts on the decreasing mappings see: Taskovi ć [2005]. 
The value of the $M$-function, itself is the magnitude of the area of the corresponding curvilinear trapezoid. If follows from representation (76) that every M-function is even, thut for $s \in \mathbb{R}_{+}^{0}$ and $G \in d(M)$ directly we have the following inequalities

$$
\operatorname{as\psi }\left(\frac{s}{a}\right)<G(s)<s \psi(0),
$$

i.e., in an equivalent form,

$$
a^{2} s \psi(s)<G(a s)<a s \psi(0)
$$

for $s>0$ and for $0<a \leq 1$. Hence, from the preceding inequalities (77) and (78), directly calculating, we obtain the following inequalities in the form

$$
\psi_{-}(\max \{s, t\}) \leq \frac{G(s)-G(t)}{s-t} \leq \psi_{+}(\min \{s, t\}) ;
$$

and thus, from the preceding facts, we have

$$
G(s)=\max _{t \geq 0}\left\{g(t)+(s-t) \psi_{=}(s)\right\}=\min _{t \geq 0}\left\{G(t)+(s-t) \psi_{+}(s)\right\} .
$$

Proposition 14. The function $f: \mathbb{R} \rightarrow \mathbb{R}$ belongs to the classe $d(M)$ if and only if the following facts hold: $f(x)=0$ if and only if $s=0, f \in B C\left(\mathbb{R}_{+}^{0}\right)^{6} f$ is continuous even, and

$$
0 \leq \frac{f(\infty)}{\infty} \leq b=\frac{f(0)}{0} \quad(0<b \leq+\infty)
$$

A brief proof of this statement may be found in: Tas k o vi ć [2005]. As an immediate application of this result and the preceding facts we have the following inequalities:

$$
\begin{gathered}
G(a s) \geq a G(s) \text { for } s \geq 0 \text { and } 0 \leq a \leq 1 . \\
G(a s) \leq a G(s) \text { for } s \geq 0 \text { and } a \geq 1 . \\
\quad \frac{G(t)}{t}<\frac{G(s)}{s} \text { for } 0 \leq s<t . \\
G(s+t)<G(s)+G(t) \text { for } s, t>0 .
\end{gathered}
$$

We notice that properties of the function $G$ directly to bring about properties hers the inverse function defined by $G^{-1}=\left(G \mid \mathbb{R}_{+}^{0}\right)^{-1}$.

The function $G^{-1}$, defined in the preceding sense, is continuous, difference is two convex functions, i.e., $G^{-1} \in B C\left(\mathbb{R}_{+}^{0}\right)$, and strictly decreasing on $\mathbb{R}_{+}^{0}$.

If to make corresponding replacements variables, from inequalities (80)-(83), for the function $G^{-1}$ we obtain the following inequalities:

$$
\begin{gathered}
G^{-1}(a s) \leq a G^{-1}(s) \text { for } s \geq 0 \text { and } 0 \leq a \leq 1 . \\
G^{-1}(a s) \geq a G^{1}(s) \text { for } s \geq 0 \text { and } a \geq 1 . \\
\frac{t}{G^{-1}(t)}<\frac{S}{G^{-1}(s)} \text { for } 0 \leq s<t . \\
G^{-1}(s+t)>G^{-1}(s)+G^{-1}(t) \text { for } s, t>0 .
\end{gathered}
$$

We notice that equality holds in (80), (81), (84) and (85) if and only if the point $(s, a)$ is on edge region in which the preceding inequalities hold.

Complementary $M$-function. For a $M$-function hers the complementary function (in the sense of inequalities (77)) is the function $G^{*}$ in the following form:

$$
G^{*}(t)=\int_{)}^{|t|} \psi *(s) d s .
$$

\footnotetext{
${ }^{6} B C\left(\mathbb{R}_{+}^{0}\right)$ denoted the set of all functions which are difference of two convex functions on $\mathbb{R}_{+}^{0}$.
} 
From the some former facts, complementary mapping is an idempotent mapping of the classe $d(M)$ into itself. From Proposition 13 we have that

$$
A(s)=a G(b s) \text { implies } A^{*}(s)=a G^{*}\left(\frac{t}{a b}\right),
$$

where $a, b>0$ are constants.

As an immediate consequence of the former inequality (74) is the following statement.

Theorem 59. Let the function $G \in d(M)$. Then for mutually complementary functions $G$ and $G^{*}$ the following inequality holds in the form

$$
\left(s-\psi^{-1}(0)\right) t \leq G(s)-G^{*}(t)
$$

for $s, t \geq 0$, where equality holds in this case if and only if $\psi(s)=0$.

From the preceding Theorem 59 as an immediate consequenca we have the quasilinear representation of an arbitrary complementary function in the form

$$
G^{*}(t)=\min _{s \geq 0}\left(G(s)-\left[s-\psi^{-1}(0)\right] t\right) .
$$

The comparison of $\boldsymbol{M}$-functions. In the sequel, an essential role will be played by the rapidity of growth of the values of an $M$-function as $n \rightarrow \infty$. In connection with this, for the function $G \in d(M)$ we shall say that before the function $R \in d(M)$, in note $G \prec R$ or $G(s) \prec R(s)$, if there exists constant $K>0$ such that

$$
G^{-1}(s) \leq R^{-1}(k s) \text { for enough large } s .
$$

Otherwise, we shall say that the $M$-functions $G$ and $R$ are equivalent in write $G \sim R$ or $G(s) \sim R(s)$ if $G \prec R$ and $R \prec G$, i.e., if there exist constants $K, k>0$ such that

$$
R^{-1}(k s) \leq G^{-1}(s) \leq R^{1-}(K s) \text { for enough large } s .
$$

We notice that the binar relation $\prec$ defined a quasiorder in $d(M)$, which means that $\sim$ is an equivalente relation in $d(M)$ agreed with $\sim$; but the relation $\leq$ defined is on the quotient set $d(M) / \sim=\{/ G /: G \in d(M)\}$ with

$$
[G] \leq[R] \quad \text { if and only if } G \prec R,
$$

and she is a partial order relation on $d(M) / \sim$. In connection with this, from (80) and (81), directly calculating we obtain

$$
G^{-1}(s \min \{a, 1\}) \leq a G^{-1}(s) \leq G^{-1}(s \max \{a, 1\})
$$

for $a>1$ and for $s \geq 0$; hence, from the preceding facts, we have $G \sim a G$. Since $G(s) \sim G(b s)$ for $b>0$, thus we obtain

$$
G(s) \sim a G(b s) \text { for all } a, b>0 .
$$

Some annotations. We notice that in (89) we can suppose that there exists constant $K \geq 1$ instead $K>0$. Indeed, if holds (89) for $s \geq z$ and if $r<z$, then for $r \leq s \leq z$ the following inequality holds

$$
G^{-1}(s) \leq R^{-1}\left(K_{0} s\right), \quad \text { for } \quad K_{0}=\max \left\{1, \sup _{r \leq s \leq z}\left(\frac{G^{-1}(s)}{R^{-1}(s)}\right)\right\} ;
$$

and thus, for $K_{1}=\max \left\{K, K_{0}\right\}$, we obtain that is $G^{-1}(s) \leq R^{-1}\left(K_{1} s\right)$ for $s \geq r$.

From this, for an arbitrary constant $r>0$, we have that fact $G \prec R$ is an equivalent with the fact: that there exists constant $K \geq 1$ such that

$$
G^{-1}(s) \leq R^{-1}(K s) \quad \text { for every } s \geq r .
$$


In connection with this, also we notice that the fact $G \prec R$ is equivalent with the fact that there exist constants $K_{0}, K>0$ such that

$$
G^{-1}(s) \leq K_{0}+R^{-1}(K s) \text { for every } s \geq 0 .
$$

Indeed, let $G^{-1}(s) \leq R^{-1}(K s)$ for every $s \geq 0$. Thus we have $G^{-1}(s) \leq K_{0}+R^{-1}(K s)$ for some $K_{0}>0$, i.e., holds (19). Reversed, if holds (91) and if $K_{0}=R^{-1}\left(K_{1}\right)$ for $K_{1}>0$, from (87) we have

$$
\begin{gathered}
G^{-1}(s) \leq R^{-1}\left(K_{1}\right)+R^{-1}(K s) \leq R^{-1}\left(K_{1} s\right)+R^{-1}(K s) \leq \\
R^{-1}\left(K_{1} s+K s\right)=R^{-1}\left(\left(K_{1}+K\right) s\right)
\end{gathered}
$$

for $s \geq 1$. This means that $G \prec R$ is equivalent with inequality (91).

On the other hand, from properties of $M$-functions and the order relation $\prec$, directly follow that $G \prec R$ is equivalent with the fact:

$$
\inf _{b \geq 1}\left(\lim _{s \rightarrow \infty} \sup \frac{G^{-1}(s)}{R^{-1}(b s)}\right)=0 .
$$

In connection with the preceding partial ordering, let $G \prec R$ for $G, R \in d(M)$ have the following means: that there exists constant $K>0$ such that

$$
G^{-1}(s) \leq K R^{-1}(s) \text { for enough large } s ;
$$

and let $G \backsim \sim$ for $G, R \in D(M)$ have the following means $G \prec R \in d(M)$ have the following means $G \prec R$ and $R \prec G$, i.e., this means that there exist two constants $K$, $k>0$ such that

$$
k R^{-1}(s) \leq G^{-1}(s) \leq K R^{-1}(s) \text { for enough large } s .
$$

It is easily seen that $\prec$ is a quasiorder, till $\sim$ is an equivalence relation in the $d(M)$ class. The fact $G \prec R$ is equivalent with the fact: that there exist two constants $K_{0}$, $K>0$ such that

$$
G^{-1}(s) \leq K_{0}+K R^{-1}(s) \text { for every } s \geq 0 .
$$

The $d\left(\Delta_{2}\right)$-condition. The following condition in order that an $d(M)$-class be linear (i.e., identical with the $d\left(L^{\psi}\right)$-space) is essential.

We say that the M-function $G$ satisfies the $d\left(\Delta_{2}\right)$-condition for large values of $\mathrm{s}$ if there exists constant $K>0$ such that

$$
G^{-1}(2 s) \leq K G^{-1}(s) \text { for enough large } s .
$$

If $M$-function $G$ satisfies (92) for every $s \geq 0$, then we say that $G$ satisfies $d\left(\Delta_{2}\right)$-condition for every $s \geq 0$. The class of all $M$-functions which satisfies $d\left(\Delta_{2}\right)$-condition denoted by $d\left(M_{2}\right)$. In this context, the mappings $p_{\infty}, q_{\infty}: d(M) \rightarrow[0,1]$ we define by:

$$
\begin{aligned}
p_{\infty}(G) & =\liminf _{s \rightarrow \infty} \frac{s \psi(s)}{G(s)} \text { and } \\
q_{\infty} & =\limsup _{s \rightarrow \infty} s \frac{\psi(s)}{G(s)} ;
\end{aligned}
$$

for which we have $p_{\infty}(b G(a s))=p_{\infty}(G)$ and $q_{\infty}(b G(a s))=q_{\infty}(G)$ for arbitrary parametars $a, b>0$. Since $s \mapsto s^{-r} G(s)$ for $s \geq t$ is a nonincreasing function if and only if

$$
\frac{s \psi(s)}{G(s)} \leq r \quad \text { for every } \quad s \geq t,
$$

and since $s \mapsto s^{-1} G(s)$ for $s \geq t$ is a nondecreasing function only if

$$
\frac{s \psi(s)}{G(s)} \geq r \quad \text { for every } \quad s \geq t
$$


thus, we obtain directly the following equalities for functions $p_{\infty}(G)$ and $q_{\infty}(G)$ in the following forms:

$$
p_{\infty}(G)=\sup \left\{\operatorname{rin}(0,1): s^{-1}(G(s) \quad \text { increasing for } \quad s \geq t\}\right.
$$

and

$$
q_{\infty}(G)=\inf \left\{r \in(0,1): s^{-r} G(s) \quad \text { decreasing for } \quad s \geq t\right\} .
$$

Otherwise, from the preceding facts and geometric reasons we can considered the mappings $\mathcal{L}_{\infty}(G), \mathcal{Z}_{\infty}(G): d(M) \rightarrow[1, \infty)$ defined by

$$
\begin{gathered}
\mathcal{L}_{\infty}=\liminf _{s \rightarrow \infty} \frac{s \psi(0)}{G(s)}, \text { and } \\
\mathcal{Z}_{\infty}=\limsup _{s \rightarrow \infty} \frac{s \psi(0)}{G(s)}
\end{gathered}
$$

for which, also, $\mathcal{L}_{\infty}(b G(a s))=\mathcal{L}(G)$ and $\mathcal{Z}_{\infty}(b G(a s))=\mathcal{Z}_{\infty}(G)$ for arbitrary parameters $a, b>0$. Also, the mappings $p, q: d(M) \rightarrow[0,1]$ we define by:

$$
\begin{gathered}
p(G)=\inf _{s>0} \frac{s \psi(s)}{G(s)}, \quad \text { and } \\
q(G)=\sup _{s>0} \frac{s \psi(s)}{G(s)}
\end{gathered}
$$

and thus holds: $1 \leq \mathcal{L}(G) \leq \mathcal{L}_{\infty}(G) \leq \mathcal{Z}_{\infty}(G) \leq \mathcal{Z}(G)$. Also, for the preceding mapping shold and many other properties, see: Task ovi ć [2005].

Annotations. From the preceding considers we see that for further work can be essential the following condition: there exists a constant $K>0$ such that

$$
G\left(\frac{s}{2}\right) \leq K G(s) \quad \text { for enough large } s
$$

Also, from the preceding considers and the properties of the $p$ and $q$ functions, for an arbitrary function $G \in d(M)$ holds the following inequalities:

$$
\begin{aligned}
\frac{1}{q} s \psi(s) & \leq G(s) \leq \frac{1}{p} s \psi(s), \\
\min \left\{a^{p}, a^{q}\right\} G(s) & \leq G(a s) \leq \max \left\{a^{p}, a^{q}\right\} G(s), \\
G\left(\min \left\{a^{1 / p}, a^{1 / q}\right\} s\right) & \leq a G(s) \leq G\left(\max \left\{a^{1 / p}, a^{1 / q}\right\} s\right) \\
\frac{p}{q} \min \left\{a^{p-1}, a^{q-1}\right\} & \leq \frac{\psi(a s)}{\psi(s)} \leq \frac{q}{p} \max \left\{a^{p-1}, a^{q-1}\right\}
\end{aligned}
$$

The functions $\rho_{G}$. Let $S$ be a closed interval on real line and $\mathcal{M}=\mathcal{M}(S, \mu)$ is a set of all extension real mu-measurable, functions on $S$. For an $M$-function $G$ we define on the quotient set $A=\mathcal{M} / \equiv$ the function $\rho_{G}: A \rightarrow \mathbb{R}^{*}:=\mathbb{R} \cup\{ \pm \infty\}$ with

$$
\rho_{G}(x)=\int_{S} G(x(s)) d s,
$$

at to what $\rho_{G^{*}}\left(\right.$ or $\rho_{G}^{*}$ ) suitable the complementary function. This definition is correct because

$$
x \equiv y \quad \text { implies } \quad \rho_{G}(x)=\rho_{G}(y)
$$

and, from the fact that $G$ is an even function arise that $\rho_{G}$ is even, i.e., $\rho_{G}(|x|)=\rho(x)$. Also, $\rho_{G}(u)=0$ if and only if $\mathrm{u}$ equiv 0 , and

$$
\inf \{u, v\} \equiv 0 \quad \text { implies } \quad \rho_{G}(u+v)=\rho_{G}(u)+\rho_{G}(v)
$$

and, $u \leq v$ implies $\rho_{G}(u) \leq \rho_{G}(v)$, and

$$
\rho_{G}(a u+b v) \leq a \rho_{G}(u)(u)+b \rho_{G}(v) \quad \text { for } \quad a, b \geq 1 ;
$$


at tho what for the function $G \in d(M)$ holds the following inequality

$$
\left[u\left(v-\psi^{-1}(0)\right)\right] d s \leq \rho_{G}(u)-\rho_{G}^{*}(u) .
$$

It follows from this inequality and from Levi's theorem that holds quasilinear representation in the following form:

$$
\rho_{G}(u)=\sup _{\rho_{G}^{*}(v)<\infty}\left(\int\left[u\left(v-\psi^{-1}(0)\right)\right] d s+\rho_{G}^{*}(v)\right) .
$$

We set $H=\{s \in S: u(s) \geq v(s)\}$ and $H_{1}=S H$. Then we have $\sup \{u, v\}=$ $u \chi_{H}+v \chi_{H_{1}}$, and thus from (98) we obtain $\rho_{G}(\sup \{u, v\}) \leq \rho_{G}(u)+\rho_{G}(v)$, i.e., by induction, for $n \in N$, the following inequality holds:

$$
\rho_{G}\left(\sup \left\{u_{1}, \ldots, u_{n}\right\}\right) \leq \rho_{G}\left(u_{1}\right)+\cdots+\rho_{G}\left(u_{n}\right) .
$$

We notice that some properties of the $M$-functions have influence and on the some properties of $\rho_{G}$ functions. In this sense hold the following facts:

(a) The fact $G \prec R$ is equivalent with the fact: that there exist constants $K_{0}, K>0$, such that holds the following inequality

$$
\rho_{G^{-1}}(u) \leq K_{0}+K \rho_{R^{-1}}(u) \text { for every } u \in A^{+} *,
$$

where $A^{+}$is positive order cine with the ordering $\leq$in $A$, i.e, $A^{+}=\{|u| \in A:[u] \geq[0]\}$.

(b) The fact $G \prec R$ is equivalent with the fact: that there exist constants $K_{0}, K>0$ such that holds the following inequality

$$
\rho_{G^{-1}}(u) \leq K_{0}+\rho_{R^{-1}}(K u) \text { for every } u \in A^{+} .
$$

Proof. Let $G \prec R$. If in inequality (91) put $u(s)$ in the place $s$ and afterwards take the intergral over $S$, directly we obtain

$$
\rho_{G^{-1}}(u) \leq K_{0} \mu(S)+\rho_{R^{-1}}(K u),
$$

i.e., (99) holds. Reversed, to serve for the formula $\rho_{G^{-1}}\left(b \chi_{H}\right)=G^{-1}(b) \mu(H)$ for $b \in H$ and $H \subset S$, from (99) for $u=u_{t}=t \chi_{S}(t \geq 0)$, we obtain

$$
G^{-1}(t) \leq \frac{K_{0}}{\mu(S)}+R^{-1}(K t)
$$

whence, again from inequality (91), arise the fact $G \prec R$. The proof is complete.

Boundness of set on $\rho_{G}$. The set $X \subset A$ is called the $G$-bounded iff $\sup \rho_{G}(C)<$ $+\infty$. In this sense holds the following statement.

Theorem 60. Let holds the preceding designate. Then the following are mutually equivalent facts:
(a) $G \prec R$.
(b) $\rho_{R}^{-1}\left(\mathbb{R}_{+}^{0}\right) \subset \rho_{G}^{-1}\left(\mathbb{R}_{+}^{0}\right)$.
(c) $\sup \rho_{G}\left(\left(\rho_{G}^{-1}([0, a])\right)<+\infty\right.$ for some $a>0$.
(d) Every $R$-bounded set is $G$-bounded.

For the proof of this statement we utilize Levi's theorem and some properties of $G$ and $\rho_{G}$ functions.

We set $d\left(L_{G}^{*}(S)\right)=d\left(L_{G}^{*}\right)=\left\{x \in A: \rho_{G}(x)<+\infty\right\}$. If utilize only linearity of the space $L$, we can further consider structure of $d\left(L_{G}^{*}\right)$ as a subset of linear space $L$. In this sense, we set

$$
d\left(L_{G}(S)\right)=d\left(L_{G}\right)=\left\{x \in A: a x \in d\left(L_{G}^{*}\right) \text { for some } a>0\right\},
$$

and $d\left(L_{G}^{+}\right)=\left\{u \in d\left(L_{G}\right): u \geq 0\right\}$. For the linear space $d\left(L_{G}\right)$ we say that determinate with the $M$-function $G$. Then hold the following facts: $d\left(L_{G}^{*}\right) \subset L=L_{1}(S), d\left(L_{G}\right) \subset L$, and: 
(a) $d\left(L_{R}^{*}\right) \subset d\left(L_{G}^{*}\right)$ if and only if $G \prec R$.

(b) $d\left(L_{F}\right) \subset d\left(L_{G}\right)$ if and only if $G \prec R$.

From this facts arise that the inequalities hold $d\left(L_{G}^{*}\right) \subset d\left(L_{R}^{*}\right)$ and $d\left(L_{G}\right) \subset d\left(L_{R}\right)$ if and only if $G \sim R$ and $G \sim R$, respectively.

This facts exhibit that all functions of one sim - class determined only one linear space $d\left(L_{G}\right)$, where the mapping of $[G]$ and $d\left(L_{G}\right)$ is bijective. If in the set

$$
\mathcal{D}=\left\{d\left(L_{G}\right): G \in d(M)\right\}
$$

defined the order relation with "subset", then from the preceding facts the sets $(\mathcal{D}, \subset)$ and $(d(M) / \sim, \leq)$ are antimorphisms. In this sense, the mapping $\mathcal{U}:[G] \rightarrow d\left(L_{G}\right)$ is antimorphism, and $(\mathcal{D}, \subset)$ is a lattice.

In connection with the former facts, for the function $G \in d(M)$ we define, first, an upper norm, in denoted $\|\cdot\|: d\left(L_{G}\right) \rightarrow \mathbb{R}_{+}^{0}$ by

$$
\|x\|_{(G)}=\inf \left\{a>0: \rho_{G}\left(\frac{x}{a}\right) \leq 1\right\} ;
$$

and, then we can broaden this norm to a limitid upper norm as a function, denoted this extension by $\|\cdot\|_{(G)}^{*}$. In this sense, a hers quasilinear representation is in the following form:

$$
\|x\|_{(G)}^{*}=\sup _{\rho_{G}^{*}(v)<\infty}\left(\frac{\int u\left(v-\psi^{-1}(0)\right) d s}{1-\rho_{(G)}^{*}(v)}\right) .
$$

Theorem 61. If the mapping $T: A \rightarrow A$ satisfy for every $s \in A$ and for every $a \in R$ the following conditions: $|T(a x)|=|a||T x|$ and

$$
\rho_{G^{-1}}(T x) \leq K_{0}+\rho_{R^{-1}}(K x),
$$

where $K>0$ and $K_{0} \geq 0$ are constants, then for every $s \in A$ holds the following inequality in the form

$$
\|x\|_{\left(G^{-1}\right)}^{*} \leq K\left(K_{0}+1\right)\|x\|_{\left(R^{-1}\right)}^{*} .
$$

Proof. Since $T(0)=0$, we have that (100) holds for $\|x\|_{\left(R^{-1}\right)}^{*}=0$. This inequality holds and for $\|x\|_{\left(R^{-1}\right)}^{*}=+\infty$. We set $0<a=\|x\|_{\left(R^{-1}\right)}^{*}<+\infty$. Then we have

$$
\begin{gathered}
\rho_{G^{-1}}\left(\frac{T x}{\left(K_{0}+1\right) K_{a}}\right) \leq \frac{1}{K_{0}+1} \rho_{G^{-1}}\left(\frac{T x}{K a}\right)= \\
=\frac{1}{K_{0}+1} \rho_{G^{-1}}\left(T\left(\frac{x}{K a}\right)\right) \leq \frac{1}{K_{0}+1}\left[K_{0}+\rho_{R^{-1}}\left(\frac{x}{a}\right)\right] \leq 1,
\end{gathered}
$$

whence, from definition of norm $\|\cdot\|_{\left(G^{-1}\right)}^{*}$, we obtain the inequality in the form (100). The proof is complete.

Theorem 62. The fact $G \prec R$ is equivalent with the fact: that there exists a constant $K>0$ such that holds the following inequality in the form

$$
\|x\|_{\left(G^{-1}\right)}^{*} \leq K\|x\|_{\left(R^{-1}\right)}^{*} \quad \text { for every } \quad x \in A .
$$

Proof. If $G \prec R$, then from (99) and Theorem 61 for $T x=x$ we obtain inequality (101). Reversed, let (101) holds. Since $\|x\|_{\left(G^{-1}\right)}^{*}<+\infty$ infty if and only if $x \in d\left(L_{G^{-1}}\right)$, from (101) arise $d\left(L_{R}\right) \subset d\left(L_{G^{-1}}\right)$; and thus $G \prec R$ from a former fact. The proof is complete.

Applying Theorem 62, and from some former facts, directly we obtain the following statements: 
(a) If $G \prec R$, then $d\left(L_{R^{-1}}\right) \subset d\left(L_{G^{-1}}\right)$ and there exists a constant $K>0$ such that holds the following inequality in the form:

$$
\|x\|_{\left(G^{-1}\right)}^{*} \leq K\|x\|_{\left(R^{-1}\right)}^{*} \quad \text { for every } \quad x \in d\left(L_{R^{-1}}\right) .
$$

(b) If $G \sim R$, then $d\left(L_{G^{-1}}\right)=d\left(L_{G^{-1}}\right)$ and there exist constants $K_{0}, K>0$ such that for every $x \in d\left(L_{R^{-1}}\right)$ hold the following inequalities in the form

$$
K_{0}\|x\|_{\left(R^{-1}\right)}^{*} \leq\|x\|_{\left(G^{-1}\right)}^{*} \leq K\|x\|_{\left(R^{-1}\right)}^{*} .
$$

Annotation. We notice that topology on $d\left(L_{\left(R^{-1}\right)}\right)$ is redefined of they which make topology in $d\left(L_{\left(G^{-1}\right)}\right)$. This means that the order relation in the class of $M$-functions have and algebric and topological consequences.

$>$ From inequality $(102)$ arise that the operator $T: d\left(L_{R^{-1}}\right) \rightarrow d\left(L_{G^{-1}}\right)$ defined by $T x=x$ is continuous.

On the other hand, inequality (103) demonstrate that the equivalent $M$-functions determined linear homeomorphic spaces, where the identical mapping is a linear homeomorphism.

In connection with this, we define the following two norms. For an $M$-function $G$ we define the extension limitid upper norm $\|\cdot\|_{G}^{*}: A \rightarrow[0,+\infty]$ by

$$
\|u\|_{G}^{*}=\inf \left\{\int_{u} u\left(v-\psi^{-1}(0)\right) d s:\|v\|_{(G)} \leq 1\right\}
$$

for $u \in A^{+}$and $\|x\|_{G}^{*}=\|\mid x\|_{G}^{*}$ for $x \in A$. On the other hand, for an $M$-function $G$ we define and the extension limited upper norm $\|\cdot\|_{d(G)}^{*}: A \rightarrow[0,+\infty]$ by

$$
\|u\|_{d(G)}^{*}=\inf \left\{\frac{\int u\left(v-\psi^{-1}(0)\right) d s}{1+\rho_{G}(v)}: \rho_{G}(v)<+\infty\right\}
$$

for $a \in A^{*}$ and $\|x\|_{d(G)}^{*}=\|\| x \|_{d(G)}^{*}$ for $x \in A$. For the preceding two norms hold the following inequalities

$$
\|u\|_{d(G)}^{*} \leq\|u\|_{G}^{*} \leq \mathcal{D}\|u\|_{d(G)}^{*} .
$$

From inequalities (104) arise $\|x\|_{G}^{*}<+\infty$ if and only if $x \in d\left(L_{G}\right)$. In the preceding context, for an $M$-function $G$ we define the norm $\|\cdot\|_{G}^{0}: A \rightarrow[0,+\infty]$ by

$$
\|u\|_{G}^{0}=\sup \left\{\int u\left(v-\psi^{-1}(0)\right) d s:\|v\|_{\left(G^{*}\right)} l \frac{1}{2}\right\}
$$

for $u \in A^{+}$and $\|x\|_{G}^{0}=\||x|\|_{G}^{0}$ for $x \in A$. Also, a limited upper norm $\|\cdot\|_{d(G)}^{0}: A \rightarrow$ $[0,+\infty]$ we define by

$$
\|u\|_{d(G)}^{*}=\sup \left\{\frac{\int u\left(v-\psi^{-1}(0)\right) d s}{1-\rho_{G}^{*}}: \rho_{G}^{*}(v)<\frac{1}{2}\right\}
$$

for $x \in A^{+}$and $\|x\|_{d(G)}^{0}=\||x|\|_{d(G)}^{0}$ for $x \in A$. For this two norms hold the following inequalities in the form

$$
\frac{1}{2}\|u\|_{d(G)}^{0} \leq\|u\|_{G}^{0} \leq\|u\|_{d(G)}^{0} .
$$

Annotation. In connection with the preceding spaces we can to speak on ordinary convergence in the sense that $\lim _{n \rightarrow \infty} x_{n}(s)=x(s)$ for $x_{n}, x \in A(n \in \mathbb{N})$; and we can to speak on convergence in middle (of index $G$ ) in the sense that

$$
x_{n} \rightarrow_{G} x \text { if and onli if } \rho_{G}\left(x_{n}-x\right) \rightarrow 0 ;
$$

or we can to speak on convergence in the space $d\left(L_{G}\right)$ as an convergence via norms in a given spaces. 
An inequality. Let $G$ be a $M$-function and we set $p=p(G)$ and $q=q(G)$. Then holds the following inequality

$$
\|u(\lambda s)\|_{(G)} \leq \max \left\{\lambda^{-1 / p}, \lambda^{-1 / q}\right\}\|u(s)\|_{(G)}
$$

for every $u \in d\left(L_{G}([0,+\infty))\right)$ and for arbitrary fixed $0 \leq \lambda<+\infty$. (For the proof of this inequality, from (95) see: Tas ković [2005]).

\section{REFERENCES}

[1] M. R. Tasković: Nonlinear Functional Analysis, (Fundamental Elements of Theory). First Book: Monographs, Zavod za udžbenike i nastavna sredstva, Beograd 1993, 792 p.p., (Serbo-Croation). English summary: Comments only new main results of this book, Vol. 1 (1993), 713-752.

[2] M. R. Tasković: Nonlinear Functional Analysis, Second Book, Monographs - Global Convex Analysis: General convexity, Variational methods and Optimization, Zavod za udžbenike i nastavna sredstva and Vojnoizdavački zavod, Beograd 2001, (In Serbian), 1223 pages.

[3] M. R. Tasković: Theory of transversal point, spaces and forks, Fundamental Elements and Applications, Monographs of a new mathematical theory, VIZ-Beograd 2005, (In Serbian), 1054 pages. English summary: 1001-1022.

Milan R. TASKović

Faculty of Mathematics

P.O. BoX 550

11000 BEOGRAD

Serbia

Home address:

Milan R. TAsković

NeHRuova 236

11070 BELGRADE

SERBIA

E-mail address: andreja@predrag.us 